\title{
Public En-route Traveler Information Connecting Vehicles and Infrastructure: Prototype, Human Factors, and Information Strategies for Dynamic Traffic Management
}

\author{
A Dissertation \\ Presented to \\ The faculty of the School of Engineering and Applied Science \\ University of Virginia
}

\begin{abstract}
In Partial Fulfillment
of the requirements for the Degree

Doctor of Philosophy (Civil and Environmental Engineering)
\end{abstract}

by

Jiaqi Ma

December 2014 



\begin{abstract}
APPROVAL SHEET
This thesis is submitted in partial fulfillment of the requirements for the degree of

Doctor of Philosophy (Civil and Environmental Engineering)
\end{abstract}

Jiaqi Ma

This thesis has been read and approved by the examining Committee:

Brian L. Smith

Michael D. Fontaine

$\begin{array}{r}\hline \text { Brian Park } \\ \hline \text { John S. Miller } \\ \hline \text { William T. Scherer }\end{array}$

Accepted for the School of Engineering and Applied Science:

James H. Aylor

Dean, School of Engineering and

Applied Science

December 2014 


\section{ABSTRACT}

Traveler Information Systems are designed and operated by public transportation agencies to provide travelers with real-time traffic information, enabling them to make better travel decisions. The most commonly used way to provide real-time en-route traveler information to motorists is though Dynamic messages signs (DMS). Despite their effectiveness, they are costly and limited in the amount of information they can deliver. The wide availability of smart mobile devices communication technologies offers the possibility to provide traveler information not only through in-vehicle devices without incurring huge infrastructure costs, but also in a more flexible manner to selected individuals and locations without geographical constraints. To comprehensively develop and evaluate this concept, this research was carried out from three perspectives.

First, this research proposed the concept of VDMS (Virtual Dynamic Message Signs) prototyped a smartphone based application for demonstration and understanding of user experience for future deployment. The user survey reveals a positive attitude among participants toward VDMS in terms of both usefulness and satisfaction, with an average rating of -0.90 and 0.81 on a -2 to 2 (Totally agree to Total disagree) five point Likert scale. The survey also indicates that potentially most drivers $(80.95 \%)$ perceive that VDMS is a safer way to receive information and most drivers $(66.67 \%)$ feel more comfortable receiving information from VDMS compared with DMS. The results indicate great user acceptability and potentials for such systems to be deployed by public agencies in the future.

Second, since the concept of VDMS uses in-vehicle devices, particularly relying on auditory messages to deliver DMS-type messages, this research aims to address the question whether this new way of information delivery conveys information as least as effectively as existing DMSs. A mixed repeated measure experiment was designed using a driver simulator to 
examine the impacts of driver age, information transmission mode, amount of information, and driving complexity on message comprehension, distraction and perceived difficulty. Forty-two Participants were recruited and each of them was tested under different combinations of the three within-subject factors. Participant performance was measured in terms of message comprehension, distraction, and self-reported message difficulty level. Results reveal that VDMS generally performs better than DMS across different amounts of information and under different driving conditions, regardless of driver age. VDMS is significantly better than DMS in message comprehension under relatively complex conditions, reduces reaction time to unexpected stimuli (as measured with a reduced time-to-brake of 0.39 seconds), and makes the same messages easier to process and retain for drivers than DMS. Based on these results, it is recommended that transportation agencies give careful consideration to VDMS as a future strategy for delivering public traffic information in a connected vehicle environment.

Finally, with VDMS as a new powerful tool for public en-route traveler information provision, the study selected, among its many potential applications, routing advisory for emergency traffic management as an example, developed and evaluated corresponding Personalized System Optimum Information (PSOI) strategy, to demonstrate potential benefits of advanced management strategies enabled by VDMS. An efficient queue-based heuristic algorithm was developed for generating VDMS-PSOI routing advisories, in particular considering mixed information use in the traffic network to accommodate the adaptive behavior of other users in the network. Case studies indicated that heuristic algorithm was effective, and existence of PSOI can help shorten average travel times for all types of travelers, with maximum benefits obtained after a certain market penetration (75\% in our case studies). Also, PSOI advisories were not only effective in reducing average system travel time, but also guarantee relatively short personal travel time even compared with user-oriented information groups. Thus, PSOI is recommended by this paper as an advantageous way for next generation advanced 
information systems enabled by VDMS, particularly for emergency traffic management by Traffic Management Centers. 


\section{CONTENTS}

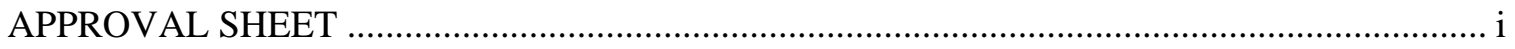

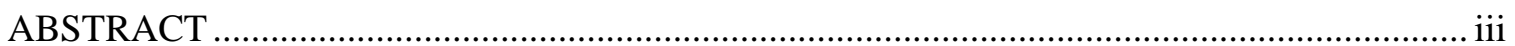

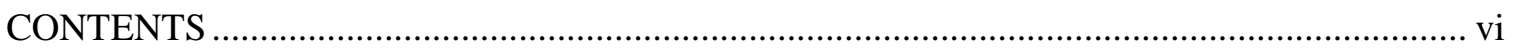

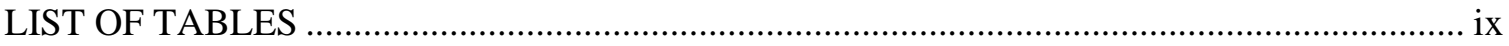

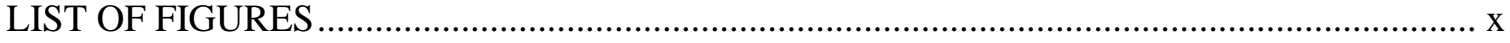

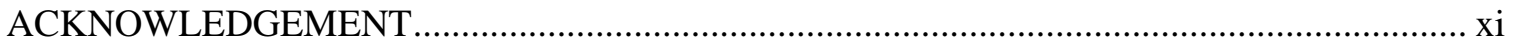

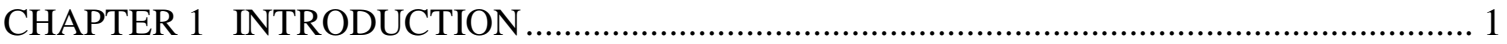

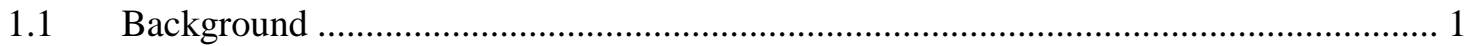

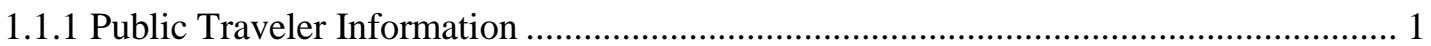

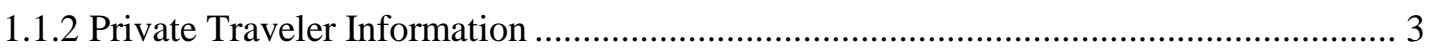

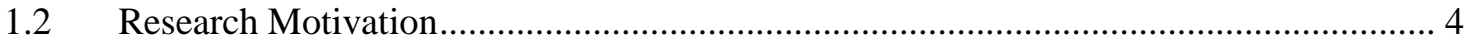

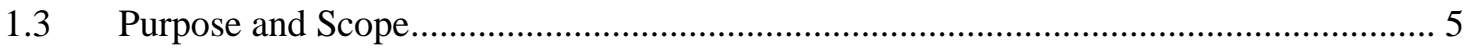

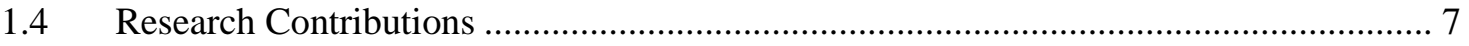

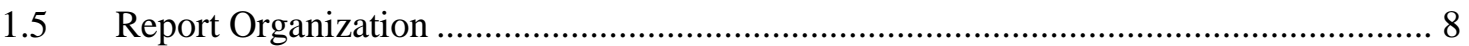

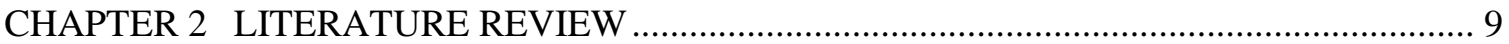

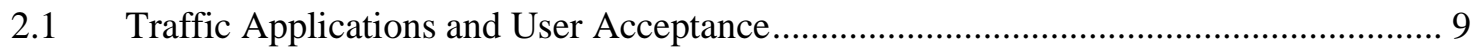

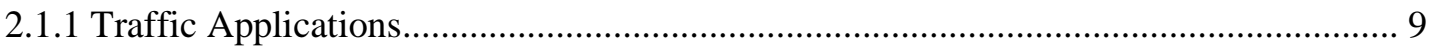

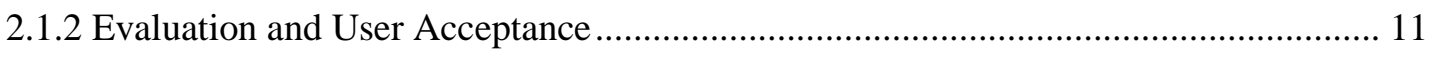

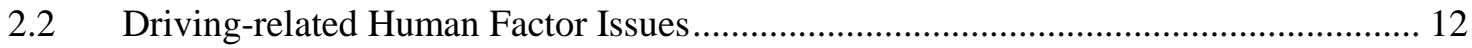

2.3 Dynamic Traffic Management with Real-time Information and Routing .................... 15

2.3.1 Types of Traveler Information Systems for Traffic Management................................ 15

2.3.2 Modeling of traveler information provision for traffic management .................... 16

2.3.3 Mesoscopic Dynamic Traffic Modeling and Simulation.............................................. 19

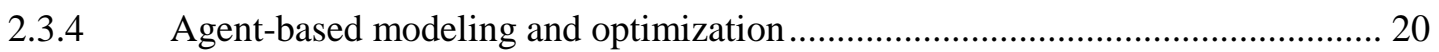

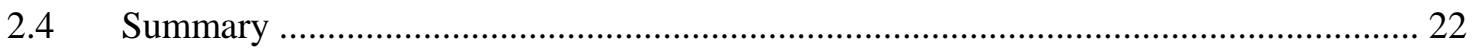

CHAPTER 3 VDMS CONCEPTS, PROTOTYPE AND USER EXPERIENCE ....................... 23

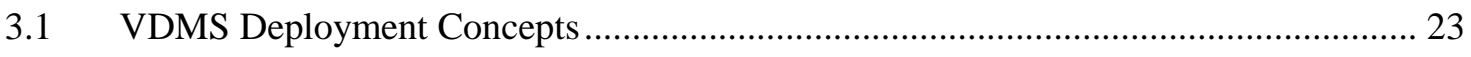

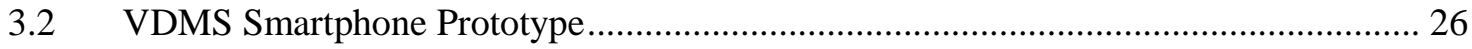

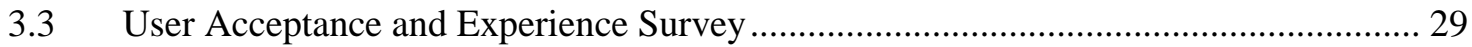


CHAPTER 4 HUMAN FACTORS: EFFECTIVENESS OF IN-VEHICLE AUDITORY MESSAGES 38

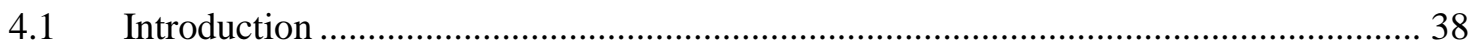

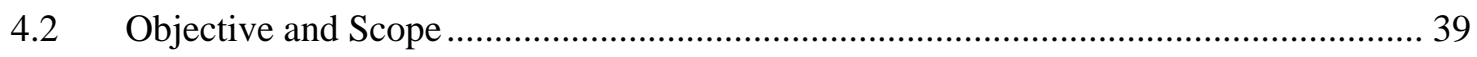

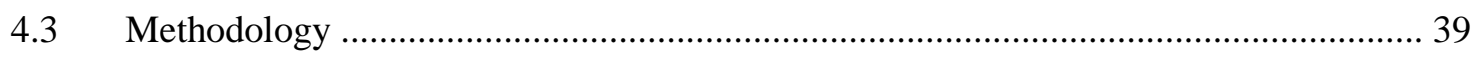

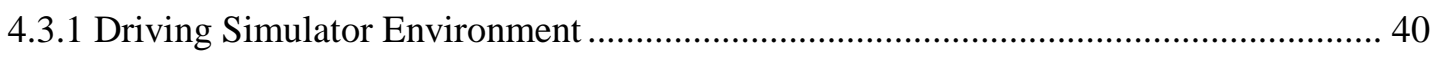

4.3.2 Test Factors and Experimental Design .................................................................. 42

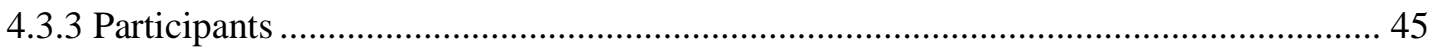

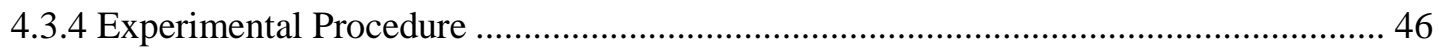

4.3.5 Evaluation Metrics and Data Analysis ....................................................................... 47

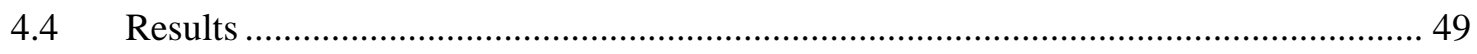

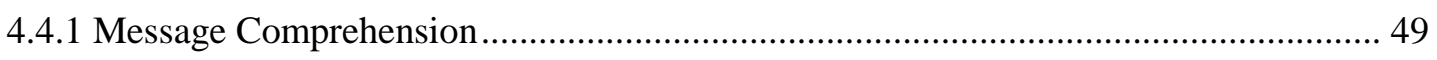

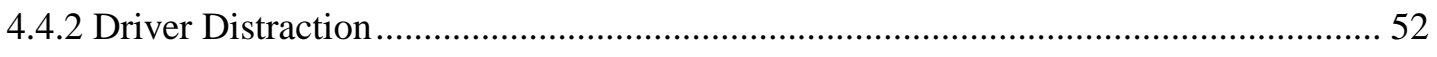

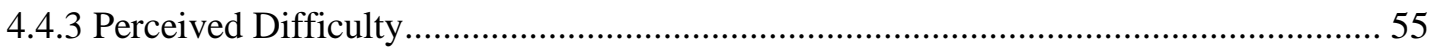

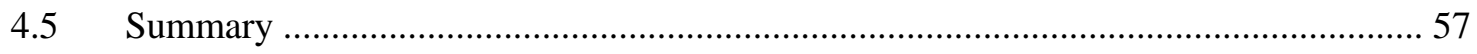

CHAPTER 5 AN APPLICATION: ROUTING ADVISOTIES FOR EMERGENCY TRAFFIC

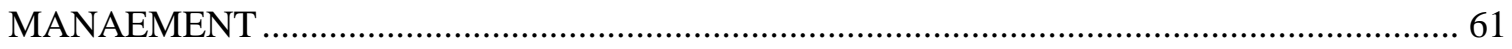

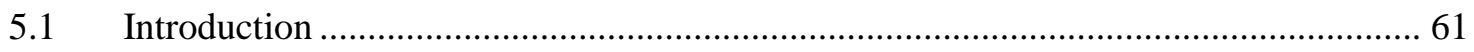

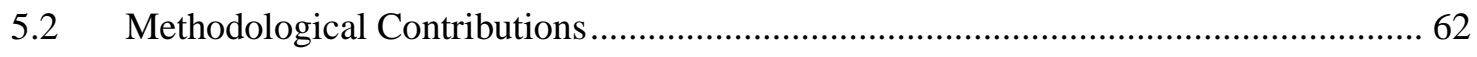

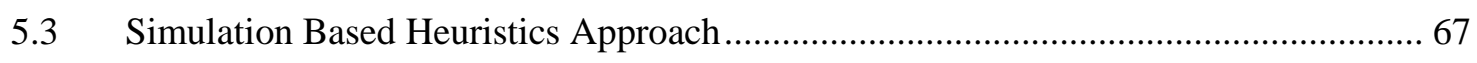

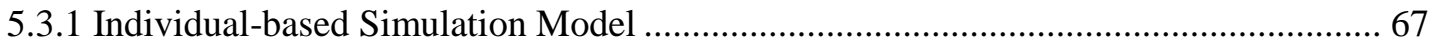

5.3.2 Personalized System Optimum Traveler Information (PSOI) ....................................... 68

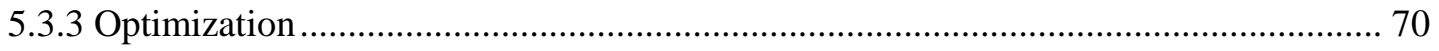

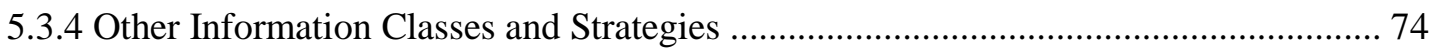

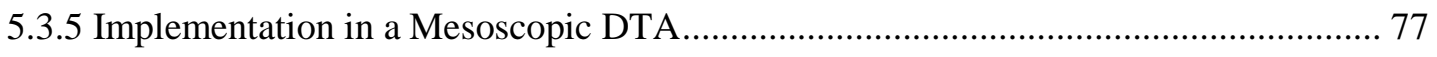

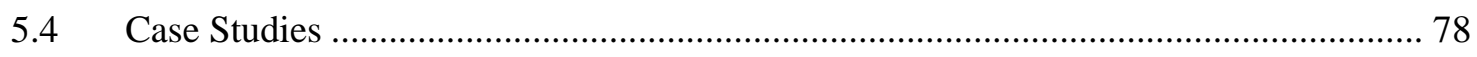

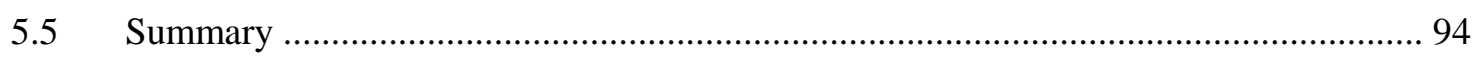

CHAPTER 6 CONTRIBUTIONS AND FUTURE RESEARCH ......................................... 95

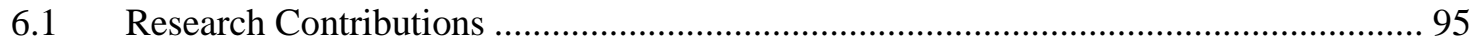

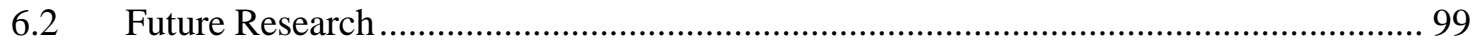

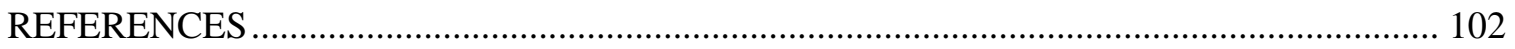

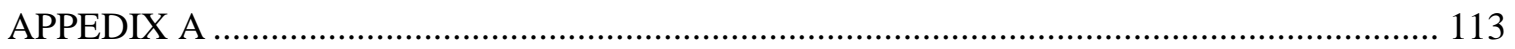

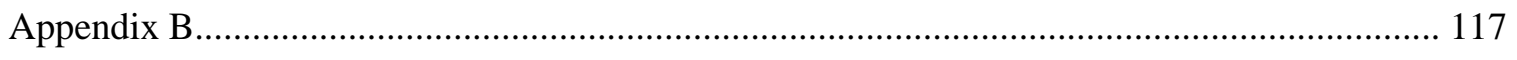




\section{LIST OF TABLES}

Table 2-1 Lists of Existing Well-known Traffic Applications..................................................... 10

Table 2-2 Comparison of Optimization Models, Agent-based Approaches and Real World Systems

Table 3-1Aggregate Results of Nine-item Questions on User Acceptance Level........................ 30

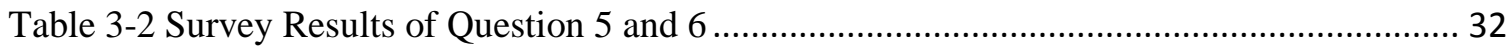

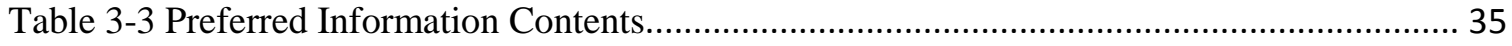

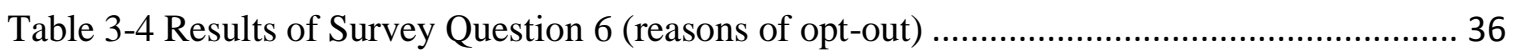

Table 4-1 Analysis Results for Message Comprehension ........................................................... 49

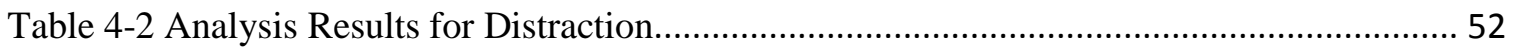

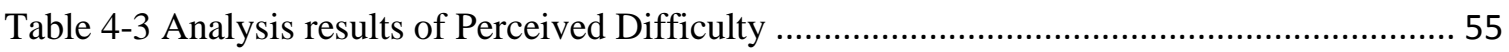

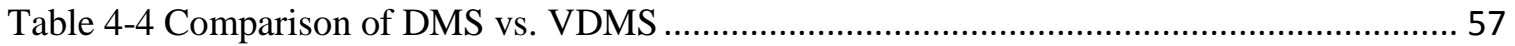

Table 5-5 MOEs of PSOI Simulation Results under Different Demand Levels and Market

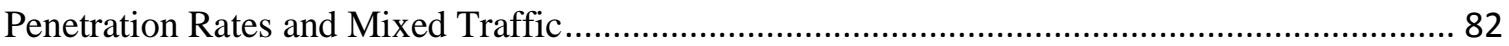




\section{LIST OF FIGURES}

Figure 1-1 Illustration of the Virtual Information Zone ............................................................. 5

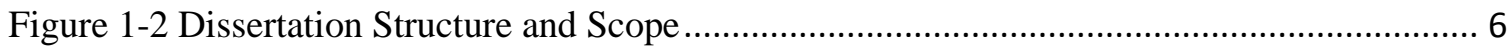

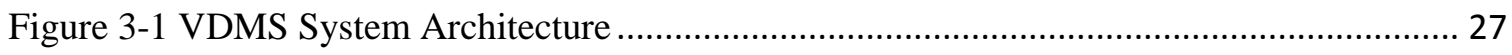

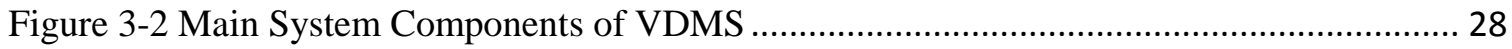

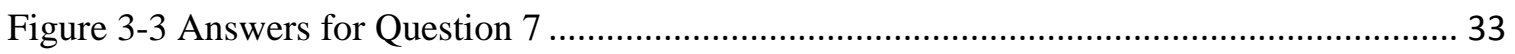

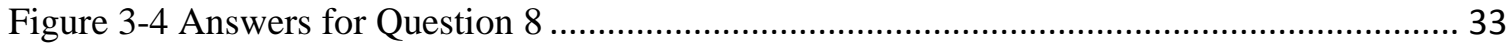

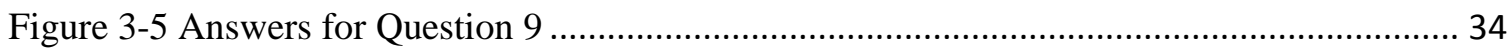

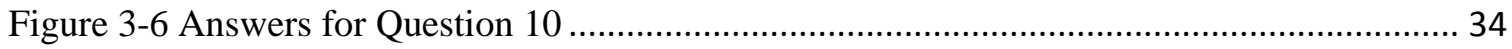

Figure 4-1 Driver Guidance System (DGS, https://www.generalsimulation.com) ...................... 42

Figure 5-1 Comparison between Flow-based Modeling and Individual-based Modeling ............ 64

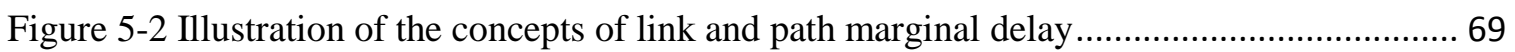

Figure 5-4 Algorithm Performance on Three-corridor Test Network......................................... 80

Figure 5-5 Sensitivity Analysis of Demand Levels and Market Penetration Rates. .................... 87

Figure 5-6 Algorithm Performance of Mixed Traffic Optimization for Three Corridor Network 88

Figure 5-7 Salt Lake City Network and Algorithm Performance ............................................. 90

Figure 5-8 Sensitivity Analysis of PSOI Market Penetration Rate for SLC Subarea Network..... 91

Figure 5-9 Algorithm Performance of Mixed Traffic Optimization for SLC Subarea Network ... 92

Figure 5-10 Sensitivity Analysis of PSOI in Mixed Traffic for SLC Subarea Network ............... 93 


\section{ACKNOWLEDGEMENT}

Sincere thanks to my advisor, Dr. Brian Smith, whose encouragement and guidance in the past years has been invaluable. The service of my dissertation committee is greatly appreciated: Dr. Brian Park, Dr. Michael Fontaine, Dr. John Miller, and Dr. William Scherer. In particular, I am very grateful for all the help that Dr. Fontaine rendered to me in the experimental design and data analysis of the driving simulator human factor study.

This project would not have been possible without the funding from CVI-UTC, Connected Vehicle Infrastructure University Transportation Center. Additional funding from the Virginia Center for Transportation Innovation and Research, a partnership between the Virginia DOT and the University of Virginia, was critical to the research effort.

Many other researchers provided help and suggestions during the preparation of this report. Many thanks to Dr. Xuesong Zhou (Arizona State University) for his help in implementing my solution algorithm in his open-source DTA simulation tool. I also appreciate help from Dr. Michael Demetsky, Dr. Hyungjun Park, and Dr. Emily Parkany.

Thank you to my parents for their unconditional support.

Finally, thank you to my wife for her selflessness and patience. 


\section{CHAPTER 1 INTRODUCTION}

\subsection{Background}

The purpose of Traveler Information Systems is to provide travelers with real-time traffic information, enabling them to make better travel decisions. Relevant information may include locations of incidents, work zones, weather, road conditions, and lane closures, etc. There are many different types of traveler information. From the perspective of time of provision, there are pre-trip information and en-route information. Also, in terms of information sources, there are public and private information. This dissertation focuses on public en-route traveler information provision.

\subsubsection{Public Traveler Information}

Public Traveler Information refers to information provided by public agencies, such as state DOTs and Traffic Management Centers (TMC). This type of information is provided through different channels, such as Dynamic Message Signs (DMS), 511 website, 511 call, 511 smartphone app, and Highway Advisory Radio (HAR), etc.

Among different channels, DMS have been widely used by state DOTs or TMCs for many years to provide real-time en-route traveler information on freeways and arterials. While DMSs have been proven effective, there remain many limitations. First, the amount of information that can be displayed on DMS during the time period when drivers can see the message clearly is very limited, usually only 8 seconds for three information units on each screen 
phase. Second, the very task of reading a DMS is distracting to the driving task. This can be seen in many locations where monitoring sensors report a reduction in speed due to drivers braking to read DMS messages (Dudek \& Ullman, 2006). Such distraction also forces DOTs to use short messages on DMSs - hampering the ability to better inform travelers. Another key concern is that DMSs are fixed assets. They are expensive and can only inform travelers at the location where they are installed. Finally, DMS is one-for-all, placed roadside or overhead for all the passing traffic. This is partly due to TMCs have no information of each traveler and thus can only provide same information to everyone, no matter they need it or not, thus further increasing distraction to travelers who don't need the information. Future deployments should consider innovative information delivery methods to tackle these problems.

Public sector has established traditions of traffic management, and real-time traveler information is one of the powerful traffic management tools. Currently, public agencies publish basic information on daily basis to inform travelers and let them make better own travel decisions. However, public agencies may need to take a more active role in managing traffic, particularly under special emergency conditions. For example, VDOT defined emergency incidents as an occurrence, human or nature-caused, that requires public agencies'responses: 1) weather related emergencies (e.g., flooding, hurricane), 2) severe incidents, 3) hazardous materials spills (hazmat), and 4) terrorist attacks (http://www.virginiadot.org ). Public agencies need to make best use of capacities of existing facilities via various management approaches, such as providing routing advisories, to respond to ensure public safety and or to maintain or restore traffic movement under emergencies. Currently, only general routing information is provided, such as the closure of certain routes or asking all traffic to use alternatives at the same time. This is partly because of the limitation of current information delivery mode DMS, which is deployed at only a few locations and can only provide same general one-for-all messages for all travelers. 


\subsubsection{Private Traveler Information}

Private Traveler Information is provided by private companies, such as Google, INRIX, TomTom, Waze (Google). This type of information is usually provided through website and apps on personal devices like smartphones. Private Traveler Information has three unique attributes compared with current Public Traveler Information.

- Detailed: More information can be provided through personal devices, using audio, text, and map display. Most of the apps can provide basic traffic information similar to DMSs, though usually based on data from different sources (e.g., user report). Also, personal route guidance is usually provided in these apps based on current travel time. In comparison, DOTs usually don't provide route guidance on daily basis but will potentially provide route advisories (e.g., alternative route number) under emergencies and other special conditions.

- Personalized: Personal devices can understand the attributes of each traveler, such as location, destination, value-of-time, and habitual routes, and thus information can be targeted to each driver. Note that vehicle location and destination are key attributes of travelers in terms of en-route information and they can only be obtained via personal devices and real-time communication with infrastructure. In comparison, current public information mode such as DMS is limited from this perspective and future public information can potentially draw upon this attribute.

- User-oriented: Influenced by private companies' business model, Private Traveler Information/route guidance is usually for the purpose to optimize personal travel experiences, such as choosing paths with shortest time for each user. This is quite different from the goal of public agencies - managing traffic and making best use of existing facilities. 
Although this dissertation focuses on public en-route traveler information, technologies widely used in private sectors, such as personal devices and wireless communication, can be drawn upon and tailored for developing concepts of innovative next generation public enroute information.

\subsection{Research Motivation}

The limitations of current public en-route traveler information mode (DMS) greatly constrained the capability of traffic management using traveler information, as discussed previously: inadequate details of information, distraction to driving tasks, one-for-all message delivery (information irrelevant to many travelers), and high infrastructure cost. Thus, we need a new channel to more effectively deliver such public en-route information. The new channel needs to be able to provide more detailed information, including traditional basic information (e.g., location and time of incident, congestion, work zone, and segment travel time) and enable more advanced traffic management strategies when necessary, such as routing advisories under emergencies, targeting each individual based on their attributes such as current location. Also, the new channel needs to not only be able to increase people's comprehension level of information, but also reduce driver distraction, which in turns allow the provision of more detailed information. Also importantly, the new approach should be cost-effective, without relying on huge infrastructure investment and flexible/scalable for wide deployment.

This study proposes the concept that with the advancement of information and communication technology, instead of letting drivers reading roadside message signs, traffic messages can be delivered to each vehicle through in-vehicle devices, such as smartphones or onboard units of connected vehicles. This new concept is named as Virtual Dynamic Message Sign (VDMS) in this dissertation. In addition to the potential benefits mentioned above, it is also a true "mobile" application and true en-route information type - meant to be used while a driver is 
traveling. This distinguishes it from current 511 applications that explicitly state they are to be used only when the vehicle is not in motion.

One example of the VDMS concept can be demonstrated using Figure 1-1. A traveler's location is monitored using the Global Positioning System of the in-vehicle devices. The device sends location information to the server on a certain interval basis and the server matches the GPS to a real location on the server map. When the traveler is detected to be in range of a pre-defined information zone or link (i.e. represented by latitude/longitudes), as shown in Figure 1-1Error! Reference source not found.Error! Reference source not found., the information associated to this information zone/link is presented to the traveler via an audible message. Additionally at the server end, an application is able to retrieve real time information from certain traveler information providers (e.g., VDOT 511) or has an algorithm embedded in to predict and calculate traveler information with access to real time traffic sensors.

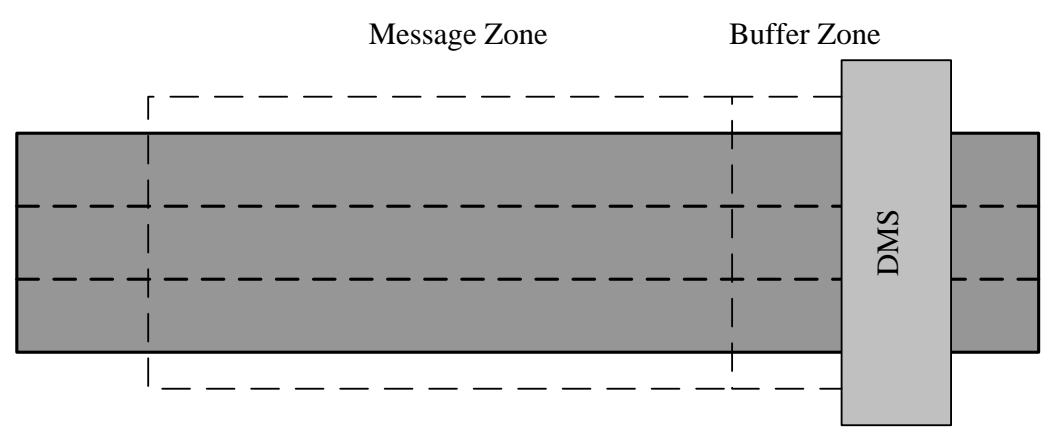

Figure 1-1 Illustration of the Virtual Information Zone

\subsection{Purpose and Scope}

There are several gaps in knowledge that this research addresses to answer two critical questions. The overall structure and scope of this dissertation are demonstrated in Figure 1-2. 


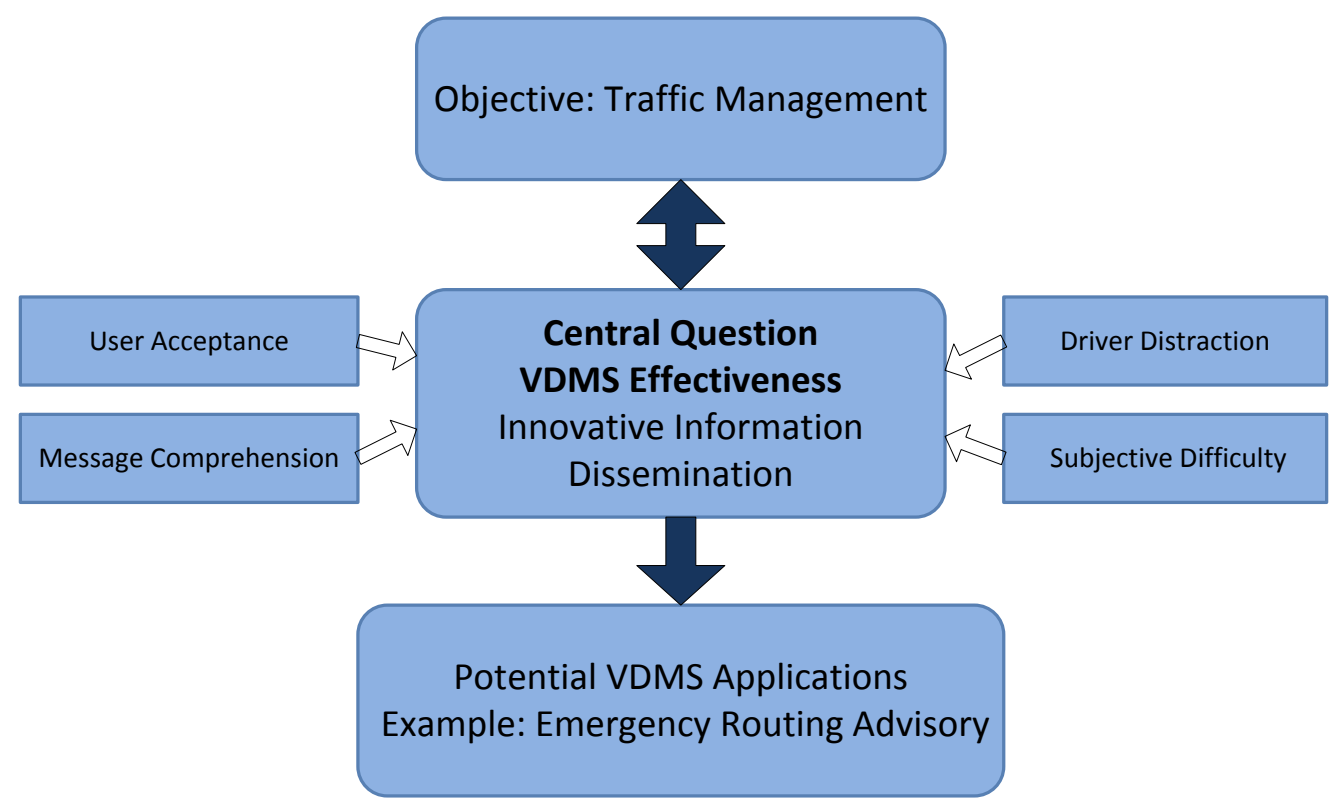

Figure 1-2 Dissertation Structure and Scope

With the objective of improving traffic management, the Central Question is related to the innovative information dissemination method, VDMS. A few sub-questions need to be answered: 1) user acceptance and 2) human factor issues related to driver performance when they receive messages through VDMS. This study first takes steps to prototype this application system, and assesses the acceptance and attitudes of travelers, to understand the possibilities and issues to be addressed for future wide deployment of such systems. Next, since delivering DMS-type traffic messages via audio through in-vehicle devices is completely different from display on DMSs, it is necessary to study the effectiveness of VDMS in communicating messages to travelers, especially in terms of information comprehension and driver distraction. This study will consider current roadside DMSs as baseline and investigate whether VDMS will be a viable way to deliver en-route traffic message to drivers in the future when in-vehicle devices are in place.

After the effectiveness of VDMS is confirmed by answering the Central Question, along with its capability of scalability and personalization, we are confident that VDMS can be a 
powerful tool enabling advanced traffic management strategies, such as emergency routing advisory, lane changing advisory and speed advisory. Among numerous potential applications, the dissertation explores the possible benefits VDMS could provide in terms of traffic management under emergencies by developing a potential routing strategies.

\subsection{Research Contributions}

This research will produce several major contributions to the state-of-knowledge. These contributions include:

1) Introduction of VDMS concepts and evaluation of user acceptance/experience via a prototype application. This is one of the first smartphone based connected vehicle traveler information system concepts and prototypes for public en-route traveler information.

2) Investigation of the effectiveness of VDMS in delivering traffic information from the perspective of message comprehension, distraction, and subjective difficulty level. By conducting a driving simulator based human factor experiment, we obtained deep understanding of VDMS characteristics compared with DMS, and the results can serve as evidence for agencies to deploy such systems in the future.

3) Demonstration of potentials of VDMS applications as a powerful tool for traffic management under emergencies. A Personalized System Optimum Information (PSOI) strategy is proposed for developing emergency routing advisories. A queue-based simulation solution heuristics is developed for efficiently solving real-time for advisory strategies for large networks to enable such strategy's capability in real-time deployment. 


\subsection{Report Organization}

The remaining chapters of this dissertation are organized as follows:

Chapter 2 summarizes the review of the literature. Some of the investigated research topics include current public and private traffic information provision, human factor issues related to traveler information and other driving aspects, and dynamic traffic management using realtime traveler information and routing.

Chapter 3 presents the development of a smartphone-based Virtual Dynamic Messages Signs (VDMS) and a user experience survey to assess the acceptance of such a system. Survey results are analyzed and conclusions are drawn on user acceptance of VDMS.

Chapter 4 describes a human factor experiment to investigate the effectiveness of using in-vehicle auditory in delivering traffic messages compared with current Dynamic Message Signs. The data analysis is conducted and results are discussed.

Chapter 5 describes a VDMS-enabled emergency routing strategy with the development of a PSOI simulation based algorithm. Benefits are evaluated to demonstrate potentials of such strategies.

Chapter 6 summarizes the research efforts and its key findings, and discusses potential research directions in the future. 


\section{CHAPTER 2 LITERATURE REVIEW}

The literature review was conducted to survey past work on issues related to advanced traveler information systems and dynamic traffic management. The literature was sorted into three categories for this section:

- Traffic applications and user acceptance

- Driving-related human factor issues

- Dynamic traffic management with real-time information and routing

\subsection{Traffic Applications and User Acceptance}

\subsubsection{Traffic Applications}

With the advancement of communication and mobile devices, a variety of traffic applications have been developed to improve travel experiences. The connected vehicle technologies have injected new vitality to the development of various ITS applications, such as intelligent cruise control and collision avoidance system. A lot of research has developed and evaluated many connected vehicle applications, either using cellular-based smartphones or DSRC-based on-board devices. Smartphone technology and cellular networks, especially 4G/LTE networks, have been well developed and applied for both collection and dissemination of traveler information since the 1990s. Smartphone technology has been used by many developers and researchers in recent 
transportation application development, such as safety enhancer for bike riders (Jin et al., 2012), assistance application for visually impaired pedestrians at intersection crossovers (Liao, 2013), and green-behavior application (Brazil et al., 2013). Since DSRC technology development is still in its infancy, most of relevant researches are simulation-based system evaluation. For example, Park and Smith (2012) investigated benefits of IntelliDrive in Lane Changing Advisory of Freeway Operations. Lee et al. (2013) assessed sustainability benefits of Cooperative Vehicle Intersection Control at an Urban Corridor. Goodall et al. (2013) made use more enriched data from connected vehicles environment to improve traffic signal control algorithms.

Specifically, this dissertation is interested in traffic applications that provide travelers with traffic information and aid them in travel choices. While there are large numbers of traffic applications in the market, we list a few well-known applications and their corresponding descriptions.

Table 2-1 Lists of Existing Well-known Traffic Applications

\begin{tabular}{|l|l|}
\hline Name & Description \\
\hline $\begin{array}{l}511 \text { website and } \\
\text { app }\end{array}$ & $\begin{array}{l}\text { Provides real time traffic information as well as updates on traffic conditions } \\
\text { and weather forecasts. }\end{array}$ \\
\hline Sigalert & $\begin{array}{l}\text { Provides information on current road conditions, speed information and } \\
\text { access to live cameras to aid commuters }\end{array}$ \\
\hline Waze & $\begin{array}{l}\text { Waze relies on user participation for reporting of traffic, car accidents and the } \\
\text { presence of speed traps. Once a report is made by one user, other users are } \\
\text { able to access that report using the Waze map. }\end{array}$ \\
\hline Beat the Traffic & Beat the Traffic shows real-time maps of road and traffic conditions for users. \\
\hline iTraffic & $\begin{array}{l}\text { Provides live traffic maps, showing traffic speeds and incidents on major } \\
\text { routes for hundreds of cities and suburbs to help you plan your schedule and } \\
\text { route. }\end{array}$ \\
\hline Inrixtraffic & $\begin{array}{l}\text { Provides users with real time traffic information, traffic impacting incident } \\
\text { information, traffic forecasting, reported incidents and projected arrival times } \\
\text { for Windows Phone, iOS and Blackberry users }\end{array}$ \\
\hline view2road & $\begin{array}{l}\text { Allows users to view live cameras of certain road locations using iOS and } \\
\text { Windows Phone devices }\end{array}$ \\
\hline traffic.com & $\begin{array}{l}\text { App gives users information on traffic congestion, incident information, } \\
\text { savable routes and real-time traffic maps }\end{array}$ \\
\hline
\end{tabular}


All applications listed in Table 2-1 can provide real-time traffic information on travel time, incidents, work zone, lane closures and road weather conditions with different data sources. For example, State DOT 511 websites make use of both public detector data and private sector data DOTs purchased from private companies to provide traveler information, while private apps like Waze relies heavily on the report of traffic by users. Programs like Connected Citizens by Waze are enabling private companies have access to traffic data from public side and thus provide their users with more comprehensive data.

However, these apps, both public and private, are mostly supposed to be used when the vehicle is not in motion, since the level of driver involvement in using the app is high and using them en-route would cause great safety concerns.

\subsubsection{Evaluation and User Acceptance}

Currently, most of the research only studied the system effectiveness after deployment either in simulation or prototypes. Whereas excellent system performance may be sufficient for the technician, it is as important that the equipment is appealing for and accepted by the driver. For many advisory systems, the main issues determining the feasibility are not of a technical nature, but concern the social context for introduction (Rothengatter, 1991). A prerequisite for the introduction of new in-vehicle technology is acceptance by the public. It is unproductive to invest effort in designing and building an intelligent "co-driver" if the system is never switched on, or even disabled.

There is no standard way of measuring driver acceptance of new technology. A review of the literature shows that there are almost as many methods of assessment of acceptance as there are acceptance studies and they are not much development of evaluation methodology. Systems are evaluated on their pleasantness and usefulness (Kuiken \& Groeger, 1993), the comfort and benefit (Becker et al., 1995) or the ease or degree of use (Crosby et al., 1993). Highlighted 
aspects in the evaluation of the provided information are the content or the format, the reliability, the relevance, the accuracy or the effectiveness of the information (Crosby et al., 1993; Schafer et al., 1993). Acceptance sometimes includes the intention to purchase the system and assessment of the price people are willing to pay (e.g., Becher, 1995). Van Der Laan (1997) proposed a set of nine questions specifically to assess the acceptance level from the perspective of the usefulness and satisfaction. Statistical methods were also proposed for the Likert scale data obtain from the 9 questions.

\subsection{Driving-related Human Factor Issues}

Human factors in the driving environment, including scenarios with traveler information, have been studied extensively in the past several decades. Dudek (1970) did a comprehensive review of studies for various real-time traffic message modes before 1970s, including external visual message (DMS and static signs), in-vehicle visual and auditory (radio). However, this study and earlier ones only made use of simple evaluations such as preference survey and proposed more questions, especially on human factors, to be answered to guide future public and private message design.

Since then, due to the wide use of Dynamic Message Signs (DMS), many researchers have conducted studies on related human factor issues, aiming to understand best practices of message and display design for better driver comprehension and less distraction. Most of these famous studies were conducted by Dudek and his associates (Dudek, 1980-2006), investigating various aspects of DMS information such as information quantity, font size, flashing lines, abbreviation use and consecutive portable DMSs, through the use of either lap top or real car based driving simulator. The quality of the information delivery was measured by the drivers' correct recalling of messages, reaction time and stated preferences. Some other research makes 
use of real traffic counts data or survey data to study the effectiveness of various message display mechanisms. For example, Peeta et al. (2000) found different response rate (measured by willingness to divert) for different types of information using a roadside stated preference survey. The results showed that travelers are willing to divert when detailed information are given. Schroeder and Demetsky (2011) used detector data from Richmond, Virginia, to estimate diversion rates attributable to different DMS advisory messages and the results showed trends where the usage of particular words in messages is more effective than others in causing diversion.

In terms of in-vehicle systems, most of the literature focused on in-vehicle route guidance systems, which are usually used by drivers to optimize personal trips. Literature has been found on various aspects of guidance systems, including audio message content, visual message display and content, digitized versus synthesized speech, timing of information, location of visual displays, and driver interaction with information devices. Walker et al (1990) argued that an audio message should not be very long and should provide only the information necessary for route following. Schraagen (1993) suggested that direction of next turn and distance to turn is most essential and street names and landmarks should not be provided since street name signs are sometimes difficult to locate or are completely absent from the road. Roelofs (1987) and Srinivasan (1995) indicated overwhelmingly preferred recorded speech over synthesized speech, although tracking performance, response times, and errors did not indicate any differences between recorded and synthesized speech.

There were also many studies on the comparison between in-vehicle visual and audio route guidance system. They usually compare following types of information: audio, visual text (symbols, font size, color [Campbell et al., 1997]), visual maps (orientation [Burnett \& Joyner, 1997], format for turn-by-turn displays [Srinivasan et al., 1998; Jonathan, 2010]), and the combination of either two or three approaches [Parkes, 1993; Fastenmeier, 1994]. Most of these 
studies agreed on the conclusion that audio system lead to less distraction from the driving task and is preferred when the information is simple and short and especially when the visual system of the person is overburdened. Studies in the recent decade focused on the human factor issues with advanced technologies, such as driver interaction with guidance systems such as touch screens and smartphones (Kelly, 2005), and in-vehicle warning systems under connected vehicles environment (Bousquet \& Peck, 2008).

Other studies on human factors issues have also enriched methodology and our understanding of human performance under various conditions of driving, such as the use of cell phone (Brookhuis, 1991;Horrey \&Wickens, 2004), listening to music (Unal, 2013), and existence of multi-media devices in the car (Hatfield J. \& Chamberlain, 2008).

An additional critical aspect is the relations of mental arousal and driver performance. The effect of driving conditions, simple or complex, has attracted great attention as well. Research suggests that the existence of secondary task will not necessarily deteriorate driving performance, usually a measure for distraction level, and sometimes perform even better under simple driving conditions (i.e., monotonous driving) (Atchley \& Chan, 2011; Unal et al., 2013). This argument is in line with the Yerkes-Dodson law (Teigen, 1994), which posits that the relationship between task performance and arousal can be depicted by an inverted U-shaped curve. When one's arousal level is too high or too low, performance is predicted to be inhibited, while a moderate arousal level is expected to result in higher performance. Interestingly, for monotonous tasks, an increase in mental effort might be expected when the arousal level is below ideal and when the performer is deactivated due to feelings of fatigue or boredom due to the socalled state-related effort such as fighting boredom or fatigue (De Waard, 1996; Hancock \& Verwey, 1997). These findings are especially important to our study, since reading and comprehending messages are needed under both simple and complex driving conditions, and it is necessary to quantify driver performance on both tasks under both conditions. 


\subsection{Dynamic Traffic Management with Real-time Information and Routing}

\subsubsection{Types of Traveler Information Systems for Traffic Management}

Conceptually, a traveler information provision service presents a driver with information about potential routes to their destination (e.g., travel time, delay, incident location, etc.), and the driver can select a route based on that information. This information can be provided before the trip is made (pre-trip), perhaps through a 511 telephone service or a website like Google Maps (https://www.google.com/maps) or a DOT information (511) website (e.g., http://www.511virginia.org/). Information can also be provided during travel (en-route), which can be accomplished through GPS and mobile phone applications, or variable message signs (VMS). The information could describe congestion levels (indicated by different colors and types of lines on a map), incident locations or detailed travel time and diversion routes.

Vehicle routing advisories, as a special type of traveler information most provided by private sectors, could be either based on instantaneous traffic state when users make the requests or of predictive nature with embedded algorithm to estimate actual travel time the travelers need to expect to complete the specific trip. The routing could be either User-Optimal (UO) or System Optimum (SO)-based. UO information provides best routes to users to minimize their own trip time while SO information offers routing advisories that will optimize the whole system performance. Note that UO information usually cannot guarantee best system performance and are provided by services of private sectors. Meanwhile, SO information is conceptually preferred by public agencies to manage real time traffic and relieve traffic congestion (Paz et al, 2006; Ziliaskopoulos, 2000).

Additionally, most types of current traveler information are one-for-all, such as roadside Variable Message Signs (VMSs), Highway Advisory Radio (HAR), and information websites, 
which provide same information to all travelers/users. Meanwhile, the advancement of personal and in-vehicle devices makes personalized information more feasible (e.g., http://www.inrixtraffic.com/; https://www.waze.com/). This provides users with information specific to each user's characteristics, such as origins, destinations, habitual routes, value of time, and allowable budgets for congestion tolling. Reducing irrelevant en-route information that travelers need to process can greatly reduce driver distractions on the road.

\subsubsection{Modeling of traveler information provision for traffic management}

As the most common approach of traffic management, the use of VMS to manage real-time traffic has been studied by many researchers from different aspects. Peeta et al. (2000) conducted a stated preference survey along a freeway corridor and examined various driver diversion behavior with different VMS messages contents, including location of accident, expected delay, detour strategy and various combinations of the above. Their study shows that the maximum of willingness to divert occurs when all three information contents are provided. A later study (Peeta et al., 2001) incorporated the driver response in a three-step VMS control heuristic, including activation, message display and update. The required diversion rate is determined by the different system-optimal assignment proportions (prepared offline using mean OD demand) and multiple user class situations (no-information user and real-time information user). Xu et al (2012) incorporated an aggregated driver behavior model with attributes that can be obtained online (traffic message, traffic flow, weather and incident). The behavior model is calibrated on-line and was used in a feedback control to decide optimal traffic information. These studies focused on "optimal" VMS contents considering entire passing vehicles as a whole, while not explicitly considering which drivers or what proportion of them need to or should receive real time information. 
The VMS location problem is closely related to the information provision problem in this paper. Since VMS is a fixed asset requiring a large amount of capital investment, most of the studies were to determine where to install the VMS so that the long-term system performance was optimal. A study by Abbas and McCoy, 1999 sought to determine the locations for VMS to maximize potential benefits, defined as the sum of changes in delay and accidents on the freeway upstream and downstream of the incident and those on the alternative routes. The reduction in delay as a result of diversion was computed by the demand-capacity analysis procedure in the Highway Capacity Manual. Chiu et al (2007) extended the analysis to a real-world network level, aiming to minimize the expected network travel time. It was accomplished by finding the VMS locations that yield the least network travel time over many possible incident realizations (that follow a known probability distribution function). However, most of the current information location studies are at planning stage for fixed assets for long term benefits, not for real-time traffic operations. Of particular relevance to this study is that Huynh (2003) addressed the problem of finding the best locations for portable VMS to divert traffic when incident occurs so that the impact on system performance of incident is minimized using a Greedy-Drop heuristic algorithm. However, due to limited on-line searching time, this method could only provide nearoptimal solution, and consider a maximum of 3 portable VMSs on limited number of candidate links due to the exponentially increasing amount of combinations. The information content in these studies is usually updated travel time for some of or all network links. Also, another simplification in these studies is that information provision time is mostly pre-specified as congestion/incident duration with a small time lag considering congestion/incident detection. This simplification may lead to sub-optimal information strategy since there may be available capacity on incident links at the start or during the incident at the incident link. Displaying information until incident clearance may underutilize incident link capacity and cause excess diversions. For similar considerations, information does not necessarily need to be provided right away so that network capacity could be fully utilized. Additionally, traditional message provision rarely 
provide information on alternatives, which is less favorable to travelers and makes the network less controllable since we cannot know what alternatives people will use in response to the simple information.

In the broad field of real-time traffic management modeling there are multiple subcategories that focusing on vehicular flow management, for example simulation-based traffic assignment models, reactive feedback control, mathematical programming and dynamic programming. A dynamic programming approach (e.g., Charbonnier,1991) produces optimal solutions and many insights because of its analytical nature, while it can only applies to small size problems due to the huge computational burden associated with the large number of required modeling states in the formulation. The simulation-based approach can better describe complex traffic flow dynamics required for real-world traffic flow control applications. A number of studies along this line (Mahmassani \& Jayakrishnan, 1999; Paz \& Peeta, 2007; Bolellia, 1991; Hawas \& Mahmassani, 1995; Hawas, 2012) strike to seamlessly integrate DTA simulators a descriptive component into general optimization or feedback control frameworks. The mathematical programming approach has received continuous attention from the research community, as its analytical nature offers many insights into the problem. Various optimization models have been proposed in the literature to produce simple and compact formulations, such as linear programming, so that the resulting problems are easy to solve by standard optimization solvers. On the other hand, dynamic network structures introduced by DTA optimization models are still quite complex and most of the existing models targets only simple test networks, such as single destination cell transition model based formulation by Ziliaskopoulos (2000). It should be also remarked that, most of the optimization models in the literature are based on time-dependent origin-destination flow, with a typical departure time interval of 5 or $15 \mathrm{~min}$. This flow-based modeling paradigm is adequate for finding dynamic system optimal through flow-based traffic management strategies such as signal controls or aggregated information provision. However, it is 
difficult for these models to flexibly model the attributes and behavior of each individual traveler. In contrast, the PSOI represents a fundamentally new approach for real-time ATIS system modeling under ubiquitous communication, which allows traffic system operators to fully optimize and coordinate individuals' trip plans according to the personal value of time, allowable budgets for congestion tolling, and willingness to taking detours.

\subsubsection{Mesoscopic Dynamic Traffic Modeling and Simulation}

Traffic modeling and simulation has long been used for analysis of traffic network and operational strategies evaluation. The models and simulation tools can be categorized as macroscopic, mesoscopic and microscopic models. Macroscopic models can be applied to regional large network at the cost of modeling granularity. On the contrary, microscopic models describe each vehicle/driver's behavior in details at the cost of computational complexity. Thus, they are usually applied to small-scale problems. Among the three types of models, mesoscopic models (Dynamic Traffic Assignment, DTA) are considered as the appropriate modeling granularity for applications of network level traffic management strategies, which may lead to changes of travel choices. These models are usually able to track individual veicles, but focusing more on network flow propagation behavior instead of micro car following behavior. DTA models are able to address larger transportation networks than microscopic simulation but also simulate individual vehicles' movement and choice dynamically during each vehicle's trip.

Multiple mesoscopic models have been developed in the literature in past decades. Vickery (1963) adopts concepts of bottleneck model for dynamic network flow propagation. In this model, agents always move along a link with the free flow speed until they arrive at the exit point, where they form a queue if the outflow rate (or inflow rate of next link) they induce exceeds the maximum value (capacity flow) of the link. Since the bottleneck model ignores the 
physical length of agents and assumes that a queue occupies a point, it is also known as the vertical queue or point queue (P-Q) model. Other dynamic network loading models can also be adopted in the optimization framework we proposed here with some modification, such Newell's simplified kinetic wave (Newell,1993; Lu,2013) and cell transmission model

(Daganzo,1994,1995). However, using more complex models may add to complexity of the optimization framework and increase computation burdens.

There are many simulation-based DTA tools available for this study purpose, such as DYNASMART (Jayakrishnan et al., 1994), DTALite (Zhou \& Taylor, 2014), DynaMIT (BenAkiva et al., 2012) and DynusT (Chiu et al., 2008). These tools all claim to be agent-based, meaning they can track each individual vehicle. However, most of current of their applications are still flow-based models and the internal algorithm coded in these tools are still flow based (e.g., Lu, 2008 \& 2009). Among all the tools, the open-source DTALite is selected for a quick demonstration using a data hub to bridge the model and algorithm with many data sources. Many studies have successfully used it as the engine for prediction and evaluation (Yang \& Zhou, 2014; Jia et al., 2011).

\subsubsection{Agent-based modeling and optimization}

The agent-based modeling approach has received increasing attention by transportation researchers to capture personal characteristics in traveler's daily activities, such as route choice, departure time choice, en-route diversion, as well as the interactions between individual agents. An early research by Dia (2002) models driver's route choice behavior based on real-time traffic information provided by dynamically changing road signs using an agent-based simulation approach, Adler and Blue (2002) suggested a solution concept for the efficient usage of network capacity with the following agents: network managers, information service providers, and 
individual drivers negotiating for network resources. Zhang and Levinson (2005) developed an agent-based travel demand model in which travel demand emerges from the interactions of three types of agents in the transportation system: node, arc, and traveler. Simple local rules were efficiently used to solve complicated transportation problems, such as traffic distribution and assignment. Zhang et al. (2008) explored the welfare consequences of product differentiation using heterogeneous user agents on a congested mixed ownership network. Zhu et al. (2008) proposed an Agent-based Route Choice (ARC) model to track choices of each individual decision-maker in a road network over time and map individual choices into a macroscopic flow pattern.

While the agent-based approach shows its effectiveness in capturing individual behavior and system dynamics, its use in system-wide traffic management and optimization is still limited by several intrinsically challenging modeling characteristics. Agents are commonly modeled through rule-based mechanism and in a spatially distributed manner, which makes it difficult to construct a global traffic state representation and thus difficult to guide agents' behavioral choice to reach a global optimal solutions. In order to optimize agent-based systems, researchers proposed various combinations of agent-based models with mathematical optimization tools, to name a few, Persson et al., 2005; Davidsson et al., 2007; Gaffuri, 2006; Gjerdrum et al., 2001. In the specific area of transportation control and management, Chen et al. (2014) recently introduced a simulation based optimization approach to improve the system-wide performance represented by an agent-based DTA simulator. Table 2-2 shows the comparison of elements of optimization models, agent-based approaches and actual requirements of effective dynamic traffic management strategies.

Table 2-2 Comparison of Optimization Models, Agent-based Approaches and Real World Systems

\begin{tabular}{|l|l|l|l|}
\hline Attributes & Optimization approach & Agent-based approach & $\begin{array}{l}\text { Traffic management } \\
\text { requirements }\end{array}$ \\
\hline Problem size & $\begin{array}{l}\text { Able to solve small to } \\
\text { Medium sized problems }\end{array}$ & $\begin{array}{l}\text { Scalable since only } \\
\text { local behavior is }\end{array}$ & $\begin{array}{l}\text { Small to large size, depending } \\
\text { on problem nature and network }\end{array}$ \\
\hline
\end{tabular}




\begin{tabular}{|l|l|l|l|}
\hline Decision level & $\begin{array}{l}\text { Centralized decisions } \\
\text { made for all travelers }\end{array}$ & $\begin{array}{l}\text { Donsidered } \\
\text { Distributed decisions } \\
\text { made by each traveler }\end{array}$ & $\begin{array}{l}\text { topology } \\
\text { Centralized decisions should be } \\
\text { made, while considering vehicle } \\
\text { characteristics, behavior and } \\
\text { response }\end{array}$ \\
\hline Resolution level & $\begin{array}{l}\text { Global view with } \\
\text { simplified system } \\
\text { description without } \\
\text { considering detailed } \\
\text { traveler characteristics }\end{array}$ & $\begin{array}{l}\text { Detailed description of } \\
\text { characteristics and } \\
\text { behavior of different } \\
\text { agents, but lack of } \\
\text { global view }\end{array}$ & $\begin{array}{l}\text { Need traveler details } \\
\text { consideration but also need to } \\
\text { trade it off for control ability } \\
\text { due to incurred computational } \\
\text { burden }\end{array}$ \\
\hline Communication & $\begin{array}{l}\text { Low communication } \\
\text { requirement, only } \\
\text { needed once between } \\
\text { controller and travelers }\end{array}$ & $\begin{array}{l}\text { High communication } \\
\text { load, needed frequently } \\
\text { between agents }\end{array}$ & $\begin{array}{l}\text { Mutual communication needed } \\
\text { between controller and } \\
\text { travelers. }\end{array}$ \\
\hline Solution quality & $\begin{array}{l}\text { Solution of global } \\
\text { optimum; consider final } \\
\text { realized system state } \\
\text { (e.g., route travel time) } \\
\text { with predicative ability }\end{array}$ & $\begin{array}{l}\text { No guarantee of system } \\
\text { optimum since only } \\
\text { local rules considered; } \\
\text { only consider current } \\
\text { system state for each } \\
\text { step without predicative } \\
\text { ability }\end{array}$ & $\begin{array}{l}\text { Need system optimum solution } \\
\text { and also consider individual } \\
\text { characteristics and preferences; } \\
\text { Need to be predicative to } \\
\text { consider realized system state } \\
\text { for better strategy provision }\end{array}$ \\
\hline
\end{tabular}

\section{$2.4 \quad$ Summary}

This chapter reviewed some of the important research in recent developments related to advanced traveler information systems and other connected vehicle applications. A brief overview of connected vehicle applications was provided. Issues of human factors related to advanced traveler information systems were reviewed. Also, recent developments in analytic and simulation-based models regarding dynamic traffic management, particularly traveler information provision and routing, were surveyed and solution algorithms to the models were also reviewed. 


\section{CHAPTER 3 VDMS CONCEPTS, PROTOTYPE AND USER EXPERIENCE}

\subsection{VDMS Deployment Concepts}

Transportation agencies have a unique and important role in providing traveler information to enable the safest and most efficient overall utilization of existing transportation facilities. Considering the limitations of current public en-route traveler information mode (DMS), we need a new channel to more effectively deliver such en-route information. The new channel needs to be able to provide more detailed information, including traditional basic information (e.g., location and time of incident, congestion, work zone, and segment travel time) and enable more advanced traffic management strategies when necessary, such as routing advisories under emergencies, targeting each individual based on their attributes such as current location. Also, the new channel needs to not only be able to increase people's comprehension level of information, but also reduce driver distraction, which in turns allow the provision of more detailed information.

The concept of Virtual Dynamic Message Signs (VDMS) is proposed in Chapter 1 to meet these needs and overcome limitations of current DMSs. This section firstly discusses in detail a few functionalities that are critical to the success of next generation traveler information systems enabled by VDMS concept.

(1) Auditory message 
To avoid the problems of DMS that messages are line-of-sight dependent and require user efforts in reading the messages, we propose to replace the simple roadside text message with a detailed in-vehicle audio message. First, the audio message will not be restricted by the message length and can provide more information to the travelers. For example, instead of providing messages like "FREEWAY BLOCKED | AT [location] | USE OTHER ROUTES", we could provide more detailed and human speech like audio messages, such as "Major accident ahead at [location]. 3 lanes are closed. Please use other routes", even along with detailed information of potential alternatives, such as "Please use Exit 50 and use Route 50".

Second, the audio message will require much less "active" participation and will enable a true "mobile" application - meant to be used while a driver is traveling. This distinguishes it from current 511 applications that explicitly state they are to be used only when the vehicle is not in motion. Also, the drivers no longer need to be distracted by focusing their attention to read roadside messages signs and simply hearing the message will generally be much easier for the drivers.

(2) Scalability

A key concern is that DMSs are fixed assets. Each sign if expensive and can only provide information to travelers in a very small geographic region. Thus, they are located in limited locations and can only inform travelers at the location where they are installed. The VDMS is non-physical and can be "built" by defining information links or new lat/long areas from the server theoretically anywhere with only marginal cost, once the infrastructure and user-end devices become mature. For example, when an incident happens, instead of relying on several fixed DMSs or using limited number of portable DMSs, we could simply related the information to all the relevant links, and this is simple, quick and of very low cost.

\section{(3) Personalization}


Current DMSs are one for all, same font type, size, abbreviations and same display interval for two phase messages. They are suitable for most people, but not the best for all of them and not even suitable for small groups of people with special needs. When some drivers are unable to read English, current DMSs are completely not useful. The VDMS is able to present traffic messages in English, Spanish, and other languages. Also, features such as the audio volume, the audio pace, and times of repeating the same message should also be adjustable to fit the drivers' preference best. It is reasonable to believe that drivers will make better choices when they feel comfortable with the information sources and thus can receive and understand messages more easily.

\section{(4) Location-based}

The current public traffic information applications, such as the 511 website and smartphone app, usually provide users with information throughout a region or even a state. No matter where the incident occurs, the users will be given a message when using the app. Even if some new filtering functions are added such that users could input several of their usual routes (such as select "I-66"), the range is still too large and the messages are usually not relevant to a specific trip.

Overwhelming irrelevant information may reduce the users' interest and cause them to stop using the application. To avoid this problem, VDMS "places" traffic information on selected links or areas directly and travelers could receive the message only when they are on the link or in the area. Furthermore, different messages could be "placed" at one location but only most appropriate ones are given to a certain driver based on his characteristics, such as destination, value-of-time, and preferred routes. If a driver is not going to hit the downstream incident since his destination is close and he will use the next off-ramp, the incident information is not provided to avoid unnecessary distractions. 


\subsection{VDMS Smartphone Prototype}

The evaluation of this new concept of VDMS may require a lot of efforts from various perspectives. As a first step of the efforts, we hope to get an overall idea how this new concept will work and what the travelers' views and acceptance are. We decided to develop a prototype system, and ask users their opinions with a survey. Compared with DSRC, whose application in transportation systems are still in the initial stage of development, smartphone technology and cellular networks, especially 4G/LTE networks, have been well developed and applied for both collection and dissemination of traveler information for a long time. Smartphone technology has been used by many developers and researchers in recent transportation application development (e.g., Jin et al., 2012; Liao, 2013). Therefore, we decided to prototype and evaluate a smartphone VDMS application. In the prototype application, a traveler's location is monitored using the phone's GPS. When the traveler is in range of a pre-defined information area (i.e. in a range of latitude/longitudes), the information is presented to the traveler via an audible message.

\section{System Architecture}

The high-level conceptual diagram of the proposed Virtual DMS System is illustrated in Figure 3-1. It is designed to allow two-way communication between the smartphone and server. The five main components of the architecture are the client (cell phone), the cellular communication network, the Internet, the server and the DMS web service as in Figure 3-2. The location function of the smartphone will be activated once the application is on and will get the locations of the vehicle at certain intervals. These location data, along with time information, are then sent back to the server via cellular network (the second major component). The cellular network can also be used to provide user position information through cell signal triangulation or Cell-ID of the base station if the primary means of obtaining this data (i.e., the GPS satellites) is unavailable. The Internet, the third component, is the transit network for application data between the cellular 
network and the server. The next element of the system architecture is the server, which checks the real-time location information of each vehicle and sends back the relevant DMS message back to the user. The last element is the traffic information sources which is responsible for real-time traffic messages.
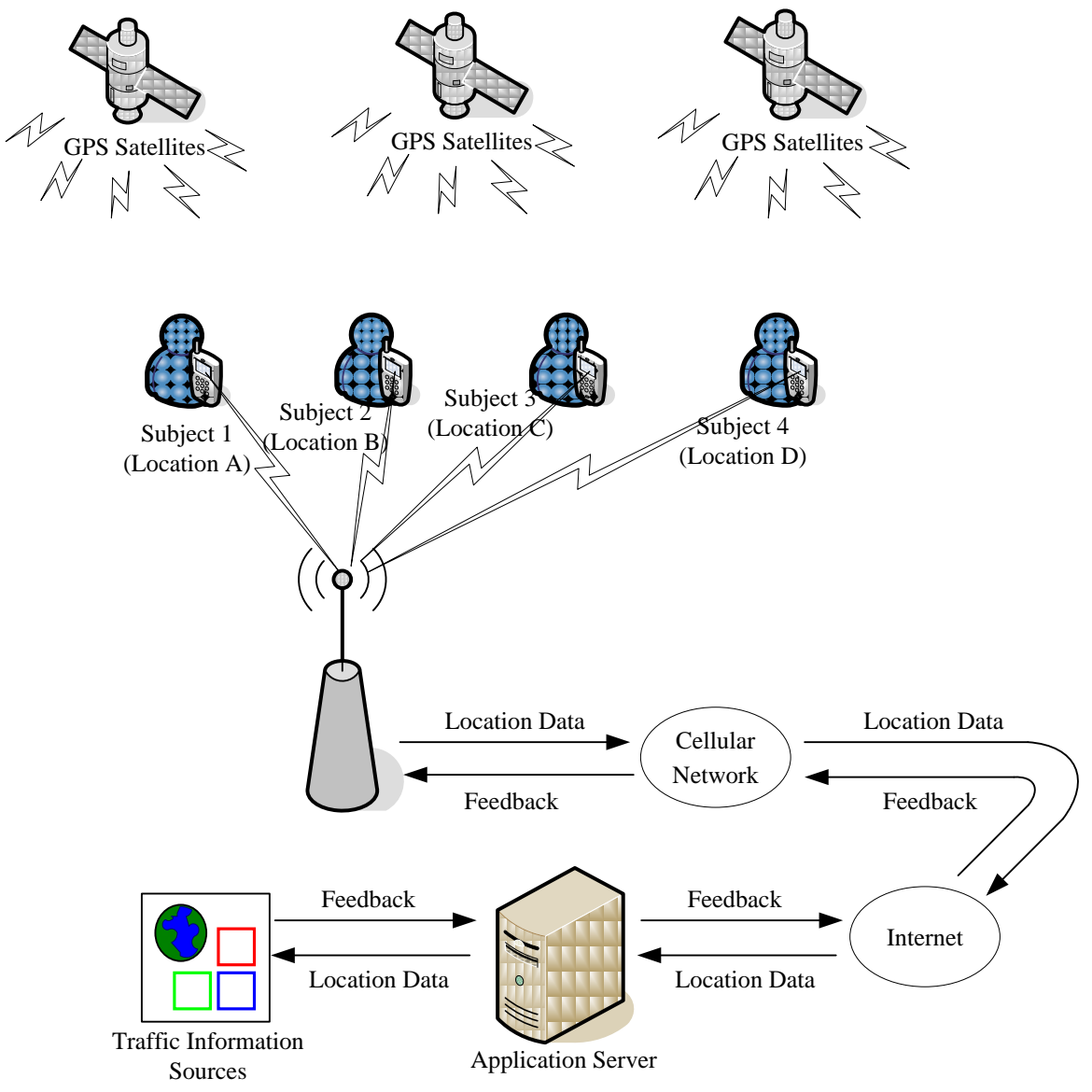

Figure 3-1 VDMS System Architecture 

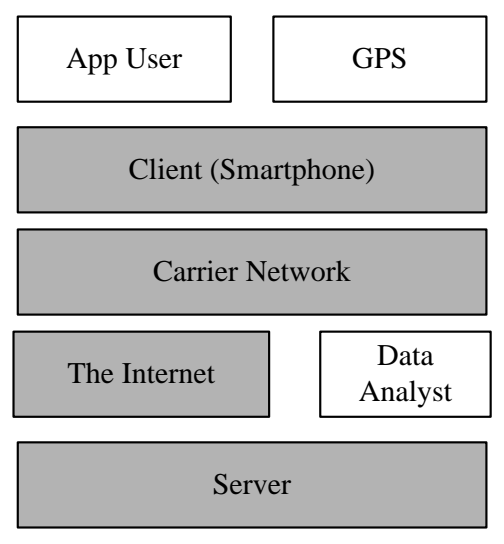

Traffic Information Sources

Figure 3-2 Main System Components of VDMS

\section{Implementation}

The VDMS application was developed on the Google Android platform for smartphones (http://developer.android.com/index.html). The app was developed with the JDK 1.6 and Android SDK4.1.2. The functionality of the smartphone application is to locate the vehicle on a regular basis and send this information to the server. After it receives feedback information from the server, the application will convert the text message to audio for the user. The location function is realized by using the standard "LocationListener" service provided by Android. The "LocationListener" is responsible for the requesting and obtaining of GPS information that can be used to derive coordinates, speed, heading and other position and travel data at present intervals. Android offers a Text-To-Speech (TTS) API that can be personalized through the language, pitch, and speech rate. This technology offers the opportunity to further improve safety and improve upon the current industry standard method of sending text messages for traffic alerts. In an application created using the Android Software Development Kit (SDK) and TTS API, messages sent to the smartphone are "spoken" to users. 
The server is responsible for receiving, analyzing, and providing feedback for travel data transmitted by the end-user device. An open source relational database management system (RDBMS), PostgreSQL, is used to store and process real-time data. These data include each GPS location, date and time stamp, travel direction, and speed, etc. and closest point on the link generated from the map matching process. PostGIS is a spatial database extender for the PostgreSQL object-relational database. It adds support for geographic objects allowing location queries to be run in SQL. We made use of PostGIS for spatial analysis, especially the map matching process. A server program was developed to match each point it receives to a server digital map (Northern Virginia Interstates network in this study) to find the exact link where the car is. Since the network we use in this study is relatively sparse and contains only freeways and arterials, a simpler mechanism of snapping to the closest link is used. Meanwhile, real-time traffic information is requested by the server from the Virginia Traffic and Video Data Sharing Site (VDOT TVD, vdotdatasharing.org).

The communication between the server and client is though the $3 \mathrm{G} / 4 \mathrm{G} / \mathrm{LTE}$ cellular network and the Internet. This "ServerSocket" class in the Android SDK is used and it represents a server-side socket that waits for incoming client connections. A "ServerSocket" handles the requests and sends back an appropriate reply.

\subsection{User Acceptance and Experience Survey}

A focused group user experience survey was conducted to evaluate the developed VDMS traveler information app to obtain user's potential attitude toward the new concept of transportation telematics. Since the experiment network for the prototype VDMS system uses Northern Virginia road network, 21 commuters in Northern Virginia (NOVA) were recruited through email blast and advertisement. The test network is the NOVA of freeway system, including I-66, I-495 and I- 
395. Participants are selected based on a few basic criteria (e.g., possessing an Android smartphone and commute on the NOVA test network daily). After that, each participant were provided with step-by-step instructions (http://people.virginia.edu/ jm7md/vdmssupport.html) to download the app from Google Play store (https://play.google.com/store/apps/details?id=edu.virginia.cts.vdms\&hl=en) and install the app on the personal Android phone. Each participant also received a free car-charger as an incentive to use the app for a relatively long time. Participants were required to use the app at least two weeks and then a survey link was sent to each of them to collect data on their acceptance level and experience with the app.

Among 21 participants, 13 of them (61.9\%) are male and 8 (38.1\%) are female. About $14.3 \%$ of them have some college level education, $38.1 \%$ are college graduate and $47.6 \%$ are postgraduate. In terms of age, $33.3 \%$ of them are between 18 and 29, $47.6 \%$ between 30 and 39 , $9.5 \%$ between 40 and 49 , and $9.5 \%$ between 50 and 64 . The demographic distribution shown above is generally balanced and meets the requirement considering the purpose of this study. The survey questions could be found in Appendix A.

The first question of the survey involves 9 sub-questions/items from two perspectives to assess the acceptance of the new application of transportation telematics: usefulness and satisfaction, according to Van Der Laan (1997). Questions are designed based on these two aspects and include 9 attributes, such as overall rate on the usefulness and pleasantness, safety, information value, convenience and easiness of receiving and understanding information, and annoying or not, etc. The aggregate survey results are shown in Table 3-1. Note that the number in each cell is the number of participants who checked the cell during the survey.

Table 3-1Aggregate Results of Nine-item Questions on User Acceptance Level

\begin{tabular}{|l|l|l|l|l|l|l|l|}
\hline & Answer Options & Strongly & Agree & Neutral & Disagree & Strongly & \\
\hline
\end{tabular}




\begin{tabular}{|l|l|l|l|l|l|l|l|}
\hline & & Agree & & & & Disagree & \\
\hline Score & & -2 & -1 & 0 & 1 & 2 & \\
\hline 1 & Useful & 5 & 11 & 4 & 0 & 1 & Useless \\
\hline 2 & Pleasant & 2 & 12 & 6 & 1 & 0 & Unpleasant \\
\hline 3 & Good & 0 & 14 & 6 & 1 & 0 & Bad \\
\hline 4 & Nice & 2 & 13 & 5 & 1 & 0 & Annoying \\
\hline 5 & Effective & 2 & 13 & 5 & 0 & 1 & Superfluous \\
\hline 6 & Likeable & 4 & 12 & 4 & 1 & 0 & Irritating \\
\hline 7 & Assisting & 5 & 12 & 3 & 0 & 1 & Worthless \\
\hline 8 & Desirable & 3 & 14 & 3 & 0 & 1 & Undesirable \\
\hline 9 & Raising alertness & 9 & 10 & 2 & 0 & 0 & Sleep-inducing \\
\hline
\end{tabular}

Recommended by Van Der Laan (1997), the following steps are adopted for the analysis.

1) For each of the nine questions, individual items are coded from -2 to +2 from left to right.

2) Perform reliability analyses. Use Question 1, 3, 5, 7, and 9 for the usefulness scale, and Question 2, 4, 6, and 8 for the satisfying scale.

3) If reliability (Cronbach's $\alpha$ ) is sufficiently high (above 0.65 ), compute per subject the end score for the two scales by averaging the scores on items $1,3,5,7$, and 9 for the usefulness score, and averaging scores on items 2, 4, 6, and 8 for the satisfying score.

4) The usefulness scale can now be averaged over subjects to obtain an overall system practical evaluation. The same is done with the satisfying scores.

Following the above steps, the Cronbach's $\alpha$ is calculated and the result of 0.93 indicates great reliability of answers in the survey. Then, the overall evaluation scores are calculated and the results are -0.90 and -0.81 for usefulness and satisfaction respectively. On a scale of -2 to 2 , the scores implies that on average, the participants "agree" with the usefulness of the new system and are satisfied with the experience of using the app as an tool to receive en-route DMS-like 
traveler information. Thus, we can conclude that the participants can accept this new type of enroute traveler information in terms of usefulness and satisfaction.

The next two questions are specific on the functionality of the developed app, regarding whether the provided information is relevant and timely respectively. The results, as shown in Table 3-2, indicate general agreement of users with the functionality of the app and confirm the feasibility of using the developed app for this study. Note that some participants gave low rating for the two questions and it could because that the app are designed to broadcast information about one mile ahead of the source DMSs. And sometimes the information of DMS could be problematic itself.

Table 3-2 Survey Results of Question 5 and 6

\begin{tabular}{|c|c|c|c|c|c|}
\hline & 1 & 2 & 3 & 4 & 5 \\
\hline \multirow{3}{*}{$\begin{array}{l}\text { Q5. Was the } \\
\text { information } \\
\text { provided } \\
\text { relevant? }\end{array}$} & Completely & & & & Completely \\
\hline & Disagree & & & & Agree \\
\hline & $2(9.5 \%)$ & $4(19.0 \%)$ & $5(23.8 \%)$ & $5(23.8 \%)$ & $5(23.8 \%)$ \\
\hline \multirow{2}{*}{$\begin{array}{l}\text { Q6. Was the } \\
\text { information } \\
\text { timely? }\end{array}$} & Really Bad & & & & Really Good \\
\hline & $1(4.8 \%)$ & $5(23.8 \%)$ & $5(23.8 \%)$ & $9(42.9 \%)$ & $1(4.8 \%)$ \\
\hline
\end{tabular}

Question 7 asked participants "Which message type would you prefer?". The result in Table 3-2 implies that the number of participants who prefer VDMS audio information is more than three times of DMS. Also, very few participants prefer text message on the phone, probably because participants also understand the inconvenience and safety concerns of reading text messages during driving. 


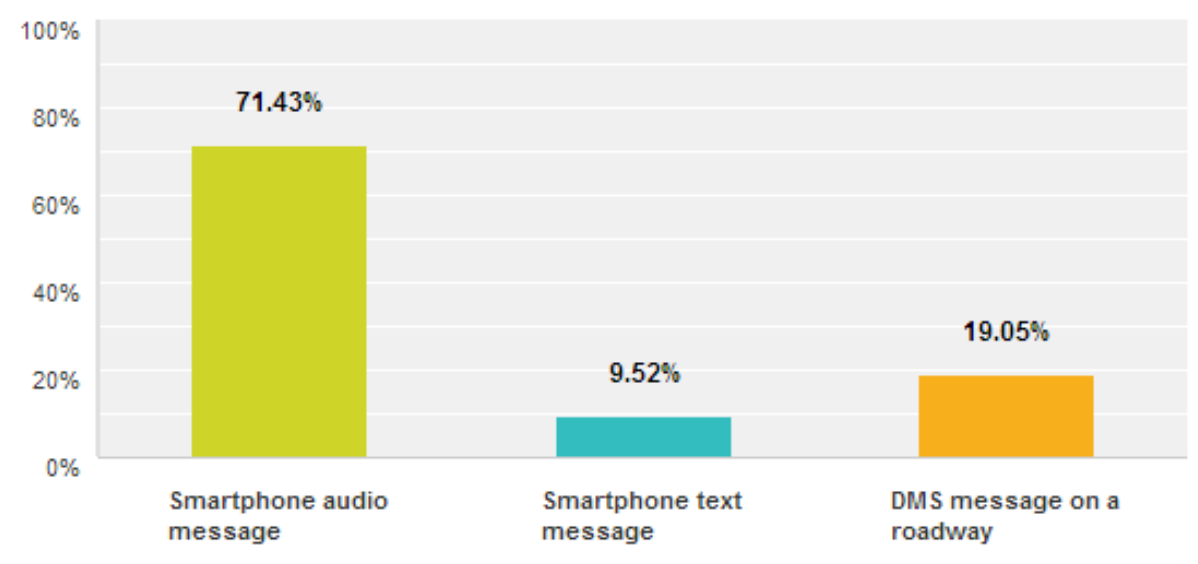

Figure 3-3 Answers for Question 7

The question Q8 asked participants "Do you feel it is safer to hear the audio messages on your phone or to read the messages posted on the DMS signs?". The result in Figure 3-4 clearly demonstrates that most of participants believe that VDMS audio message is safer way to receive en-route traffic information.

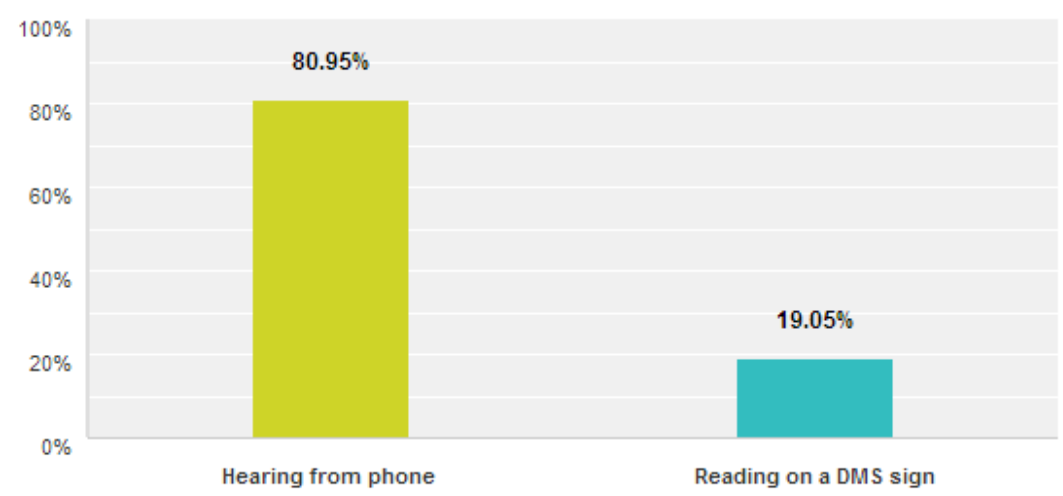

Figure 3-4 Answers for Question 8

The question Q9 asked about how comfortable participants felt in receiving the two types of information. The result in Figure 3-5 shows that twice of the participants felt more comfortable in hearing message from the phone than reading from roadside DMS signs. 


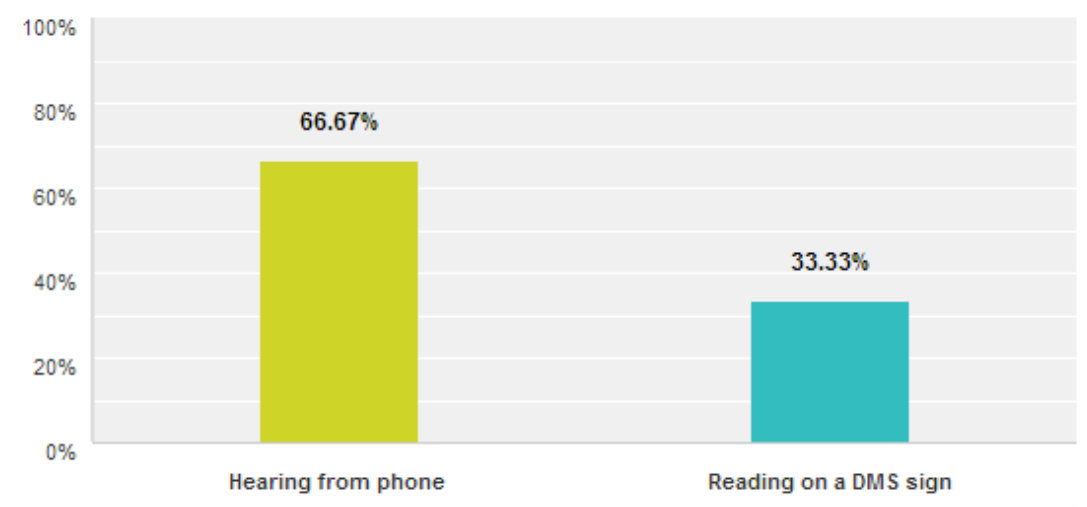

Figure 3-5 Answers for Question 9

The question Q10 asked participants whether they are more likely to follow suggestions from the audio message sent to your phone or from a roadside DMS posted message. It is expected that most of them don't distinguish them from each other as show in Figure 3-6. However, more participants chose VDMS instead of DMS probably because that they consider VDMS as a more personal source of information, to which they would better trust during their travel.

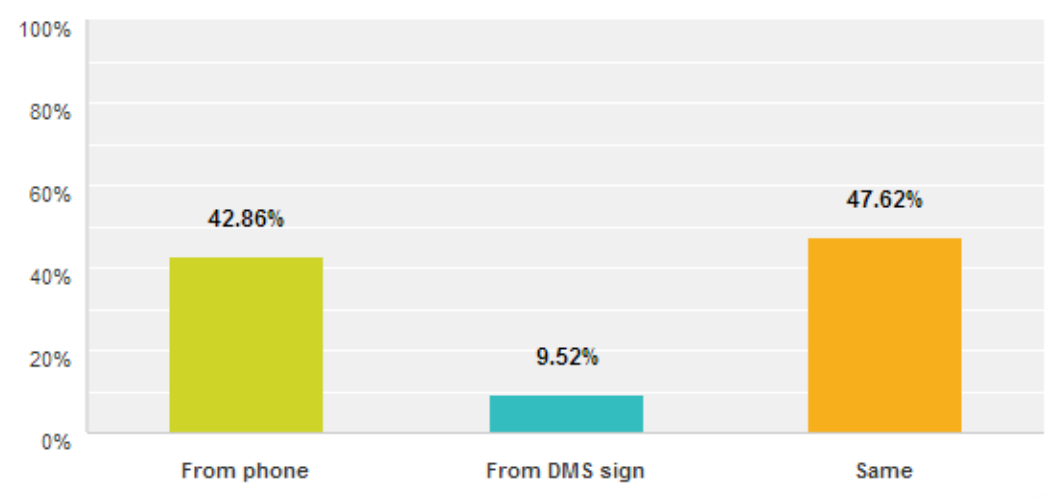

Figure 3-6 Answers for Question 10

The subsequent questions asked about opinions on the future functions and deployments. $85.7 \%$ of the participants prefer the audio message to be repeated twice. This is an expected result since repeated messages could increase users' comprehension of messages and this is also 
an advantage of VDMS over DMS, which has limited time (around 8 seconds) for users to read when they travel on freeways at $65 \mathrm{mph}$. Also, $81.0 \%$ participants prefer receiving messages at more locations to limited locations where DMS signs are currently located and this coincides with the scalability benefits of VDMS.

Since VDMS can potentially provide more information of different types, the next question asked about the preferred information contents users would like to hear from VDMS and the results are shown in Table 3-3. The ranking is from 1 to 7 (High to low) and the rating average is calculated by averaging all the ranking for the specific information type. As we can see, when more information can be potentially delivered, users prefer more comprehensive information to make a better choice decision. This coincides with the results of many studies (e.g., Peeta, 1995).

Table 3-3 Preferred Information Contents

\begin{tabular}{|c|c|c|c|c|c|c|c|c|}
\hline Answer Options & 1 & 2 & 3 & 4 & 5 & 6 & 7 & $\begin{array}{l}\text { Ranking } \\
\text { Average }\end{array}$ \\
\hline Occurrence and location of an incident & 1 & 0 & 3 & 5 & 8 & 3 & 1 & 4.52 \\
\hline $\begin{array}{l}\text { Occurrence and location of an incident }+ \text { Expected } \\
\text { delay (travel time) }+ \text { Detour strategy }\end{array}$ & 16 & 3 & 0 & 0 & 1 & 0 & 0 & 1.35 \\
\hline Expected delay (travel time) & 1 & 3 & 1 & 2 & 2 & 7 & 5 & 5 \\
\hline Detour strategy & 0 & 0 & 1 & 3 & 1 & 6 & 10 & 6 \\
\hline $\begin{array}{l}\text { Occurrence and location of an incident }+ \text { Expected } \\
\text { delay (travel time) }\end{array}$ & 1 & 6 & 9 & 3 & 2 & 0 & 0 & 2.95 \\
\hline $\begin{array}{l}\text { Occurrence and location of an incident }+ \text { a detour } \\
\text { strategy }\end{array}$ & 2 & 8 & 4 & 1 & 1 & 4 & 1 & 3.33 \\
\hline Expected delay (travel time) + a detour strategy & 0 & 1 & 3 & 7 & 6 & 1 & 3 & 4.57 \\
\hline
\end{tabular}

The last question asked about the possible reasons for which users may stop using such a system. The results are shown in Table 3-4. We can see that "Too much irrelevant information", 
"Negative impact on battery life" and "Negative impact on other applications" are the top three issues of great concern. The two comments made by two participants who selected "Other" are both related to message delay ("Timeliness of the announcements" and "Make sure information is current to make a good driving decision").

Table 3-4 Results of Survey Question 6 (reasons of opt-out)

\begin{tabular}{|l|l|}
\hline Q16. Under what circumstances will you opt out? \\
\hline Answer Options & $\begin{array}{l}\text { Response Count } \\
\text { (Percent) }\end{array}$ \\
\hline Too much irrelevant information & $7(33.3 \%)$ \\
\hline Frequent information is distracting & $1(4.8 \%)$ \\
\hline Low sound quality (noisy sound) & $0(0.0 \%)$ \\
\hline Negative impact on the battery life & $6(28.6 \%)$ \\
\hline $\begin{array}{l}\text { Negative impact on other applications running on the } \\
\text { phone }\end{array}$ & $5(23.8 \%)$ \\
\hline Other & $2(9.5 \%)$ \\
\hline
\end{tabular}

\subsection{Summary}

This chapter proposes the concept of Virtual Dynamic Message Sign (VDMS) as the next generation tool for DOTs to deliver en-route traveler information, and evaluate the concept with a smartphone application prototype system with a user survey. Several insights are offered in this process.

VDMS solves multiple flaws of DMS and other current en-route traveler information systems. Auditory messages could help deliver more detailed information and reduce driver distraction; and scalable location-based information could deliver more relevant information in a wider area with only marginal cost. 
A user experience survey reveals a relatively positive attitude among subjects toward VDMS in terms of both usefulness and satisfaction. The survey also indicates that drivers feel VDMS is a safer way to receive information and drivers feel more comfortable with VDMS compared with DMS.

Many research needs are also identified in this study, such as more detailed market research based on user experience. Also, issues like how to deliver auditory messages and whether they are effective compared with DMS in terms of message comprehension and distraction needs to be investigated with a more complex human factor study. 


\section{CHAPTER 4 HUMAN FACTORS: EFFECTIVENESS OF IN-VEHICLE AUDITORY MESSAGES}

On the basis of the preliminary results from Chapter 3, VDMS is a reasonable way of traveler information delivery in terms of user acceptance and stated preferences. In order to provide a solid foundation for state DOTs and TMCs for future deployment, a well-designed human factor study is needed to investigate key aspects of how drivers perform when they receive messages, such as message comprehension, driver distraction and subjective difficulty level they feel.

\subsection{Introduction}

Dynamic Message Signs (DMS) are the most widely used method to deliver public en-route traveler information by public agencies. As discussed in earlier chapters, DMSs are subject to many constraints, limiting the effectiveness. The proposed concept of VDMS is advantageous since it provides scalability for disseminating real-time traffic information at a large number of potential locations, without the infrastructure costs of installing regular DMSs. More importantly, it can potentially provide more detailed information, including basic messages and more advanced routing advisories under emergencies (e.g., alternatives). This paper will investigate whether VDMS can deliver information at least as well as current DMSs in order to determine if it is feasible for DOTs to deliver real-time traffic information using VDMS equipped in-vehicle devices in the future, especially when connected vehicles systems are in place. 
Although many studies have investigated human factor issues related to message design and display design of in-vehicle route guidance systems, this paper differs from them in several aspects. This system is proposed for use by DOTs to disseminate public traffic information, similar to current DMS messages instead of visual/auditory turn-by-turn information. The comprehension and distraction of navigation information is greatly different from that of a common DMS-type message. Also, since we want to provide public agencies reference for future development. We want to use the widely accepted DMS as the baseline and compare it with VDMS under the same experimental set-up.

\subsection{Objective and Scope}

A human factors study using a driving simulator was performed in order to assess whether there were significant differences between traffic messages presented on a simulated DMS and VDMS. The specific objectives of this paper are to:

- Determine whether VDMS can reduce driver distraction relative to DMSs

- Determine the comprehension of VDMS messages relative to DMS messages

- Determine subjective difficulty level drivers feel when receiving messages from VDMS compared with DMS

\subsection{Methodology}

A driving simulator-based human factor study was conducted to assess driver performance and preference between the proposed VDMS system and a simulated DMS. While real-world tests offer physical, perceptual, and behavioral fidelity, they are limited in terms of experimental controllability, reproducibility, and standardization. Meanwhile, driving simulators provide 
flexibility in experimental design and control and help to maintain consistency and reduce confounding factors. Driving simulators can also provide richer driver performance data than can typically can be obtained in field tests.

\subsubsection{Driving Simulator Environment}

The experiment was conducted using the Driver Guidance System (DGS, https://www.generalsimulation.com) at the University of Virginia. DGS is composed of simulation terminals and a data center with analysis tools to assess driving ability. The DGS collects driving performance data using a variety of driving scenarios and transmits the data to the data center for analysis, as shown in Figure 4-1 (a). As in Figure 4-1 (b), the simulator terminal has a field of view subtended by approximately 200 degrees and an angular distortion error target of less than 1.5 degrees. The data is captured sixty times per second $(60 \mathrm{~Hz})$ and all control manipulations, position and orientation of vehicles are captured. Figure 4-1 (c) shows the real experimental set-up and equipments. The messages in yellow and pixilated font in a dark grey box are projected to the screen using another projector. The messages looks more clearly in reality than in the picture and all participants reported that they can easily see the messages without extra efforts. 


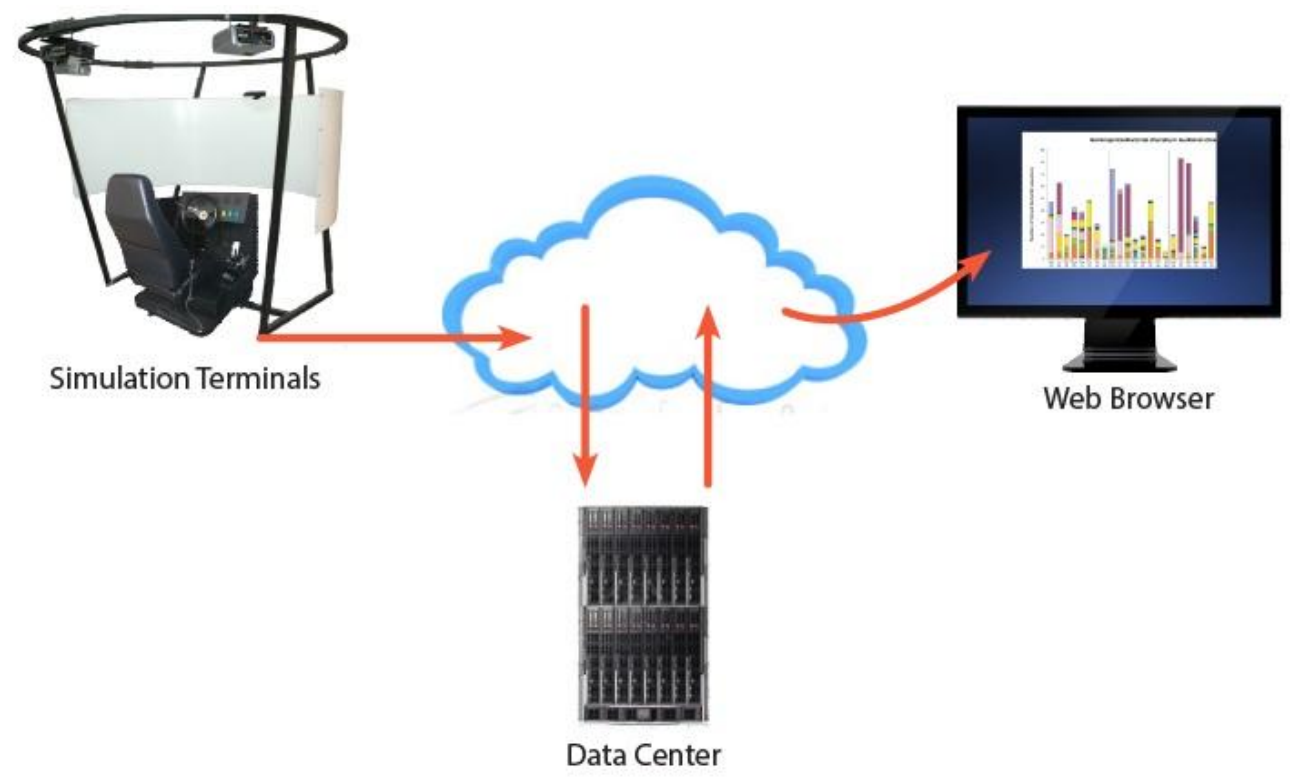

(a) DGS components and data flow

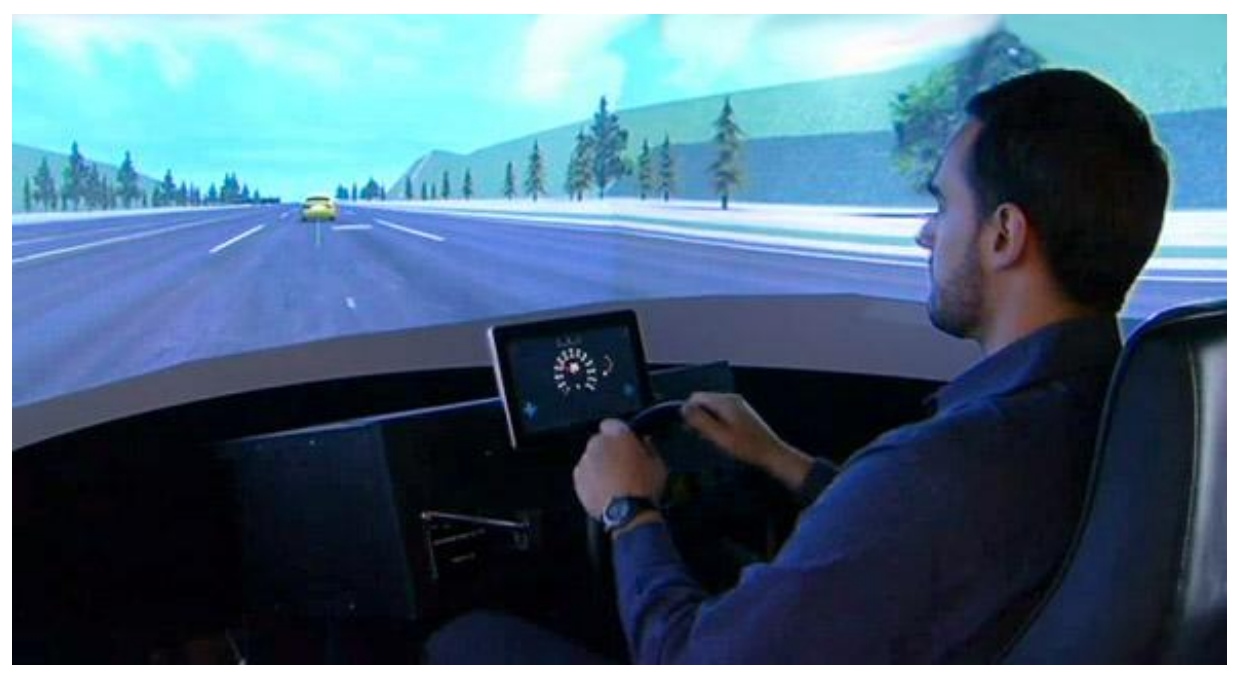

(b) Driving simulator (car-following scenario) 


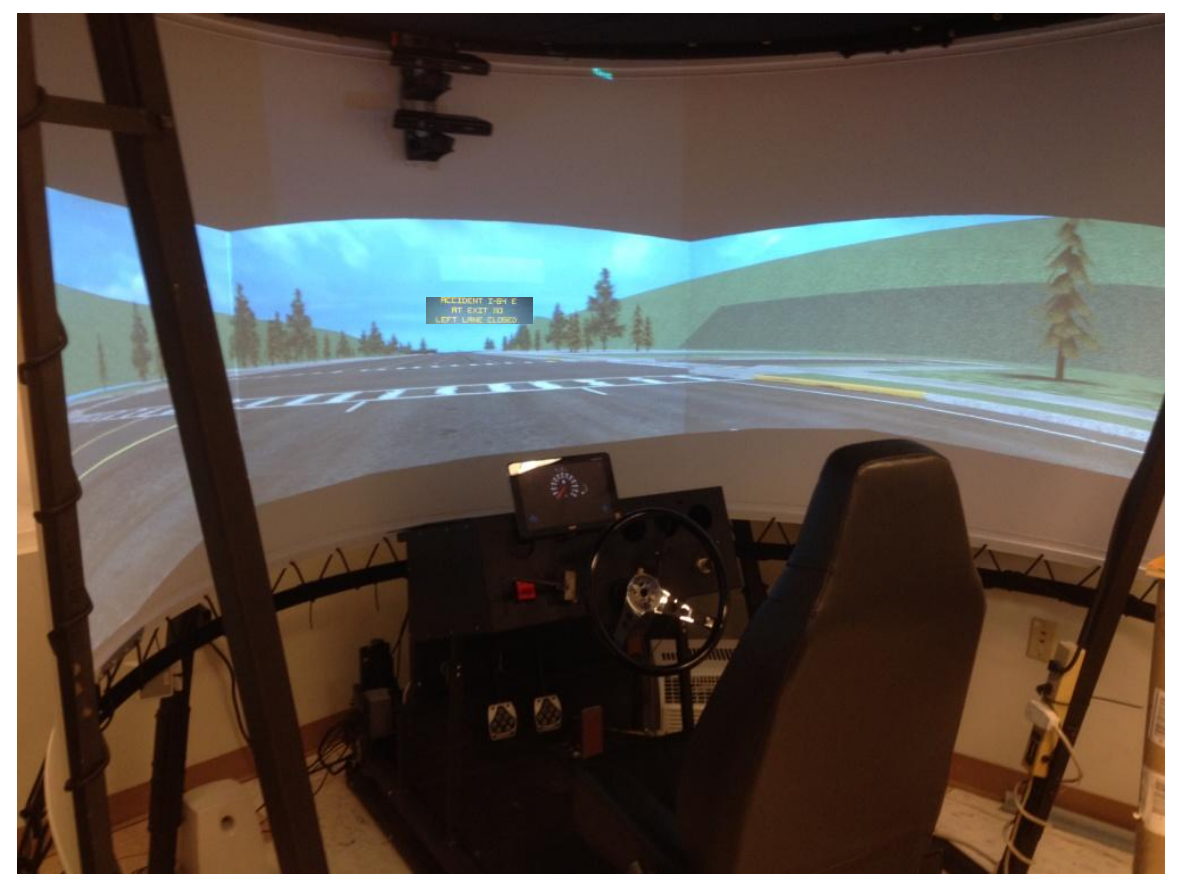

(c) Picture of Experimental Set-up and Equipments (note simulated DMS in middle of screen)

Figure 4-1 Driver Guidance System (DGS, https://www.generalsimulation.com)

\subsubsection{Test Factors and Experimental Design}

Message comprehension and driver distraction are two most important measures of effectiveness when evaluating traveler information systems. Also, it is desirable that drivers subjectively feel comfortable with the traveler information that is being provided. In order to investigate these measures, an experimental design was created to investigate four factors that could influence driver comprehension and performance:

- Information mode ("Mode", 2 levels)

○ DMS (text, level=1)

○ VDMS (audio, level =2)

- Information amount (“Amount”, 2 levels)

$\bigcirc$ Low information load (3 units of information, level=1) 
- High information load (6 units of information, two-phases for DMS display, level =2)

- Driving condition (“Driving”, 2 levels)

○ Simple driving scenario (level=1)

- Complex driving scenario (level $=2$ )

- Participant age (“Age”, 2 levels)

○ Young (18-60 years old, level=1)

○ Old ( $>60$ years old, level $=2$ )

The DMS messages, as shown in Figure 1(c), were displayed in the center of on the driving simulator screen for all scenarios using a separate projector connected to a laptop computer. Yellow characters in pixilated font are used to display experiment DMS messages to mimic real world signs. Auditory messages were also delivered using the laptop computer and a separate speaker, placed next to the driving simulator. Also, the simulator will generate engine noise based on the speed of the vehicle.

The definition of information units used in this study is consistent with the tenets advanced by Dudek and Huchingson (2006) for DMS messages in the Manual on Real-Time Motorist Information Displays. The term generally means information that can be used to answer a simple question, such as "What is the traffic problem?", "Where is the traffic problem located?", and "What was told about the lanes?". Also, messages for DMS and VDMS in this example are same to avoid confounding factors.

The system has two pre-programmed scenarios that were used to represent simple and complex driving environments. The first is a car following scenario that is typical of a simple daily driving task. The participants need to follow a lead vehicle at a constant speed, and brake when the lead vehicle's brake light is activated. The timing and number of braking activations of 
the lead car is random for each scenario and is also dependent on participants' driving behavior (If the driver never starts driving, the lead vehicle will wait for the following car and braking scenarios will never occur). The distance between two vehicles is always the same for all brake activations during different scenarios. The simulation will adjust itself if drivers brake slowly or fast and even fail to brake at any moment during the simulation. The brake light of the lead vehicle is only activated when a message has started to be broadcast or displayed. With these settings, it is difficult for participants to anticipate when the brake lights will be activated. Also, there are short and long brake activations and they are randomly presented. It is unlikely that participants may opt to take no action (i.e. not respond) since they cannot predict that the lead car braking action would be only a momentary occurrence or long brake. For long brake activations, participants have to brake to a stop to avoid crashing into the lead car.

The second scenario involved car following while simultaneously avoiding potholes that are randomly shown on the road. Potholes are added in addition to the brake light activations in the first driving scenario and are used to represent more complex driving conditions. Potholes are 3-dimensional objects that are randomly shown on the ground one after another, and riding over them will cause obvious shaking and sound in the simulator. Drivers are told to avoid all potholes. Note that the location of potholes is different in each scenario, depending on actual participants' driver behavior and scenario set-up. For example, if a driver cannot drive in the center of a specified lane, the potholes will never show up.

A four-way mixed design (or partially repeated measure design, Edelman et al. [2009]) was used in this study. The repeated measure design was used for two reasons. First, there may be a great deal of variation between participants, such as self-reported message difficulty level, which may highly depend on the subject's characteristics. Then, error variance estimates from standard ANOVAs are large. Repeated measures of each participant provide a way of accounting for this variance, thus reducing error variance. 
The use of mixed design is because that while Information Mode, Information Amount and Driving Condition are within subject independent variables, Participant Age is a between subject independent variable. There are two levels for each factor, so the total number of different scenarios will be $2 \times 2 \times 2=8$. Also, 2 replications were conducted for each scenario for each participant, which means that each participant went through was $8 \times 2=16$ scenarios. The simulated driving environments of the 16 scenarios are very similar to each other, with only minor differences in surrounding environment, existence of potholes, timing of brake light activations, and timing of message. This is done to avoid introduction of other confounding factors. In order to avoid practice and fatigue effects, the order of each scenario was randomly generated for each of the participants. This randomization also served to mitigate fatigue effects that could occur for scenarios tested at the end of the session. Details of the 16 scenarios and experimental messages are shown in Appendix B.

\subsubsection{Participants}

All participants were required to have a valid driver's license and drive at least 1,000 miles/year on freeways. Since this study wants to examine the effect of Age as a factor, both young and older participants were recruited.

The statistical software G Power 3 (Faul,2007) was used to calculate sample size. The software permits a priori power analysis (i.e., calculate sample size for given effect size, alpha, power, and design). Based on the recommendation of Cohen (1999), several assumptions of required parameters were made: median effect size $(0.25)$, power value $(0.8)$, correlation among repeated measures (0.5), and nonsphericity correction (1, for within-between factor interaction). The required sample sizes for between factors, within factors, and within-between interactions are calculated separately and the values of above assumptions with median effect size are 22, 10 and 
12. For example, when we assume a smaller effect size of 0.20 , the required sample size becomes 34. To account for the possibility of small effect size and also consider time and resources available, 46 participants were recruited. Four of them were not able to finish the experiment due to simulation sickness. Therefore, data analysis was conducted with the remaining 42 participants (27 young and 15 old, 20 male and 22 female), with age ranges from 19 to 73 . The mean age for the young group is $25.6(\mathrm{SD}=5.58)$ and the mean age of older group is $65.3(\mathrm{SD}=6.58)$. All of the participants met the basic eligibility requirements and none of the participants reported having any vision and hearing deficiencies.

\subsubsection{Experimental Procedure}

Each participant was tested using the same procedures. First, written instructions were read to each participant. They were instructed on the goals of the study, told about the driving simulator, and told about compensation procedures. Next, each participant was given ten to fifteen minutes to get familiar with the driving environment before the test started. This was done to reduce testing bias related to lack of driver familiarity with the simulator. The practice driving scenarios are quite similar to the formal test environment. This is done to reduce the impact of unfamiliarity with the simulator that could be manifested during the initial runs for each subject.

The testing phase then began. Each participant was tested using the 16 scenarios in randomized order. Each scenario lasted about 2-3 minutes. The entire set of 16 scenarios was completed in approximately 40-50 minutes. In each scenario, participants drove one of the two driving conditions (simple or complex) and received one type of traffic message (DMS/VDMS

and short/long message). These messages were very similar to current DMS traffic messages used by DOTs, such as "Crash Ahead, Interstate 64 Eastbound Exit 112, Right lane closed.". 
Sometimes the message might also contain travel time information, such as "To US 29, 20 minutes via I-64".

Note that since the experiment was conducted in Charlottesville, VA, every message used in this experiment used local routes in the messages that were tested. Before the experiment was conducted, participants were given a map and asked to imagine that they are driving at a location before a diversion point. After they saw or heard a message, the experimenter would wait for another 20 seconds before asking participants open-ended questions for each information units, such as "What is the traffic problem?" and "What is the travel time to US 20 via I-64?". Each participant also needed to self-report the difficulty level of processing the messages in each scenario under the corresponding driving condition on a five point Likert scale ("1"-"Not difficult”, “2”-“A little”, ”3”-“Medium”, “4”-“Relatively”, and '5'-‘Very').

\subsubsection{Evaluation Metrics and Data Analysis}

Several evaluation metrics were used to evaluate driver performance in terms of message comprehension, driver distraction, and preference. Message comprehension was measured based on participants' answers to open-ended questions asked by the experimenter. The proportion of questions correctly answered corresponding to message information units was the performance measure. For example, correctly answering three questions out of five would be assigned a comprehension value of $3 / 5=0.6$.

Time-to-brake was used as a surrogate measure for level of driver distraction. This measure was computed by the simulator as the time that elapsed between when the lead vehicle's brake lights activated and when the driver began to step on the brake. This measure was used to assess whether there may be any adverse safety impacts for different factor combinations. 
Finally, self-reported difficulty in processing each message under each specific driving condition was also collected. This difficulty level was reported using a five-point Likert scale (scale "1" as "Not difficult", scale "3" as "Medium difficulty level", and scale "5" as "Very difficult"). This is a comprehensive measure that could include factors like distraction, preference, and ability of each participant to process visual/audio messages.

To sum up, the evaluation metrics used in this study were as follows:

- Message understanding based on the proportion of the open-ended questions that are correctly answered (interval)

- Driver distraction based on time-to-brake (interval)

- Subjective message difficulty level rating based on the five-point Likert scale (ordinal)

To examine the effects of the experimental conditions, mixed ANOVAs were applied. As described above, a value between 0 and 1 was assigned based on the percentage of messages that are comprehended, and, in this way, the general framework of mixed ANOVA was used to analyze this metric as well. Gene Glass et al. (1972) did a well-known Monte Carlo study of ANOVA and it showed that the F-test was incredibly robust to violations of the interval data assumption (as well as moderate skewing) and could be used to do statistical tests at the scale level of the data that was collected using a 5 to 7 point Likert response format with no resulting bias. Many other studies also confirmed the conclusion and applied this result into their application (Carifio \& Perla, 2007; Unal et al., 2013). Therefore, ANOVA is also applied in this study for analysis of Likert scale data.

A mixed four- way ANOVA using the generalized linear model (GLM) function in SPSS, was carried out to analyze the experimental data. Repeated measures ANOVA carries the standard set of assumptions associated with an ordinary analysis of variance, extended to the 
matrix case: multivariate normality, homogeneity of covariance matrices, and independence. Repeated measures ANOVA is robust to violations of the first two assumptions. Violations of independence produce a nonnormal distribution of the residuals, which results in invalid $\mathrm{F}$ ratios. This study selected participants randomly and the independence assumption should be fulfilled. (Edelman et al., 2009).

\subsection{Results}

\subsubsection{Message Comprehension}

GLM was run using message comprehension data (proportion of questions that are correctly answered) collected by the experimenter with open-ended questions. This measure reflects the percentage of information that participants correctly processed and retained. The main effects, two-factor interactions, and significant three-factor interaction results are shown in Table 4-1. Note that other three factor interaction and four factor interaction effects are not significant and thus not listed in the table for concision. Multivariate test statistics, such as Pillai's Trace and Wilks' Lambda, are usually used due to their robustness and the former is used in this paper (Cohen, 1996).

Table 4-1 Analysis Results for Message Comprehension

(a) Mixed ANOVA test results for message comprehension

\begin{tabular}{|l|l|l|l|l|}
\hline & \multicolumn{1}{|c|}{ F } & df & \multicolumn{1}{c|}{ Sig. } & $\begin{array}{c}\text { Partial Eta } \\
\text { Squared }\end{array}$ \\
\hline Within-subjects effects & 40.386 & 1 & .000 & .502 \\
\hline Mode & 102.689 & 1 & .000 & .720 \\
\hline Amount & 10.079 & 1 & .003 & .201 \\
\hline Driving & 2.222 & 1 & .144 & .053 \\
\hline Mode*Amount & 12.797 & 1 & .001 & .242 \\
\hline Mode*Driving & & & & \\
\hline
\end{tabular}




\begin{tabular}{|l|l|l|l|l|}
\hline Amount*Driving & 2.740 & 1 & .106 & .064 \\
\hline Mode*Amount*Driving & 22.123 & 1 & .000 & .356 \\
\hline Between-subjects effects & 8.102 & 1 & .007 & .168 \\
\hline Age & 1.135 & 1 & .293 & .028 \\
\hline Within-between interaction effects & 1.141 & 1 & .292 & .028 \\
\hline Mode*Age & .899 & 1 & .349 & .022 \\
\hline Amount*Age & & & \\
\hline Driving*Age &
\end{tabular}

(b) Post-hoc comparisons of factors Mode, Amount and Driving for message comprehension

\begin{tabular}{|c|c|c|c|c|c|c|c|c|}
\hline \multirow[b]{2}{*}{ Amount } & \multirow[b]{2}{*}{ Driving } & \multirow[b]{2}{*}{ (I) Mode } & \multirow{2}{*}{$\begin{array}{l}(\mathrm{J}) \\
\text { Mode }\end{array}$} & \multirow{2}{*}{$\begin{array}{c}\text { Mean } \\
\text { Difference } \\
\text { (I-J) }\end{array}$} & \multirow{2}{*}{$\begin{array}{l}\text { Std. } \\
\text { Error }\end{array}$} & \multirow[b]{2}{*}{ Sig. } & \multicolumn{2}{|c|}{$\begin{array}{c}\text { 95\% Confidence Interval for } \\
\text { Difference }\end{array}$} \\
\hline & & & & & & & Lower Bound & Upper Bound \\
\hline \multirow[t]{2}{*}{1} & 1 & 1 & 2 & .034 & .027 & .218 & -.021 & .089 \\
\hline & 2 & 1 & 2 & -.208 & .035 & .000 & -.280 & -.137 \\
\hline \multirow[t]{2}{*}{2} & 1 & 1 & 2 & -.173 & .036 & .000 & -.246 & -.100 \\
\hline & 2 & 1 & 2 & -.102 & .028 & .001 & -.158 & -.046 \\
\hline \multirow[b]{2}{*}{ Mode } & \multirow[b]{2}{*}{ Driving } & \multirow{2}{*}{$\begin{array}{l}\text { (I) } \\
\text { Amount }\end{array}$} & \multirow{2}{*}{$\begin{array}{l}\text { (J) } \\
\text { Amount }\end{array}$} & \multirow{2}{*}{$\begin{array}{c}\text { Mean } \\
\text { Difference } \\
\text { (I-J) }\end{array}$} & \multirow{2}{*}{$\begin{array}{l}\text { Std. } \\
\text { Error }\end{array}$} & \multirow[b]{2}{*}{ Sig. } & \multicolumn{2}{|c|}{$\begin{array}{c}\text { 95\% Confidence Interval for } \\
\text { Difference }\end{array}$} \\
\hline & & & & & & & Lower Bound & Upper Bound \\
\hline \multirow[t]{2}{*}{1} & 1 & 1 & 2 & .291 & .041 & .000 & .208 & .374 \\
\hline & 2 & 1 & 2 & .185 & .037 & .000 & .111 & .258 \\
\hline \multirow[t]{2}{*}{2} & 1 & 1 & 2 & .084 & .036 & .024 & .012 & .156 \\
\hline & 2 & 1 & 2 & .291 & .026 & .000 & .239 & .343 \\
\hline \multirow[b]{2}{*}{ Mode } & \multirow[b]{2}{*}{ Amount } & \multirow{2}{*}{$\begin{array}{l}\text { (I) } \\
\text { Driving }\end{array}$} & \multirow{2}{*}{$\begin{array}{l}(\mathbf{J}) \\
\text { Driving }\end{array}$} & \multirow{2}{*}{$\begin{array}{c}\text { Mean } \\
\text { Difference } \\
\text { (I-J) }\end{array}$} & \multirow{2}{*}{$\begin{array}{l}\text { Std. } \\
\text { Error }\end{array}$} & \multirow[b]{2}{*}{ Sig. } & \multicolumn{2}{|c|}{$\begin{array}{c}\text { 95\% Confidence Interval for } \\
\text { Difference }\end{array}$} \\
\hline & & & & & & & Lower Bound & Upper Bound \\
\hline \multirow[t]{2}{*}{1} & 1 & 1 & 2 & .153 & .041 & .001 & .071 & .236 \\
\hline & 2 & 1 & 2 & .047 & .029 & .110 & -.011 & .106 \\
\hline \multirow[t]{2}{*}{2} & 1 & 1 & 2 & -.089 & .030 & .005 & -.149 & -.029 \\
\hline & 2 & 1 & 2 & .119 & .024 & .000 & .071 & .166 \\
\hline
\end{tabular}

*Mode 1: DMS; Mode 2: VDMS; Amount 1: three Units of information; Amount 2: six units of information; Driving 1: Low complexity; Driving 2: High complexity. 
The results reveal significant main effects for Mode, Information Amount, and Driving Complexity. Within-subject interactions between Mode and Driving and Mode, Driving, and Amount were also significant. Other interaction effects between within-subject factors and within-between interaction effects are not statistically significant. Note that the Partial Eta Squared in Table 4-1 (a) (and other similar tables in this paper) is the statistic used to indicate the calculated effect size $(0.01=$ small, $0.06=$ medium, $0.13=$ large, according to Cohen [1996]). We can see that all the significant main or interaction effects have a large effect size and should be fully considered in the information system design.

Due to the significant interaction effects, we further conduct pairwise comparison as in Table 4-1(b). As shown in the first block of Table 4-1 (b), the effect of Mode is not significant only when driving complexity and message length are both low, and is significant for all other combinations. This means that while DMS and VDMS perform similarly under less demanding conditions, VDMS comprehension is significantly better than DMS under complex conditions or for longer messages regardless of driving complexity. On average, the comprehension rate of VDMS is $16 \%$ higher than DMS under these conditions.

The second block of Table 4-1 (b) reveals the effect of amount of information. For DMSs, longer messages reduce comprehension level, which is expected since it is more difficult for drivers to read and process longer messages within a specific time. However, this is true for VDMS only under complex driving conditions, and there is no significant difference between the comprehension of short and long messages under simple driving conditions. The means that under simple driving conditions, it is more viable to provide more information with VDMS since it will not significantly reduce message comprehension. 
The effect of driving complexity is shown in the third block of Table 4-1 (b). Generally, complex driving conditions cause lower comprehension levels of messages than simple driving conditions. The one exception is DMS with higher information content, possibly because the effect of more information is larger than the effect of more complex driving conditions, partially demonstrated by the value of Partial Eta Squared in Table 4-1 (a) (Amount=0.702, Driving=0.201). Although both of them are larger than 0.14 (and this are of large effect size), the effect size of amount of information is more than three times of driving complexity. The betweensubject variable, Age, is also significant at a 95\% level, and there are no significant interaction effects between between-subject and within-subject factors. This indicates that older drivers cannot process and retain as much information as young drivers in all conditions under our experimental settings. This indicates that both DMS and VDMS should be designed in a way that is still acceptable for older drivers. In particular, non-significant interaction between Mode and Age indicates that the effect of Mode is not dependent on Age. This means that all the main effects obtained above in this section apply to both young and old drivers. For example, VDMS is significantly better than DMS for both young and old drivers under relatively complicated conditions in terms of either information load or driving complexity.

\subsubsection{Driver Distraction}

Driver distraction was measured by the time-to-brake, which provides a surrogate measure for the safety effects of each factor. The main effects, two-factor interactions, and significant threefactor interaction results using Wilks' Lambda are shown in Table 4-2 (a). Note that other three factor interaction and four factor interaction effects are not significant and thus are not listed in the table for the sake of conciseness.

Table 4-2 Analysis Results for Distraction 
(a) Mixed ANOVA test results for distraction using time-to-brake data

\begin{tabular}{|c|c|c|c|c|}
\hline & $\mathbf{F}$ & df & Sig. & $\begin{array}{c}\text { Partial Eta } \\
\text { Squared }\end{array}$ \\
\hline \multicolumn{5}{|l|}{ Within-subjects effects } \\
\hline Mode & 204.537 & 1 & .000 & .836 \\
\hline Amount & .739 & 1 & .395 & .018 \\
\hline Driving & 17.710 & 1 & .000 & .307 \\
\hline Mode*Amount & .037 & 1 & .849 & .001 \\
\hline Mode*Driving & 8.941 & 1 & .005 & .183 \\
\hline Amount*Driving & .763 & 1 & .388 & .019 \\
\hline Mode*Amount*Driving & .021 & 1 & .886 & .001 \\
\hline \multicolumn{5}{|l|}{ Between-subjects effects } \\
\hline Age & .893 & 1 & .350 & .022 \\
\hline \multicolumn{5}{|c|}{ Within-between interaction effects } \\
\hline Mode*Age & 0.967 & 1 & .331 & .024 \\
\hline Amount*Age & .041 & 1 & .840 & .001 \\
\hline Driving*Age & 1.092 & 1 & .302 & .027 \\
\hline
\end{tabular}

(b) Post-hoc comparisons of factors Mode and Driving for distraction

\begin{tabular}{|c|c|c|c|c|c|c|c|}
\hline \multirow[b]{2}{*}{ Driving } & \multirow{2}{*}{$\begin{array}{l}\text { Mode } \\
\text { (I) }\end{array}$} & \multirow[b]{2}{*}{ Mode $(\mathbf{J})$} & \multirow{2}{*}{$\begin{array}{c}\text { Mean } \\
\text { Difference } \\
\text { (I-J) }\end{array}$} & \multirow[b]{2}{*}{ Std. Error } & \multirow[b]{2}{*}{ Sig. } & \multicolumn{2}{|c|}{$\begin{array}{c}\text { 95\% Confidence Interval for } \\
\text { Difference }^{\mathrm{a}}\end{array}$} \\
\hline & & & & & & Lower Bound & Upper Bound \\
\hline 1 & 1 & 2 & $.308^{*}$ & .036 & .000 & .236 & .380 \\
\hline 2 & 1 & 2 & $.475^{*}$ & .042 & .000 & .390 & .560 \\
\hline Mode & $\begin{array}{l}\text { Driving } \\
\text { (I) }\end{array}$ & $\begin{array}{l}\text { Driving } \\
(\mathbf{J})\end{array}$ & $\begin{array}{c}\text { Mean } \\
\text { Difference } \\
\text { (I-J) }\end{array}$ & Std. Error & Sig. & \multicolumn{2}{|c|}{$\begin{array}{c}\text { 95\% Confidence Interval for } \\
\text { Difference }^{\mathrm{a}}\end{array}$} \\
\hline 1 & 1 & 2 & $-.194^{*}$ & .050 & .000 & -.295 & -.094 \\
\hline 2 & 1 & 2 & -.027 & .022 & .218 & -.071 & .017 \\
\hline
\end{tabular}

*Mode 1: DMS; Mode 2: VDMS; Driving 1: Low complexity; Driving 2: High complexity.

The results reveal significant effects for two main within-subject factors: Mode and Driving, and interaction effects between Mode and Driving. The information amount factor is not 
significant. This may be a case of the driver prioritizing safe control of the vehicle over traveler information.

There are significant interaction effects between Mode and Driving, so caution should be used when interpreting the main effects of those factors. Post-hoc pairwise comparisons for the effects of Mode*Driving are shown in Table 4-2 (b). Under both simple and complex driving conditions, the effect of Mode is statistically significant. This confirms the significant main effect of Mode, and shows that the time-to-brake for VDMS is on average 0.39 seconds shorter than for DMS. This implies that VDMS is has less of an effect in terms of distraction level under both simple and complex driving conditions than DMS. However, the effect of driving complexity is only significant for the DMS mode and not for VDMS ( $\mathrm{p}=0.218)$. This indicates that the complex driving condition significantly increases the DMS message distraction level, while distraction level was not significantly increased by more complex driving conditions for VDMS messages. Since traffic messages are often provided during complex traffic conditions created by congestion or incidents, this result reveals another potential benefit of VDMS over DMS.

The main effect of the between-subject variable Age and interaction effects between within-subject effects and Age are not statistically significant, as shown in Table 4-2 (a). We can conclude that there is no significant difference between young and old drivers in their time to brake while processing traffic messages.

In addition to time to brake, the number of times when participants struck the lead vehicle was also recorded. In total, among the 672 discrete scenarios (16 for each of 42 participants), there were 11 crashes during DMS scenarios and 3 crashes for VDMS scenarios. Though the data is not large enough to test for statistical significance, the large difference in crash frequency may be an indicator of the potential benefit of VDMS in reducing distraction. 


\subsubsection{Perceived Difficulty}

A mixed ANOVA was run using the participants' Likert difficulty rating data for each scenario. This measure examines the perceived difficulty for the driver to process and retain messages under different driving conditions. This is a subjective measure, but drivers' perceptions may greatly affect the perceived effectiveness of a traveler information system. The main effects, twofactor interaction, and significant three-factor interaction results based on difficulty level data are shown in Table 3. Note that other three factor interaction and four factor interaction effects are not significant and thus not listed in the table for concision. Again Wilks' Lambda is used here to test for significance.

Table 4-3 Analysis results of Perceived Difficulty

(a) Mixed ANOVA test results for distraction using Likert difficulty rating

\begin{tabular}{|l|l|l|l|l|l|}
\hline & \multicolumn{1}{|c|}{ F } & df & \multicolumn{2}{c|}{ Sig. } & \multicolumn{1}{c|}{$\begin{array}{c}\text { Partial Eta } \\
\text { Squared }\end{array}$} \\
\hline Within-subjects effects & 41.642 & 1 & .000 & .510 \\
\hline Mode & 163.020 & 1 & .000 & .803 \\
\hline Amount & 12.164 & 1 & .001 & .233 \\
\hline Driving & 3.906 & 1 & .055 & .089 \\
\hline Mode*Amount & 12.727 & 1 & .001 & .241 \\
\hline Mode*Driving & 6.676 & 1 & .014 & .143 \\
\hline Amount*Driving & 28.408 & 1 & .000 & .415 \\
\hline Mode*Amount*Driving & 17.509 & 1 & .000 & .304 \\
\hline Between-subjects effects & \multicolumn{5}{|l|}{} \\
\hline Age & 2.030 & 1 & .162 & .048 \\
\hline Within-between interaction effects & 2.159 & 1 & .150 & .051 \\
\hline Mode*Age & 2.175 & 1 & .148 & .052 \\
\hline Amount*Age &
\end{tabular}

(b) Pairwise comparison for interactions effect between three within-subject factors 


\begin{tabular}{|c|c|c|c|c|c|c|c|c|}
\hline \multirow[b]{2}{*}{ Amount } & \multirow[b]{2}{*}{ Driving } & \multirow[b]{2}{*}{ (I) Mode } & \multirow{2}{*}{ (J) } & \multirow{2}{*}{$\begin{array}{c}\text { Mean } \\
\text { Difference } \\
\text { (I-J) }\end{array}$} & \multirow{2}{*}{$\begin{array}{l}\text { Std. } \\
\text { Error }\end{array}$} & \multirow[b]{2}{*}{ Sig. } & \multicolumn{2}{|c|}{$\begin{array}{c}\text { 95\% Confidence Interval for } \\
\text { Difference }\end{array}$} \\
\hline & & & & & & & Lower Bound & Upper Bound \\
\hline \multirow[t]{2}{*}{1} & 1 & 1 & 2 & .128 & .142 & .372 & -.158 & .414 \\
\hline & 2 & 1 & 2 & 1.137 & .109 & .000 & .916 & 1.358 \\
\hline \multirow[t]{2}{*}{2} & 1 & 1 & 2 & .633 & .126 & .000 & .378 & .889 \\
\hline & 2 & 1 & 2 & .241 & .119 & .049 & .001 & .481 \\
\hline \multirow[b]{2}{*}{ Mode } & \multirow[b]{2}{*}{ Driving } & \multirow{2}{*}{ (I) } & \multirow{2}{*}{ (J) } & \multirow{2}{*}{$\begin{array}{c}\text { Mean } \\
\text { Difference } \\
\text { (I-J) }\end{array}$} & \multirow{2}{*}{$\begin{array}{l}\text { Std. } \\
\text { Error }\end{array}$} & \multirow[b]{2}{*}{ Sig. } & \multicolumn{2}{|c|}{$\begin{array}{c}\text { 95\% Confidence Interval for } \\
\text { Difference }\end{array}$} \\
\hline & & & & & & & Lower Bound & Upper Bound \\
\hline \multirow[t]{2}{*}{1} & 1 & 1 & 2 & -1.419 & .137 & .000 & -1.695 & -1.142 \\
\hline & 2 & 1 & 2 & -.985 & .121 & .000 & -1.230 & -.741 \\
\hline \multirow[t]{2}{*}{2} & 1 & 1 & 2 & -.913 & .163 & .000 & -1.242 & -.584 \\
\hline & 2 & 1 & 2 & -1.881 & .139 & .000 & -2.163 & -1.600 \\
\hline \multirow[b]{2}{*}{ Mode } & \multirow{2}{*}{$\begin{array}{l}\text { Amoun } \\
t\end{array}$} & \multirow{2}{*}{$\begin{array}{l}\text { (I) } \\
\text { Driving }\end{array}$} & \multirow{2}{*}{$\begin{array}{l}(\mathbf{J}) \\
\text { Driving }\end{array}$} & \multirow{2}{*}{$\begin{array}{c}\text { Mean } \\
\text { Difference } \\
\text { (I-J) }\end{array}$} & \multirow{2}{*}{$\begin{array}{l}\text { Std. } \\
\text { Error }\end{array}$} & \multirow[b]{2}{*}{ Sig. } & \multicolumn{2}{|c|}{$\begin{array}{c}\text { 95\% Confidence Interval for } \\
\text { Difference }\end{array}$} \\
\hline & & & & & & & Lower Bound & Upper Bound \\
\hline \multirow[t]{2}{*}{1} & 1 & 1 & 2 & -.602 & .141 & .000 & -.887 & -.317 \\
\hline & 2 & 1 & 2 & -.169 & .113 & .142 & -.396 & .059 \\
\hline \multirow[t]{2}{*}{2} & 1 & 1 & 2 & .407 & .105 & .000 & .195 & .620 \\
\hline & 2 & 1 & 2 & -.561 & .097 & .000 & -.758 & -.365 \\
\hline
\end{tabular}

*Mode 1: DMS; Mode 2: VDMS; Amount 1: three Units of information; Amount 2: four units of information; Driving 1: Low complexity; Driving 2: High complexity.

The results reveal significant effects for all three main within-subject factors (Mode, Amount, and Driving), all two-way interaction effects, and three-way interaction effects, as shown in Table 4-3 (a). Subjects were sensitive to the changes in all three factors, but the effect of changes in one factor is dependent on the changes of other factors. Thus, we need to further examine the post-hoc comparisons between the factors. 
The first block of Table 4-3 (b) shows that the effect of Mode on perceived difficulty level is significant under all combinations of Amount and Driving except under the most simple conditions (low information context and driving complexity). This implies that VDMS can significantly reduce the perceived difficulty level for drivers except under simplest conditions, where drivers perceive the two modes as having a similar difficulty level. The effect of Amount, shown in the second block of Table 4-3 (b), indicates a significant effect of Amount under all conditions. This was expected since people may be more subjectively sensitive to difficulty caused by increased amount of information. Driving complexity results are similar to those seen

for the comprehension level, and are shown in the third block of Table 4-3 (b). Complex driving conditions generally are perceived as making message processing more difficult for drivers than the simple driving condition. The exception was the DMS with a high information amount, possibly because of effect of more information amount is larger than the effect of more complex driving condition, partially demonstrated by the value of Partial Eta Squared in Table 4-3 (a) (Amount=0.803, Driving=0.233).

The between-subject variable, Age, is significant, while the interaction effects between within-subject factors and within-between interaction effects are not statistically significant. It can be concluded that there are significant differences in the perceived difficulty level of processing and retaining messages between young and old drivers. However, trends are in the effect of Mode, Amount, and Driving are similar in both younger and older drivers.

\subsection{Summary}

This study conducted a driving simulator-based experiment to examine the effectiveness of VDMS. Table 4-4 summarizes key attributes of VDMS compared with DMS.

Table 4-4 Comparison of DMS vs. VDMS 


\begin{tabular}{|c|c|}
\hline Aspect & Comparison between DMS and VDMS \\
\hline Comprehension & $\begin{array}{l}\text { - For short messages and simple driving conditions, there was no significant } \\
\text { difference between DMS and VDMS. VDMS has a significantly higher } \\
\text { comprehension rate when information loads are high or when driving } \\
\text { conditions became more complex, however. } \\
\text { - VDMS can be used to provide longer messages than DMS under simple } \\
\text { driving conditions since comprehension is not negatively impacted by } \\
\text { message length for VDMS. }\end{array}$ \\
\hline Distraction & $\begin{array}{l}\text { - Time to brake with VDMS was on average } 0.39 \text { seconds shorter than DMS } \\
\text { under both simple and complex driving conditions } \\
\text { - Complex driving conditions significantly increased time to brake with the } \\
\text { DMS, while time to brake did not significantly increase with driving } \\
\text { complexity for VDMS. } \\
\text { - The above two comments apply to both young and old drivers }\end{array}$ \\
\hline Difficulty & $\begin{array}{l}\text { - VDMS had a significantly lower perceived difficulty level for drivers than } \\
\text { DMS except under simplest conditions (low information load and simple } \\
\text { driving condition) } \\
\text { - The above comment applies to both young and old drivers }\end{array}$ \\
\hline
\end{tabular}

Based on this evaluation, VDMS provides a means of providing traffic information that is comparable, and possibly better, than currently used DMSs. It can increase drivers' message comprehension level and can potentially deliver more information. Also, VDMS showed positive results in reducing time to brake versus DMS messages.

This chapter investigated the effectiveness of using in-vehicle auditory systems, VDMS, to deliver public traffic messages as compared with DMS. The findings revealed that VDMS is generally a better way to deliver traffic messages of different amounts of information and under 
different driving conditions for both young and old participants. VDMS can generally help increase message comprehension, reduce distraction, and make the same messages easier to process and retain messages for drivers than DMS. Also, drivers potentially prefer VDMS to DMS to receive information. The conclusion of this paper revealed the advantages of VDMS and can serve as evidence for public agencies to deploy VDMS systems in the future.

Since this was the first study to evaluate the feasibility and effectiveness of VDMS, there are still some limitations and unanswered questions to be explored in the future. First, although driving simulators are commonly used in traffic research due to their practicality and high level of experimental control, replications of the study in real-life driving settings, such as via on-road assessments, are needed in order to ensure the generalizability of the findings. Second, the current experimental design is simplified in some aspects (e.g., two levels for each factor), and ignores some other factors (e.g., gender, education level). Further studies are needed to investigate more factors with more levels, such as higher levels of information. Third, the VDMS auditory message in this paper are only audio version of similar visual DMS messages, while in the practice there are other strategies to make VDMS messages more effective, such as repeating key and difficult information twice, which is not achievable in DMS. The effectiveness of these improvements should be further evaluated as well to improve the VDMS design. Also, displaying more complex messages (e.g., maps/visual texts on an in-vehicle device screen along with detailed auditory messages) are not considered in this paper. Further studies can be conducted whether it is appropriate for DOTs to provide detailed information while limiting the distractions to reasonable level.

Next, there are other human factor issues for VDMS messages, such as characteristics of the voice and structure of the auditory messages, are issues that need to be addressed in future research. Another possible future item would be to examine levels of ambient noise and their effect on driver performance with VDMS. Last, this study used scenarios on a local network that 
subjects would be familiar with. It is important to investigate whether results hold for non-local drivers traveling an unfamiliar network. 


\section{CHAPTER 5 AN APPLICATION: ROUTING}

\section{ADVISOTIES FOR EMERGENCY TRAFFIC}

\section{MANAEMENT}

After the effectiveness of VDMS is confirmed by Chapter 4, along with its capability of scalability and personalization, VDMS can be a powerful traffic management tool itself by better providing travelers with real-time information. Also, VDMS has the potentials to enable more advanced traffic management strategies, such as emergency routing, speed advisory for speed harmonization, and lane change advisory for merge control. This chapter selects and evaluates an advanced emergency routing strategy, among numerous possible VDMS applications, to further demonstrates the potentials of VDMS in traffic management.

\subsection{Introduction}

Under emergencies, including major incidents, severe weather conditions or hazmat spill, the ratio between demand and capacity will greatly exceed one and it is necessary to have sophisticated approach to optimally utilize capacities of existing facilities, and maintain/restore traffic movement. Publishing area-wide or corridor-level information through DMS can be helpful, but providing routing advisories for best use of facilities under emergencies is quite 
meaningful to prevent severe congestion and help travelers arrive at destinations as early as possible.

The proposed concept of VDMS has been proven effective in delivering more information in a scalable manner with low driver distraction. It also has the ability to provide information personalized to each driver instead of one-for-all provision. These advantages can not only greatly help transportation system operators reduce the cost and complexity of maintaining information infrastructure but also effectively use bi-directional communications to manage vehicular networks.

This chapter proposes the idea of Personalized System Optimum Information (PSOI) provision for traffic management under emergencies and aims to develop models and algorithm to generate optimal routing advisories. We are interested in evaluating the amount of benefits such flexible information provision strategy can lead to.

\subsection{Methodological Contributions}

In terms of modeling and simulation, the methodology adopted in this study differs from many in the literature, either on VMS-type route guidance or route guidance from a private-sector service provider. Table 1 is used to demonstrate the difference of this paper and many other most relevant papers in the literature from six aspects.

Table 1 Comparison of Models from Different Key Aspects for Proposed Approach

\begin{tabular}{|l|l|l|}
\hline $\begin{array}{l}\text { 1. Agent(individual)/Flow- } \\
\text { based Modeling }\end{array}$ & $\begin{array}{l}\text { Agent/individual-based: } \\
\text { This paper; Lee \& Park } \\
\text { (2008); Chen et al. (2014); } \\
\text { Osorio \& Bierlaire (2010); }\end{array}$ & $\begin{array}{l}\text { OD/Path Flow-based: } \\
\text { Shen et al. (2006); Paz \& Peeta } \\
(2007) ; \text { Peeta et al (2001); } \\
\text { Huynh et al. (2003); Chiu et al. } \\
\text { (2007); Howell \& Fu (2006); } \\
\text { Gao \& Huang (2012); } \\
\text { Ziliaskopoulos (2000); }\end{array}$ \\
& $\begin{array}{l}\text { Single-class: } \\
\text { Shen et al. (2006); Huynh et al. }\end{array}$ \\
\hline $\begin{array}{l}\text { 2. Mixed Users } \\
\text { (User/System Oriented) }\end{array}$ & $\begin{array}{l}\text { Mixed Users: } \\
\text { This paper; }\end{array}$ & \multicolumn{2}{|l}{} \\
\hline
\end{tabular}




\begin{tabular}{|c|c|c|}
\hline & & $\begin{array}{l}\text { (2003); Chiu et al. (2007); } \\
\text { Osorio \& Bierlaire (2010); Gao } \\
\text { \& Huang (2012); } \\
\text { Ziliaskopoulos (2000); } \\
\text { Multi-class: } \\
\text { Lee \& Park (2008); Chen et al. } \\
\text { (2014); Paz \& Peeta (2007); } \\
\text { Peeta et al (2001); }\end{array}$ \\
\hline 3. Purpose & $\begin{array}{l}\text { Traffic Management: } \\
\text { This paper; Chen et al. (2014); } \\
\text { Huynh et al. (2003); Peeta et al } \\
\text { (2001); Chiu et al. (2007); } \\
\text { Osorio \& Bierlaire (2010); }\end{array}$ & $\begin{array}{l}\text { Navigation: } \\
\text { Lee \& Park (2008); Paz \& Peeta } \\
\text { (2007); Gao \& Huang (2012); } \\
\text { Ziliaskopoulos (2000); }\end{array}$ \\
\hline 4. Predictability & $\begin{array}{l}\text { Consider experienced traffic } \\
\text { state: } \\
\text { This paper; Shen et al. (2006); } \\
\text { Lee \& Park (2008); Chen et al. } \\
\text { (2014); Huynh et al. (2003); } \\
\text { Chiu et al. (2007); Howell \& } \\
\text { Fu (2006); Osorio \& Bierlaire } \\
\text { (2010); Gao \& Huang (2012); } \\
\text { Ziliaskopoulos (2000); }\end{array}$ & $\begin{array}{l}\text { Use instantaneous traffic } \\
\text { state: } \\
\text { Lee \& Park (2008); Paz \& Peeta } \\
\text { (2007); Peeta et al (2001); }\end{array}$ \\
\hline 5. Optimization & $\begin{array}{l}\text { Simulation Optimization } \\
\text { (e.g., Gradient-based): } \\
\text { This paper; Shen et al. (2006); } \\
\text { Chen et al. (2014); Howell \& } \\
\text { Fu (2006); Osorio \& Bierlaire } \\
\text { (2010) } \\
\text { Rolling Horizon: } \\
\text { Paz \& Peeta (2007); Peeta et al } \\
\text { (2001); }\end{array}$ & $\begin{array}{l}\text { (Meta-) heuristics: } \\
\text { Huynh et al. (2003); Chiu et al. } \\
\text { (2007); Gao \& Huang (2012); } \\
\text { Traditional flow-based } \\
\text { methods (e.g., MSA): } \\
\text { Shen et al. (2006); } \\
\text { Mathematical Programming } \\
\text { Method: } \\
\text { Ziliaskopoulos (2000); }\end{array}$ \\
\hline $\begin{array}{l}\text { 6. Computational Challenge } \\
\text { (large network and real time } \\
\text { deployment) }\end{array}$ & $\begin{array}{l}\text { Efficient: } \\
\text { This paper; Huynh et al. } \\
(2003)\end{array}$ & $\begin{array}{l}\text { Limited: } \\
\text { Shen et al. (2006); Lee \& Park } \\
\text { (2008); Chen et al. (2014); } \\
\text { Peeta et al (2001); Paz \& Peeta } \\
\text { (2007); Chiu et al. (2007); } \\
\text { Howell \& Fu (2006); Osorio \& } \\
\text { Bierlaire (2010); Gao \& Huang } \\
\text { (2012); Ziliaskopoulos (2000); }\end{array}$ \\
\hline
\end{tabular}

Based on Table 1, the contributions of this paper are better illustrated as the following bullets:

\section{Individual-based Modeling}


This paper combined individual-based modeling and optimization approach for dynamic traffic management. Compared with traditional OD/path flow-based models, the adopted approach has the following advantages:

- Ability to track each individual vehicle: As shown in Figure 5-1 Comparison between Flowbased Modeling and Individual-based Modeling, traditional flow-based approach tracks vehicle flow using origins, destinations and departure time. However, due to factors like traffic congestion, vehicles in the same flow may reach different locations at the same time. Flow-based models are not able to describe these attributes. In the meantime, individual-based models can track the locations of each vehicle (e.g.,) and separately consider them in the modeling in a more targeted way. Most importantly, real-time data can be incorporated easily into such models, such as GPS locations.

- Ability to model each individual's behavior: Different vehicles in the same flow may have different attributes and different behavior. Individual-based modeling is able to assign each agent with different attributes (current location, destination, VOT, historical routes, etc.), and thus let them make different decisions even when they belong to the same flow.

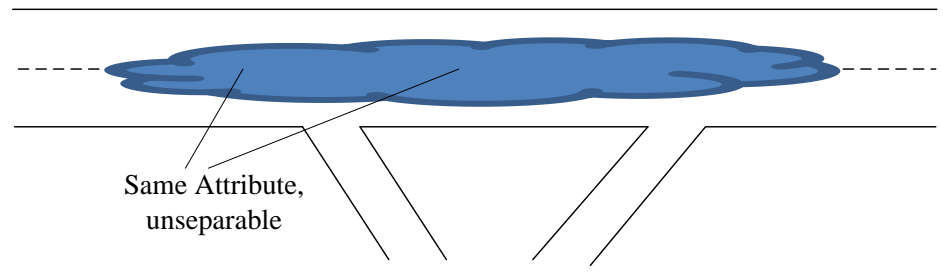

Flow-based Modeling

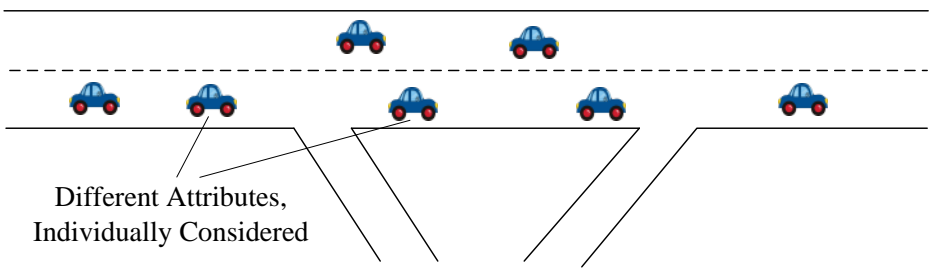

Agent-based Modeling 
Figure 5-1 Comparison between Flow-based Modeling and Individual-based Modeling

\section{Optimization for Traffic Management (Personalized SO)}

The information strategy developed in this paper is for dynamic traffic management, instead of personal route choice, and thus needs optimization as a key component of the computation process.

Combined with Individual-based modeling, this paper is a Personalized System Optimum (SO) problem, which is very different from traditional flow-based SO. Other than differences discussed in Bullet 1, interesting results are also obtained from analysis results of this paper, such as Personalized SO users experience travel times that are at least the same as other types of users. This makes Personalized SO also a nice algorithm that makes PSOI also serve as a personal decision tool and more accepted to travelers.

\section{Solution Approach}

For the simulation-based heuristic algorithm, this paper developed a simple and efficient queue based heuristics for optimization. Queue is evaluated at each decision time point and link marginal time calculated by adding queue delay. The search direction for each iteration is decided by letting some travelers choose paths with shortest marginal delay. In order to avoid system performance oscillation, the algorithm uses a heuristic small step size in each iteration, by selecting a small proportion (5\%) of travelers for new route searching.

Also, there are great needs for providing personalized traffic information in real time, so there is a computational efficiency requirement. As demonstrated by our case studies, the proposed algorithm is efficient in finding near-optimal solutions even for large networks and thus truly applicable for real time traffic management. 
The advantages using simple queue based framework with queue-based network propagation modeling and the nice use of simple queue based heuristics, is also demonstrated using our case studies. Without using our model, the literature usually use a large number of simulation runs for flow perturbations. Some studies use meta-heuristic like GA for obtaining new solutions, involving many runs in each iteration, is computationally intractable for large networks and real time implementation.

\section{Predictability with Mixed Travelers}

The concept of mixed agents/individuals in this paper is different from multi-class agents. Traditional multi-class models consider travelers with different behavior (such as shortest path, bounded rationality) or travelers that can receive different types of information (but with the same objective like User-oriented). This paper considers the mixed user environment today, where users may use their historical knowledge, user-oriented pre-trip/en-route information, or information provided by TMC for traffic management (as the information strategy of this paper).

Also, the Personalized SO strategy in this paper is a predictive strategy, which means the optimization should be based on each traveler's experienced travel time, rather than instantaneous travel time at decision point. The behavior of other users may complicate this problem, since their route choice is also time dependent and dependent on the choices of other travelers. The iterative optimization algorithm successfully solves this issue by cautiously find better solutions in each iteration using a small step size, maintaining generally descent direction from iteration to iteration.

This paper focuses on developing and evaluating the potentials of PSOI, and thus makes a few assumptions to facilitate the model development:

- Assume that the changes in roadway capacity and duration are known: While these factors are also important, it is not this paper's focus to develop predictive models for these input variables or other forms of modeling to account for the uncertainty. 
- Assume full compliance with PSOI and other information strategies: while compliance rate is generally an important issue, we focus on how the strategy can be efficiently devised and knowing the upper bound - the optimal system performance that such a strategy can bring. Thus, we made the assumption that all travelers who opt in such program are happy to comply. Also, compliance rate is not an important question for such a strategy, due to the robustness of proposed algorithm. If some travelers will not comply, as long as there are other PSOI users, the algorithm will eventually select enough travelers to optimize the system to the same level. If there are not enough PSOI users, the system performance will then bounded by market penetration, not compliance rate. Thus, market penetration is a more important factor in this paper.

\subsection{Simulation Based Heuristics Approach}

\subsubsection{Individual-based Simulation Model}

A simulation-based approach is adopted in this paper for developing and evaluating a VDMSenabled advanced routing advisories under emergencies. Among different levels of simulation, the mesoscopic simulation method, usually referred to as simulation-based dynamic traffic assignment (DTA) models, is considered the best fit for the purpose of this study. It is because that DTA models are able to address larger transportation networks than microscopic simulation but also simulate individual vehicles' movement and choice dynamically during each vehicle's trip.

There are many simulation-based DTA models/tools available for this study purpose, such as DYNASMART (Jayakrishnan et al., 1994), DTALite (Zhou \& Taylor, 2014), DynaMIT (Ben-Akiva, 2012) and DynusT (Chiu et al., 2008). Although these models simulate the movement of vehicles individually, many of its applications (e.g., Lu et al., 2008) are still using 
flow-based frameworks and these tools are used for dynamic network loading. This paper proposes an individual-based framework, modeling the travel behavior of each individual considering their characteristics, such as the personal value of time, allowable budgets for congestion tolling, and willingness to taking detours. Specifically, the algorithm proposed in this paper also handles individual vehicles instead of flows when searching for traffic flow assignment for the next iteration. By considering individuals throughout the computation framework, we aim to build a true individual-based framework where individuals behave or are controlled individually and traffic flow is only reflected by interaction of agents with each other.

\subsubsection{Personalized System Optimum Traveler Information (PSOI)}

The emergency routing strategy PSOI in this paper includes three important components that are considered as the key to effective traveler information systems in this paper: "Personalization", "Predictability", and "System Optimum (SO)". "Personalization" corresponds to the individual-based modeling approach we adopt. In the simulation framework of this paper, each vehicle is modeled individually with different characteristics. We consider that each individual is unique and different from other individuals in terms of various attributes in the simulation at a certain time: location, origin, destination, habitual routes, and value-of-time, allowable budgets for congestion tolling, and willingness to taking detours, etc. Unlike flowbased models, the agent-based model calculates the best information strategy for each individual.

"SO" is the concept that is compared with UO. In a SO strategy, each individual will not be short-sighted and pick the current shortest path. Instead, vehicles will use the routes that potentially help optimize total system performance. This paper uses the concept of link marginal delay from Ghali \& Smith (1995), represented visually in Figure 5-2, where the vehicle arrival curve $A(t)$ and vehicle departure curve $\mathrm{D}(\mathrm{t})$ is the traffic condition at the end of a link, and

insufficient downstream link capacity makes this point a bottleneck. At time ${ }^{t_{1}}$, when the vehicle 
arrival rate is greater than the departure rate, a queue forms. When a single vehicle (one unit of

flow) arrives at time ${ }^{t_{2}}$, the queue length is $q$, the vehicle will be able to depart at time ${ }^{t_{2}}$ and the total link delay the vehicle would encounter is $d=t_{3}-t_{2}$. However, the arrival of this vehicle also caused delay for other vehicles arriving here from time ${ }^{t_{2}}$ to ${ }^{t_{4}}$ (queue dissipation time), and the additional delay imposed on others is $t_{4}-t_{3}$. Thus, the SO link travel time of a link is the summation of actual link travel time and marginal delay. Based on this theory, by assigning selected shortest $\mathrm{SO}$ routes to vehicles, we can achieve the best system performance.

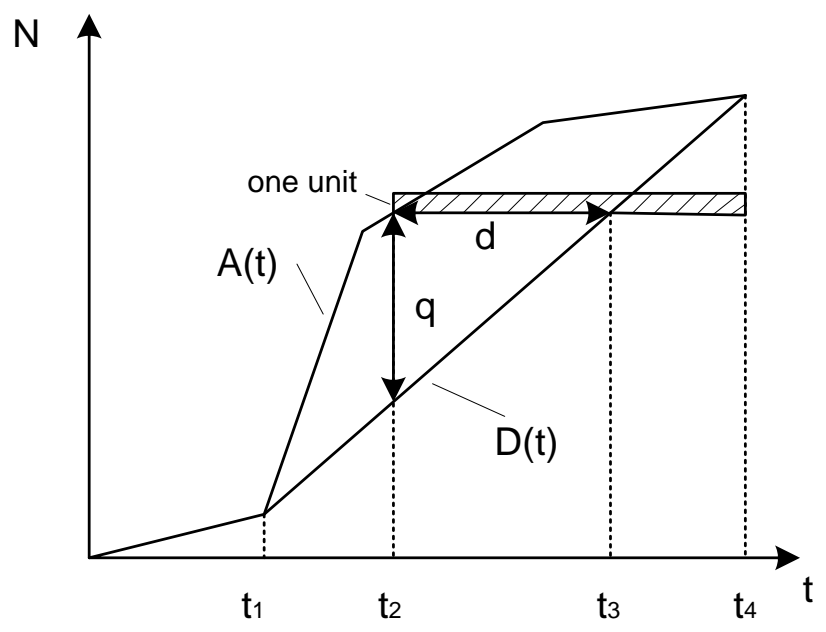

Figure 5-2 Illustration of the concepts of link and path marginal delay

Based on Bellman's principle of optimality, some modified label correcting algorithms (Ziliaskopoulos \& Mahmassani, 1992) can be adopted to search for the optimal time-dependent shortest SO route. In the searching procedure, an M+1-dimensional vector-based label, denoted by $\Gamma_{j}=\left(\eta_{j}\left(t_{0}\right), \eta_{j}\left(t_{0}+\sigma\right), \cdots, \eta_{j}\left(t_{0}+\mathrm{M} \sigma\right)\right)$, can be used to show the least travel cost from 
origin $\mathrm{O}$ to current node $\mathrm{j}$ at each time stamp $t^{\prime} \in T$, where $\eta_{j}\left(t^{\prime}\right)$ is computed by $t^{\prime}=t+c_{i j t}$ : $\eta_{j}\left(t^{\prime}\right)=\left\{\begin{array}{cr}\min \left\{\eta_{j}\left(t^{\prime}\right), g_{i j t}+\eta_{j}(t)\right\}, & j \in N \backslash i, t^{\prime} \in T \\ 0 \quad, j \in O, t^{\prime} \in T\end{array}\right.$

where $c_{i j t}$ is the time-dependent travel time of link (i,j) at time t, and $g_{i j t}$ is the generalized time-dependent link cost including marginal delay for the SO route.

The real-time nature of the problem of this paper requires high computational efficiency. Thus, simple queue-based dynamic network loading models are used in this paper, such as point queue, spatial queue, (Zhang et al., 2013) or Newell's Simplified Kinetic Wave model (Yang \& Zhou, 2014; Zhou \& Taylor 2014). Among them, Newell's Simplified Kinetic Wave is used in this paper since its proven accuracy and simple structure using arriving and departing curves as in Figure 1. Constrained by outgoing and incoming link capacity, the number of vehicles in the queue of each link can be easily evaluated and then be used to evaluate link marginal cost. Readers can refer to the literature for detailed introduction to this theory (Newell, 1993).

"Predictability" indicates that the travel time estimation for each vehicle is the actual travel time to be experienced by the vehicles, instead of instantaneous travel time. In this paper, this is achieved through simulation by running multiple iterations of route assignment to all vehicles until the stop criteria is met (e.g., convergence is reached or maximum number of iterations is reached). To be more specific, in each iteration, a proportion of vehicles will be assigned with best SO routes individually based on the current instantaneous network state, such as link travel time and queue length. Afterwards, a dynamic network loading model is run to obtain the predicted network state if the route advisory of this iteration is used. Since we are considering each vehicle in calculating the shortest path, we may encounter significant system performance oscillation and deterioration if we divert all vehicles which can find a route with 
lower SO generalized cost. It is necessary to ensure the descent direction and prevent oscillation to avoid over-diverting vehicles in each iteration. A heuristic approach is used in this paper - only a small percentage of agents will be assigned $\mathrm{SO}$ routes in each iteration.

\subsubsection{Optimization}

From the perspective of optimization, the heuristic steps for last two attributes "SO" and "Predictability" represent two important concepts in optimization theory: search direction and step size. In each iteration, we use link queue information based on Newell's simplified Kinetic Wave theory to calculate link marginal delay, in order to find shortest path with marginal path delay. This process is similar to the descent direction search in each direction. Since the sum of link marginal delay is not necessarily equivalent to path marginal delay, as mentioned by Ghali and Smith (1995), the direction obtained through the method is not strictly descent. Thus, we use a small step size in each iteration - only rerouting a small percentage of randomly selected travelers - in order to avoid system performance fluctuation. Although this method cannot guarantee a strict descent direction and may only obtain near-optimal solutions, the simple method works well and is highly efficient, as demonstrated by our case studies.

Combining the requirements of all three components, we developed a simple and efficient queue based heuristic algorithm to search for an optimal information strategy. Queue is evaluated at each decision time point and link marginal time calculated by adding queue delay. As illustrated in Figure 2, the complete solution procedure is as follows. Note that while some literature also addressed similar information type and proposed analytical models and algorithm for optimal solution (Ma et al., 2014), this paper instead proposes an easy-to-use and practical for use by Transportation Management Centers, particularly for routing advisories under emergencies.

PSOI Algorithm: 
Step 1: Obtain characteristics of each vehicle/individual such as historical routes, real-time location, and value-of-time, etc., as algorithm input; Set iteration number $\mathrm{n}=0$;

Step 2: Randomly select $x \%$ (a small percentage, e.g., 5\%) of all vehicles to change routes to SO routes for this iteration.

Step 3: For each vehicle selected in this iteration, determine the vehicle's new route with smallest marginal delay, calculated by summing the local marginal delay of each link on the route. If a new route is found, divert the agent to this new route. If not, this agent stays on the original route.

Step 4: Run simulation (dynamic network loading) to obtain total system travel time (TT). If the percent changes of TT compared with last iteration is smaller than a certain value $T T^{(n)}-T T^{(n-1)}<\varepsilon_{\text {or }}$ iteration counter reaches the pre-set maximum number $n>N_{\max }$, go to Step 5. Otherwise, $n=n+1$ and return to Step 2 .

Step 5: Output the route assignment of the last iteration as the final PSOI information strategy. 


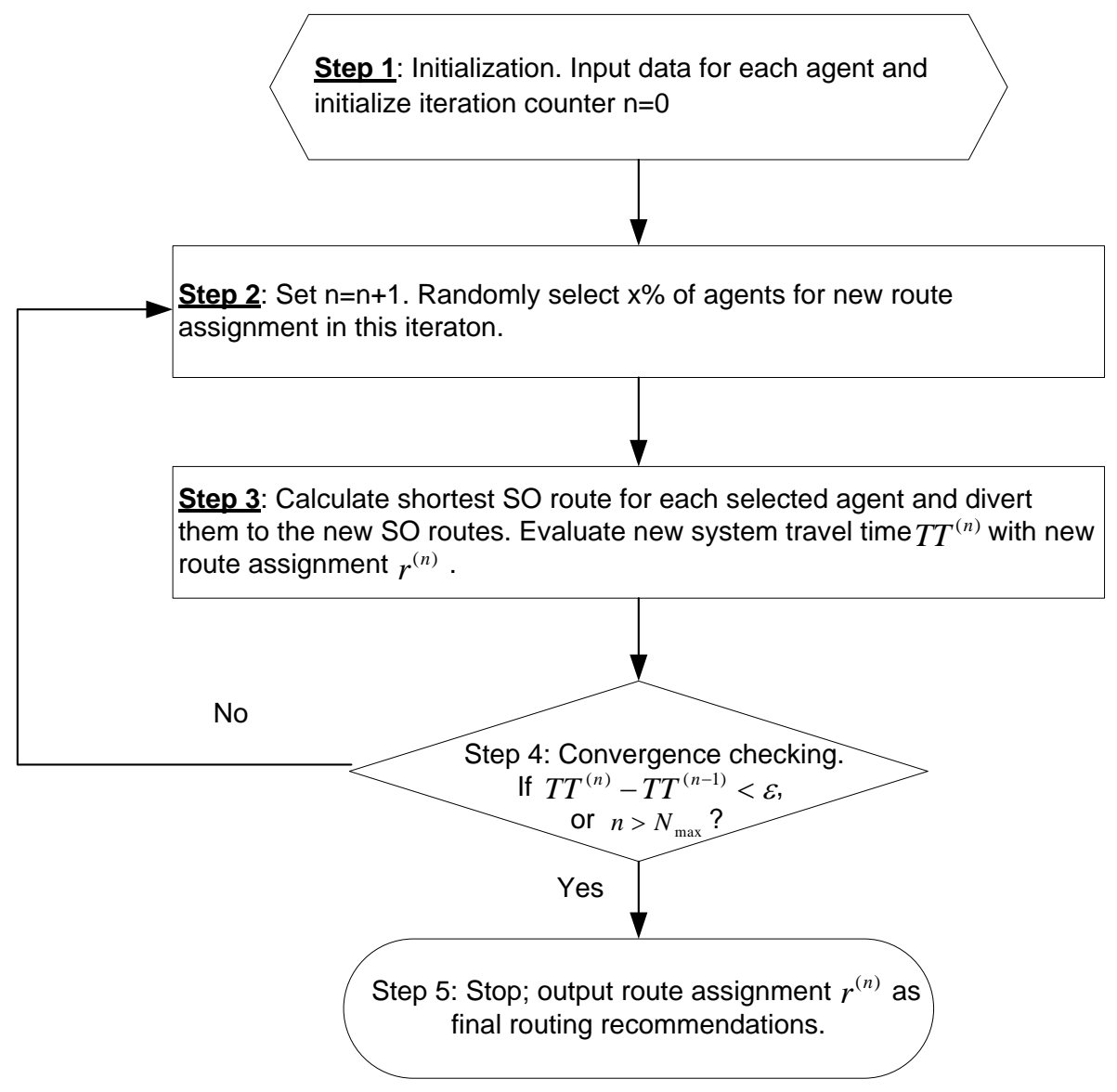

Figure 5-3 PSOI algorithm for Personalized SO Information Strategy

The advantages of using simple queue based framework with queue-based network propagation modeling and the nice use of simple queue based heuristics, is also demonstrated using our case studies. Without using our model, the literature usually use a large number of simulation runs for flow perturbations. Some studies use meta-heuristic like GA for obtaining new solutions, involving many runs in each iteration, is computationally intractable for large networks and real time implementation. (e.g., Howell, 2006; Chiu et al., 2007)

The PSOI algorithm is proposed based on the assumptions that some vehicles are connected vehicles (two-way information exchange) and all agent are happy to comply such personalized information if they belong to the group (opt-in). The purpose of this paper is to 
consider the potential benefits of PSOI for emergency routing. We focus on how the strategy can be efficiently devised, and want to know an upper bound - the optimal system performance that such a strategy can bring. Thus, we made the assumption that all travelers who opt in such program are happy to comply. However, these assumptions can be easily relaxed by using algorithms adapted from the proposed one. For example, considering less than $100 \%$ full compliance, if personal device could learn the compliance rate of travelers from previous days, "compliance" can be another agent attribute and incorporate directly to the algorithm with addition of only Monte Carlo simulation to decide compliance before calculating shortest SO path. Eventually, we will be able to provide detailed information such as diversion to those who are willing to divert and who are at critical routes with median or high diversion rates.

Also, compliance rate is not an important question for such a strategy, due to the robustness of proposed algorithm. If some travelers will not comply, as long as there are other PSOI users, the algorithm will eventually select enough travelers to optimize the system to the same level. If there are not enough PSOI users, the system performance will then bounded by market penetration, not compliance rate. Thus, market penetration is a more important factor in this paper.

\subsubsection{Other Information Classes and Strategies}

In today's transportation network, different information providers coexist. Public agencies provide network state information using VMS and private sectors provide routing information using personal devices. Thus, a new information strategy, emergency routing advisory in this paper, needs to be effective when other information strategies co-exist. In this paper, in addition to PSOI, we consider four other strategies that are commonly used in real world applications: historical information (HI), pre-trip information (PTI), en-route information (ERI), and variable message signs (VMS). The concept of mixed agents in this paper is different from 
multi-class agents. Traditional multi-class models consider travelers with different behavior (such as shortest path, bounded rationality) or travelers that can receive different types of information (but with the same objective like User-Optimal). This paper considers the mixed user environment today, where users may use their historical knowledge, user-oriented pre-trip/enroute information, or information provided by TMC for traffic management (as PSOI of this paper).

Also, the Personalized SO strategy in this paper is a predictive strategy, which means the optimization should be based on each traveler's experienced travel time, rather than instantaneous travel time at decision point. The behavior of other users may complicate this problem, since their route choice is also time dependent. The iterative optimization algorithm solves this issue by cautiously find better solutions in each iteration using a small step size, maintaining generally descent direction from iteration to iteration.

This sub-section will address their definitions in this paper and detail the modeling approach in the same agent-based simulation framework. Also, it is important to be able to model different types of information strategies under the same framework since drivers traveling on real networks may use different information groups.

\section{(1) Historical Information (HI)}

People which travel daily on the network without any extra information are part of the historical information (HI) group. These people are familiar with the network and have fixed, chosen routes after a period of day-to-day adjustments. These chosen routes are usually the shortest path given daily stable network traffic. The HI group is considered as the base case condition in this paper.

In this study, we simulate the choice process by running the base case network without any incidents for multiple iterations (simulating multiple days) until network stability. Note that 
in the simulation, travel time estimates used to compare routes between an Origin and Destination in the route choice process are based on the average experienced travel time from trips with the same departure time interval in prior simulation iterations (i.e. previous day in a day-to-day learning environment). The travelers select paths which minimize their travel time based upon these travel time estimates. After obtaining network stability, we record each individual traveler's route from the last iteration/day as the historically chosen route to be used later as the basis for modeling other information strategies.

\section{(2) Pre-trip Information (PTI)}

Travelers which obtain information before their trip through multiple channels, such as Internet and smartphone apps, are called the Pre-trip Information (PTI) group. This information helps travelers to update their network knowledge of current travel times and make better route choice decisions. Travelers usually check PTI before their trip and thus only learn the instantaneous network state at the start of the trips. The same concept is used in this paper.

In our simulation, travel time estimates used to compare routes between an Origin and Destination in the route choice process are based upon current travel time data. In this case, the current path travel time estimates are based upon link travel times experienced by other drivers at the traveler's departure time. Again, the traveler selects a path which minimizes their travel time based upon these travel time estimates.

(3) En-route Information (ERI)

Travelers can also obtain information during their trip using advanced tools, such as GPS guidance systems with real-time traffic data. These systems usually retrieve information from a central server on a certain time interval basis and calculate/update the shortest path for the travelers. These systems are usually only accessible to the single user and have no access to other travelers' data. 
In our simulation, travel time estimates used to compare routes between an Origin and Destination in the route choice process are based upon current travel time data. In this case, the current path travel time estimates are based upon link travel times experienced by other drivers over multiple time intervals (e.g. in the last $15 \mathrm{~min}$ ) during the trip, with link travel times associated with the interval in which the vehicle would enter each link for different paths. The agent/driver selects a path which minimizes their travel time based upon these travel time estimates, but they also have the option to change their path each minute during the simulation. Travel time information is provided at the end of a link. Specifically, link travel times are updated at specific travel time aggregation time intervals (e.g., 5 minutes), and travel time estimates for different information classes incorporate randomized error (driver perception error).

\section{(4) Variable Message Signs (VMS)}

In this paper, VMSs are deployed at limited locations to cause travelers to consider changing their current route. A VMS is located on a link in the network, and vehicles passing through the link are potentially affected by the sign. Each VMS has a response rate that can be specified as an input, representing the probabilistic percentage of travelers which are affected by the sign and decide to consider a new route to their destination. If an agent considers selecting a new route, a new shortest path calculation is performed from the end of the link (on which the VMS is located) to the traveler's destination. It is assumed that the VMS provides some travel time information (based on prevailing travel condition) to the driver to help them select a new route, which is incorporated into the shortest path calculation. In the simulation, this travel time information is updated at a specified time interval (e.g., 30 minutes).

\subsubsection{Implementation in a Mesoscopic DTA}

Mesoscopic DTA models are chosen as the simulation engine for this paper since it is an agentbased dynamic traffic assignment model and supports most travel information provision services 
in its route choice model, utilizing simple, flexible user inputs to assign information classifications (or information classes) to specific agents while maintaining computational efficiency. The proposed model and algorithm can be applied or tested in any mesoscopic DTA tools but we select open-source DTALite for a quick demonstration using a data hub to bridge the model and algorithm with many data sources. A few studies have successfully used it as the engine for prediction and evaluation (Yang \& Zhou, 2014; Jia et al., 2011).

In DTALite, an agent or driver chooses a route based on the generalized cost to travel between an origin and destination. This generalized user cost is based upon three components: travel time, value of time (VOT), and tolling/pricing: Cost $=$ Travel Time $\times$ VOT + Toll. Each variable in the generalized cost equation is specific to the agent, corresponding to the key concept of personalization. Every agent has their own value of time, and travel times through the network are estimates which are dependent upon the type of information strategies available to that agent. Agents following the PSOI strategy are assigned to SO routes, while other agents are assumed to select the route which minimizes their own cost (Wardrop's 1st Principle) when offered multiple routes with different estimated travel costs. When making successive decisions about route choice (e.g., choosing a new path after departure, and/or choosing a path in the second simulation iteration), the new path must overcome the "willingness to switch" paths, which is a relative threshold used when comparing travel times. That is, the agent may decline to change paths if the alternative path does not meet some minimum travel time improvement (i.e., $5 \%$ travel time savings).

Groups of agents/drivers are randomly assigned to different information classes in DTALite based on percent distributions. For example, approximately $10 \%$ of drivers may use pre-trip information to choose a route to their destination, another $10 \%$ might use en-route information through a GPS-enabled device (and/or other service) to choose their route, and the remaining population might use historical information. Since this distribution is utilized when 
generating agents in the network, it is considered a demand attribute, and it is applied separately to different demand types. The user can define a demand type (e.g., SOV, HOV, Truck, etc.), and associate a specific information type distribution for each demand type.

\subsection{Case Studies}

Two case studies are conducted in this section. First, a three-corridor network is used for conducting sensitivity analysis to derive more insights into the PSOI strategy compared with other information strategies. Second, a real world subarea network is used to test the performance of the proposed PSOI algorithm.

\section{Three-corridor Network}

The first set of numerical experiments is conducted on a three-corridor network, which includes 40 nodes, 55 links and 2 OD zones (depicted using dashed lines) shown in Figure 5-4. In the base case scenario, a total of 7200 vehicles need to be evacuated from zone 1 to zone 2 (from left-most node to right-most node) during the simulation time (7AM-9AM) as quickly as possible. We run 20 simulation iterations to simulate 20 days of day-to-day route choice adjustments. The final result is that all vehicles choose the upper corridor (Figure 5-4) as their habitual path. The average travel time is $11.1 \mathrm{~min}$. In the incident scenario, an incident occurs at 7:30AM - 8:20AM (450 $\mathrm{min}-500 \mathrm{~min}$ ), reducing the capacity of link $9-10$ by $60 \%$ (location is indicated by red symbol in Figure 5-4). For historical information users who have no access to any information, they will still follow their historical routes, which is the upper corridor. 


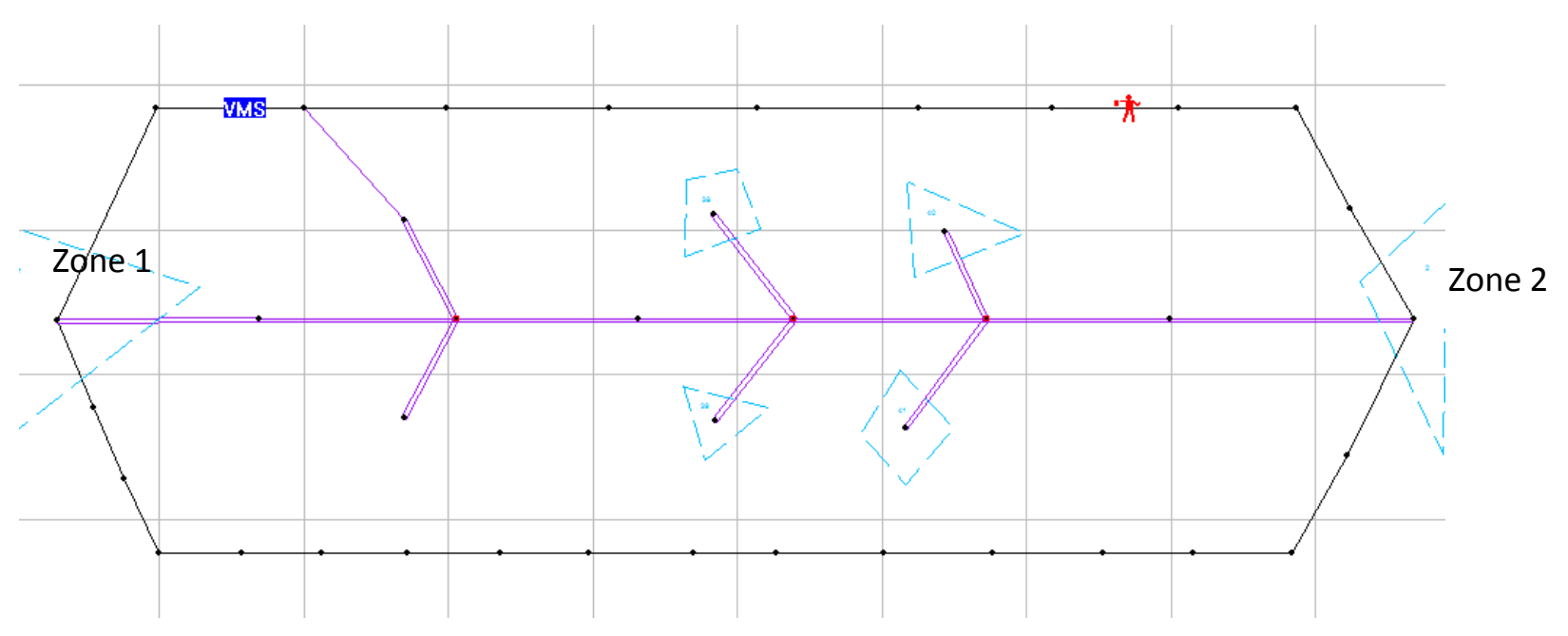

(a) Three-corridor network

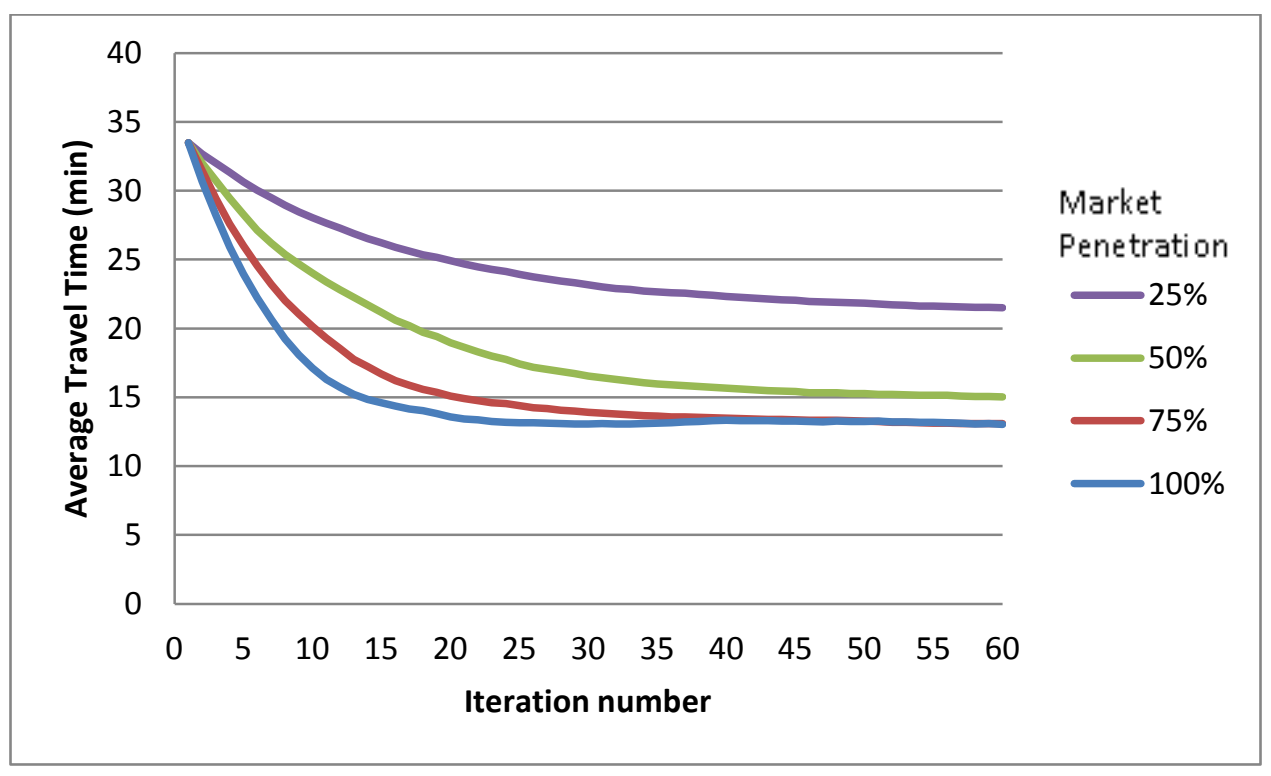

(b) Algorithm performance under different penetration rate

Figure 5-4 Algorithm Performance on Three-corridor Test Network.

We first examine the performance of the PSOI algorithm under different SO information market penetration rates. In each iteration, $5 \%$ of vehicles are selected randomly to be assigned the SO routes. Figure 5-4 (b) shows the algorithm performance over 60 iterations. If we set the 
algorithm stop criteria of $1 \%(0.5 \%)$ difference in average travel time between two consecutive iterations, the algorithm will stop at iteration $17,27,22$, and $21(33,35,29,24)$ for $20 \%, 50 \%, 75 \%$ and $100 \%$ PSOI market penetration, respectively. For this network, the average CPU run time on a PC with $4 \mathrm{~GB}$ memory and dual core processors running at $2.70 \mathrm{GHz}$ is about 2.82 seconds. Also, the experiments show that the algorithm maintained a descent direction in the first 20+ iterations when the consecutive difference in average travel time is still large. After that, the algorithm only ascends occasionally with very small ascent margins. As we can see, this algorithm is effective in searching for the near-optimal solution of routes of each individual for traffic system management.

Next, we can also derive some attribute of PSOI provision for the system performance from Figure 5-4 and Table 5-1 (a). As expected, a larger penetration rate may lead to better system performance. Note that, theoretically, a $100 \%$ penetration rate will result in best system performance, as shown in the high demand scenario (10800) in Table 5-1 (a). However, the low demand scenario (7200) shows a slightly better result of $75 \%$ penetration than $100 \%$. This may be because of the heuristic nature of the algorithm and it only found the near-optimal solution for the $100 \%$ case at the end of the 60th iteration. But the actual difference between the optimal value of $75 \%$ and $100 \%$ cases may be very small, as indicated by both demand scenarios. From the perspective of traffic management, it implies that when an incident occurs, it is not necessary to provide the SO information to all drivers, the improvement of system performance will be only marginal after a relatively large penetration, such as $75 \%$.

An interesting result from Table 5-1 (a) is that, while the total system performance (measured by average travel time) is improved as the penetration rate increases, the average travel time of SO travelers increases. This is reasonable since under a low penetration rate fewer travelers use the other two corridors and thus SO travelers who use these uncongested corridors can experience shorter travel times. But still, under different penetration rates, the SO traveler 
always experiences less or similar travel time than other HI travelers. This implies that PSOI can also help individual users to pick quicker routes, not just improving the system. Note that the average travel time of SO is slightly higher than $\mathrm{HI}$ users for $75 \%$ penetration rate under low demand. This is because that part of the SO travelers diverted a little to make sure HI travelers can experience short travel times. The largest SO traveler travel time can be marginally larger than $\mathrm{HI}$ users because SO users would choose HI routes if travel time difference of HI route and other routes is significant. Although it seems that SO users are traveling longer, they would have traveled much longer if they use the same routes as current HI users. Also, as shown in this example, the SO travelers would not divert too much, since SO travelers would choose the original routes (same as HI travelers) to maintain system performance if they need to divert too much. Additionally, we can see that as the penetration rate of PSOI increases, the average travel time of HI travelers also decreases. This further confirms the effectiveness of PSOI in traffic management.

Moreover, the travel time standard deviations of SO travelers are generally smaller than HI travelers, and it indicates that the SO information can give travelers a more consistent travel experience. Also, from the perspective of all travelers, more SO penetration can generally reduce the travel time standard deviation and thus makes the travel of all travelers in the network more consistent. This is an added benefit of PSOI provision for traffic management, in addition to travel time reduction, in improving the equity in the transportation system.

Table 5-1 MOEs of PSOI Simulation Results under Different Demand Levels and Market Penetration Rates and Mixed Traffic

(a) PSOI performance without other information strategies

\begin{tabular}{|l|l|l|l|l|}
\hline Penetration Rate & Information Group & $\begin{array}{l}\text { Average Travel } \\
\text { time }\end{array}$ & Average Distance & $\begin{array}{l}\text { Travel Time } \\
\text { Standard Deviation }\end{array}$ \\
\hline
\end{tabular}




\begin{tabular}{|c|c|c|c|c|}
\hline \multicolumn{5}{|c|}{ Demand $=7200$} \\
\hline \multirow[t]{3}{*}{$25 \%$} & All & 21.2 & 10.4 & 8.9 \\
\hline & $\mathrm{HI}$ & 24.3 & 11.0 & 8.2 \\
\hline & $\mathrm{SO}$ & 12.2 & 8.8 & 1.7 \\
\hline \multirow[t]{3}{*}{$50 \%$} & All & 14.9 & 10.7 & 5.2 \\
\hline & $\mathrm{HI}$ & 17.0 & 11.0 & 6.3 \\
\hline & SO & 12.8 & 10.5 & 2.3 \\
\hline \multirow[t]{3}{*}{$75 \%$} & All & 13.1 & 11.5 & 2.8 \\
\hline & $\mathrm{HI}$ & 12.6 & 11.0 & 2.5 \\
\hline & SO & 13.3 & 11.7 & 2.9 \\
\hline \multirow[t]{3}{*}{$100 \%$} & All & 13.4 & 11.6 & 3.1 \\
\hline & $\mathrm{HI}$ & -- & - & -- \\
\hline & $\mathrm{SO}$ & 13.4 & 11.6 & 3.1 \\
\hline \multicolumn{5}{|c|}{ Demand $=10800$} \\
\hline \multirow[t]{3}{*}{$25 \%$} & All & 21.6 & 11.2 & 9.5 \\
\hline & $\mathrm{HI}$ & 23.1 & 10.0 & 10.3 \\
\hline & $\mathrm{SO}$ & 16.9 & 14.7 & 4.1 \\
\hline \multirow[t]{3}{*}{$50 \%$} & All & 17.4 & 12.2 & 6.4 \\
\hline & $\mathrm{HI}$ & 18.4 & 10.0 & 8.2 \\
\hline & $\mathrm{SO}$ & 16.4 & 14.4 & 3.4 \\
\hline \multirow[t]{3}{*}{$75 \%$} & All & 15.2 & 12.0 & 4.0 \\
\hline & HI & 15.7 & 10.0 & 5.5 \\
\hline & $\mathrm{SO}$ & 15.0 & 12.7 & 3.3 \\
\hline \multirow[t]{3}{*}{$100 \%$} & All & 14.8 & 12.0 & 3.1 \\
\hline & $\mathrm{HI}$ & -- & -- & -- \\
\hline & SO & 14.8 & 12.0 & 3.1 \\
\hline
\end{tabular}

(b) PSOI performance with other information strategies 


\begin{tabular}{|c|c|c|c|}
\hline Information Group & Average Travel time & Average Distance & $\begin{array}{l}\text { Travel Time Standard } \\
\text { Deviation }\end{array}$ \\
\hline \multicolumn{4}{|l|}{ PSOI $=0 \%$} \\
\hline HI (include VMS) & 24.4 & 11 & 10.6 \\
\hline PTI & 24.5 & 11.5 & 9.5 \\
\hline ERI & 24.5 & 11.5 & 9.7 \\
\hline PSOI & -- & -- & -- \\
\hline All & 24.5 & 11.2 & 10.2 \\
\hline \multicolumn{4}{|l|}{$\mathrm{PSOI}=20 \%$} \\
\hline HI (include VMS) & 17.1 & 11.7 & 10.1 \\
\hline PTI & 15.6 & 12.0 & 8.6 \\
\hline ERI & 19.0 & 12.6 & 11.8 \\
\hline PSOI & 13.6 & 11.0 & 5.1 \\
\hline All & 16.4 & 11.8 & 9.5 \\
\hline \multicolumn{4}{|l|}{$\mathrm{PSOI}=40 \%$} \\
\hline HI (include VMS) & 15.4 & 11.8 & 8.3 \\
\hline PTI & 18.0 & 12.8 & 9.5 \\
\hline ERI & 19.9 & 14.6 & 13.7 \\
\hline PSOI & 13.6 & 11.7 & 4.3 \\
\hline All & 16.1 & 12.5 & 9.1 \\
\hline \multicolumn{4}{|l|}{ PSOI $=60 \%$} \\
\hline $\mathrm{HI}$ & --- & -- & -- \\
\hline PTI & 15.7 & 12.2 & 5.2 \\
\hline ERI & 17.2 & 13.1 & 6.2 \\
\hline PSOI & 14.2 & 12.9 & 3.2 \\
\hline All & 15.1 & 12.8 & 4.5 \\
\hline
\end{tabular}


Next, we compare PSOI with other information strategies (HI, PTI, ERI and VMS) under different system parameters, such as demand levels (base case and 1.5 times), and penetration rate $(25 \%, 50 \%, 75 \%, 100 \%)$. The simulation results are shown in Figure 5-5. The "HI" line represents the base case condition without any information. "VMS" represents the deployment at the location shown in Figure 5-4 (a), assuming a 20\% diversion rate.

From the system performance perspective, the results show that under different demand levels and penetration rates, PSOI is able to achieve better total system performance and more consistency in travel time among all travelers. PTI and ERI have similar attributes in that they perform better with lower and medium penetration rates while becoming less efficient in improving system performance with high penetration rates. It is reasonable given the UE nature of these two types of information. Travelers select shortest routes for themselves and when a lot of travelers select shortest routes based on instantaneous travel time information at the same time, the less congested routes will become congested after a while. VMS could help reduce average travel time by a small margin, but it is greatly constrained by its limited number of locations and low diversion rates. On the contrary, the predictability and SO nature of PSOI can guarantee that travelers will divert only if the resulting system performance is better. In terms of the travel time standard deviation, high penetration of PTI and ERI may deteriorate the travel time consistency in the network probably due to the difference in travel time of early and late diverted travelers. Meanwhile, increasing PSOI penetration rate could improve travel time consistency. 


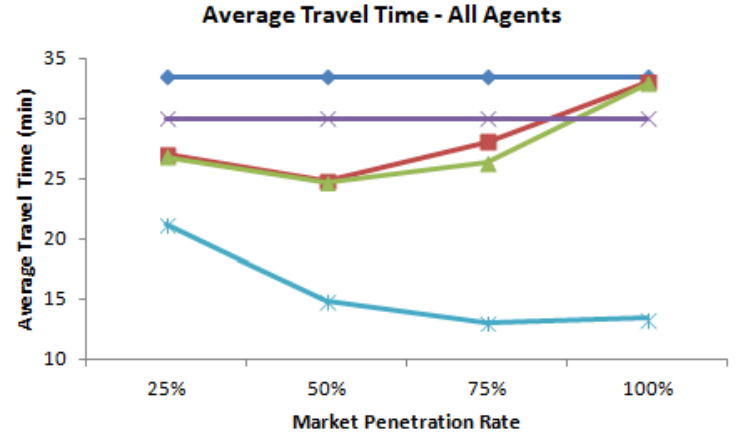

(a) Ave. travel time of all agents (demand =7200)

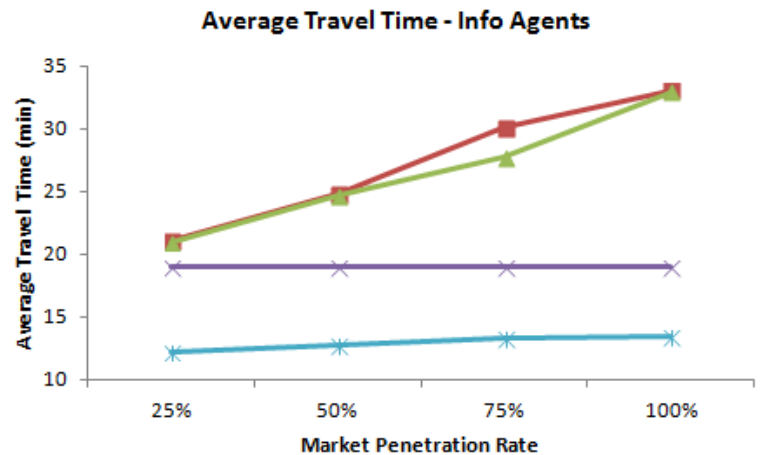

(c) Ave. travel time of info agents (demand $=7200$ )

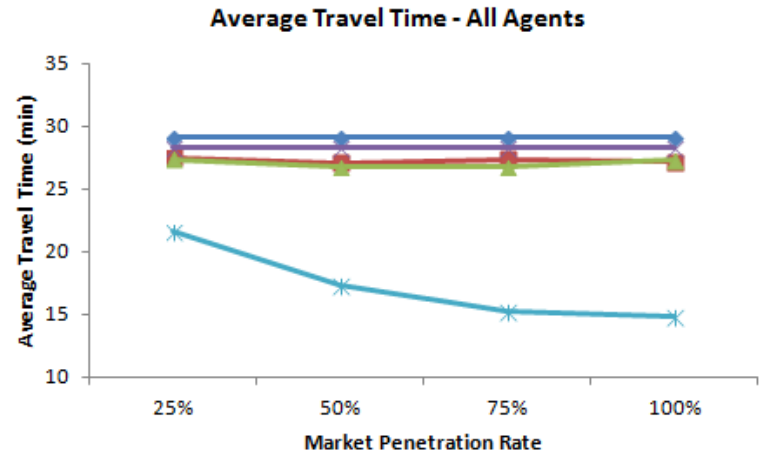

(e) Ave. travel time of all agents (demand $=10800$ )
Travel Time STD - All Agents

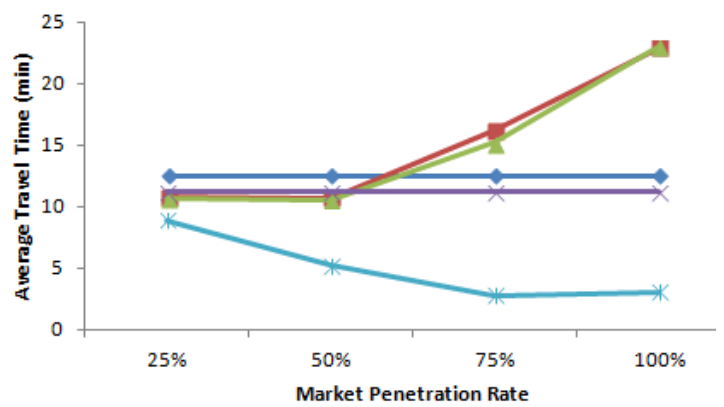

(b) Travel time STD of all agents (demand $=7200$ )

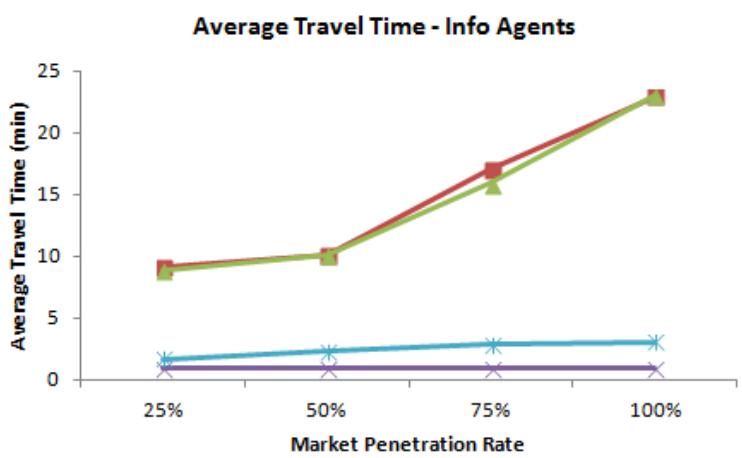

(d) Travel time STD of info agents (demand $=7200$ )

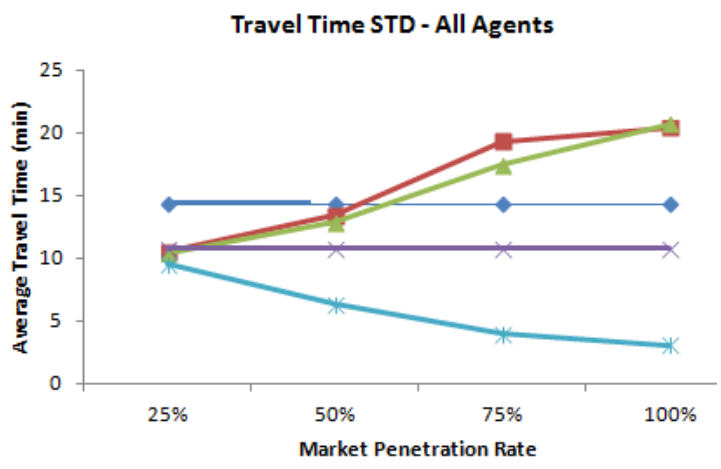

(f) Travel time STD of all agents (demand $=10800$ ) 


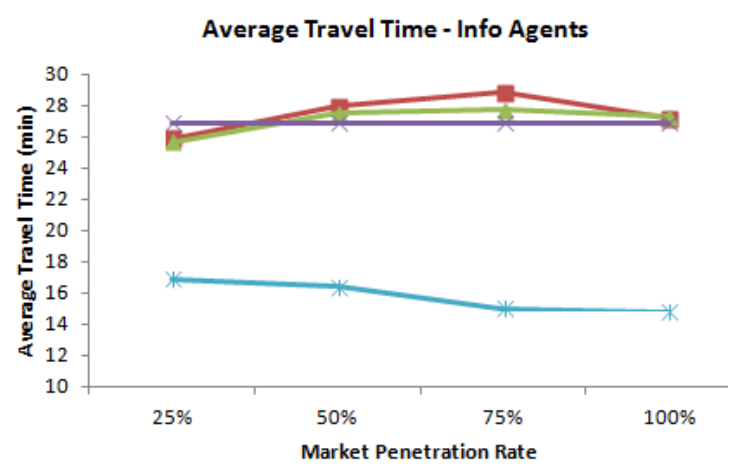

(g) Ave. travel time of info agents (demand $=10800$ )

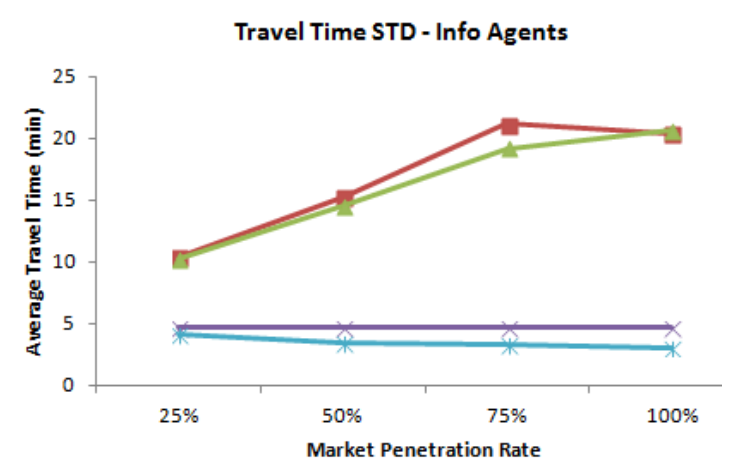

(h) Travel time STD of info agents (demand $=10800$ )

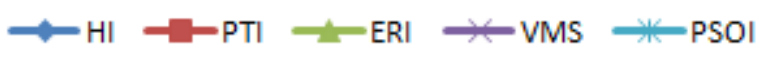

Figure 5-5 Sensitivity Analysis of Demand Levels and Market Penetration Rates.

From the perspective of informed travelers, PSOI also shows great benefits even compared with two UO information types (PTI and ERI). As penetration increases, the travel time of PTI and ERI travelers increase significantly due to simultaneous diversion to uncongested routes, while travel times of PSOI travelers are maintained at nearly the same travel time. The advantage of PSOI for its users makes it an attractive information mode for personal use, even though it is SO based information.

\section{Mixed Traffic}

It is also very interesting to investigate different information group users' experiences in mixed traffic. We set that $20 \%$ of all travelers receive PTI, 20\% ERI and 20\% PSOI. Also, for HI travelers, $20 \%$ of them will divert when they pass VMS located upstream of the incident as in Figure 5-4 (a). A typical algorithm convergence performance is shown in Figure 5-5 Sensitivity Analysis of Demand Levels and Market Penetration Rates.. For this simple network, the system state becomes relatively stable after 10th iteration. The fluctuation is due to changing behavior of PTI and ERI corresponding to new PSOI travelers' routes. Thanks to the small step size of the 
algorithm and simplicity of the network route structure, the fluctuation is very limited. This paper runs each algorithm for 30 iterations, records the solution of each iteration, and takes the results with lowest system average travel time as the final solution. The simulation results are shown in Error! Reference source not found. (b).

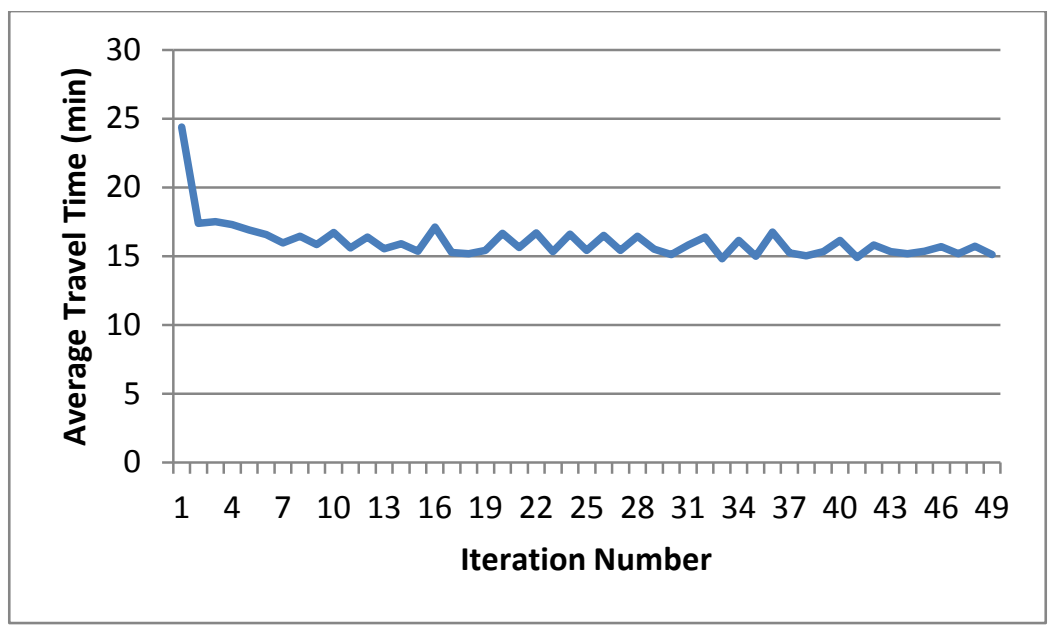

Figure 5-6 Algorithm Performance of Mixed Traffic Optimization for Three Corridor Network

As we can see, UO-based information groups, PTI and ERI, in this mixed traffic, are experiencing larger travel time value. In particular, as PSOI penetration increases, the travel time of PTI and ERI travelers become above the system average. This further reflects the drawback of using instantaneous travel time for route guidance, especially in a mixed information market, and raises questions about how much simple UE based information can help users. On the contrary, PSOI travelers always experience very short travel times, below system average. Further, as PSOI penetration increases, not only does the system average travel time for all travelers decrease, but the average travel time of HI, PTI and ERI increases as well. This is a particularly important attribute of PSOI and it further confirms its effectiveness in traffic management even when other types of information coexist. 


\section{Real World Network}

The next numerical experiment is performed on a real-world subarea network of Salt Lake City, Utah, which includes 149 nodes, 387 links, and 41 OD zones shown in Figure 5-7 (a). There are a total of 12638 trips/agents for a 2-hour typical evening peak hour period (3PM-5PM). The experiments were performed on the same computer as the first case studies. Each iteration of simulation run takes about 4.3 seconds of CPU time, which is fast for the network of this size.

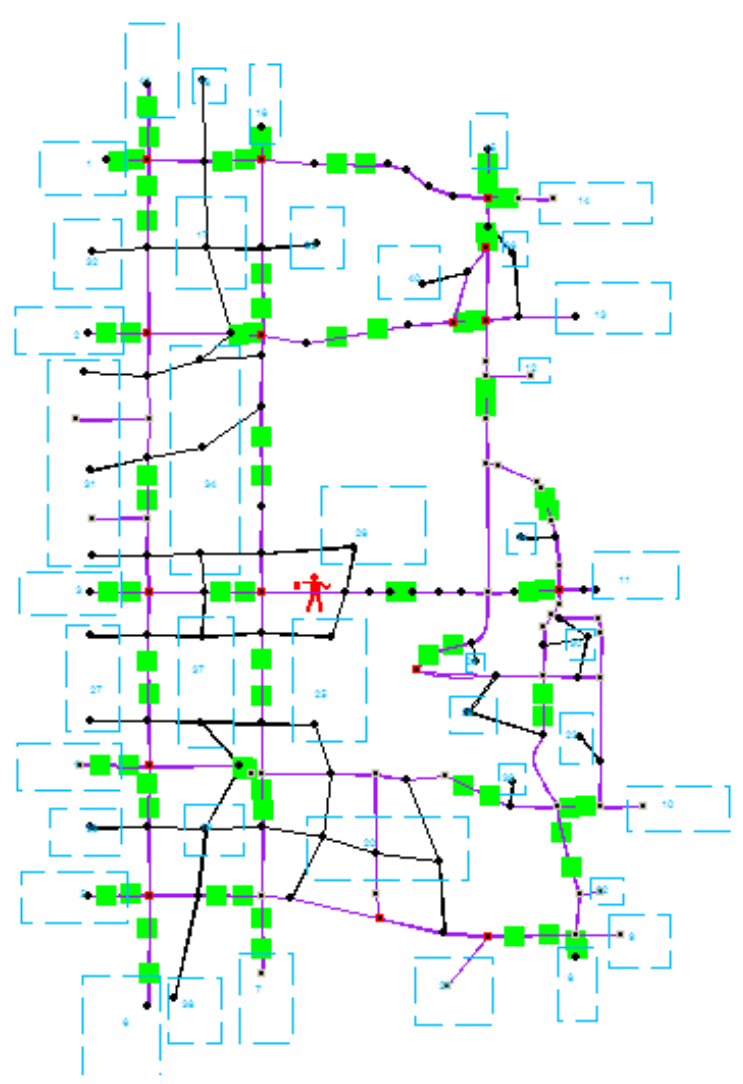

(a) Salt Lake City network, rectangles represent sensor locations for demand calibration 


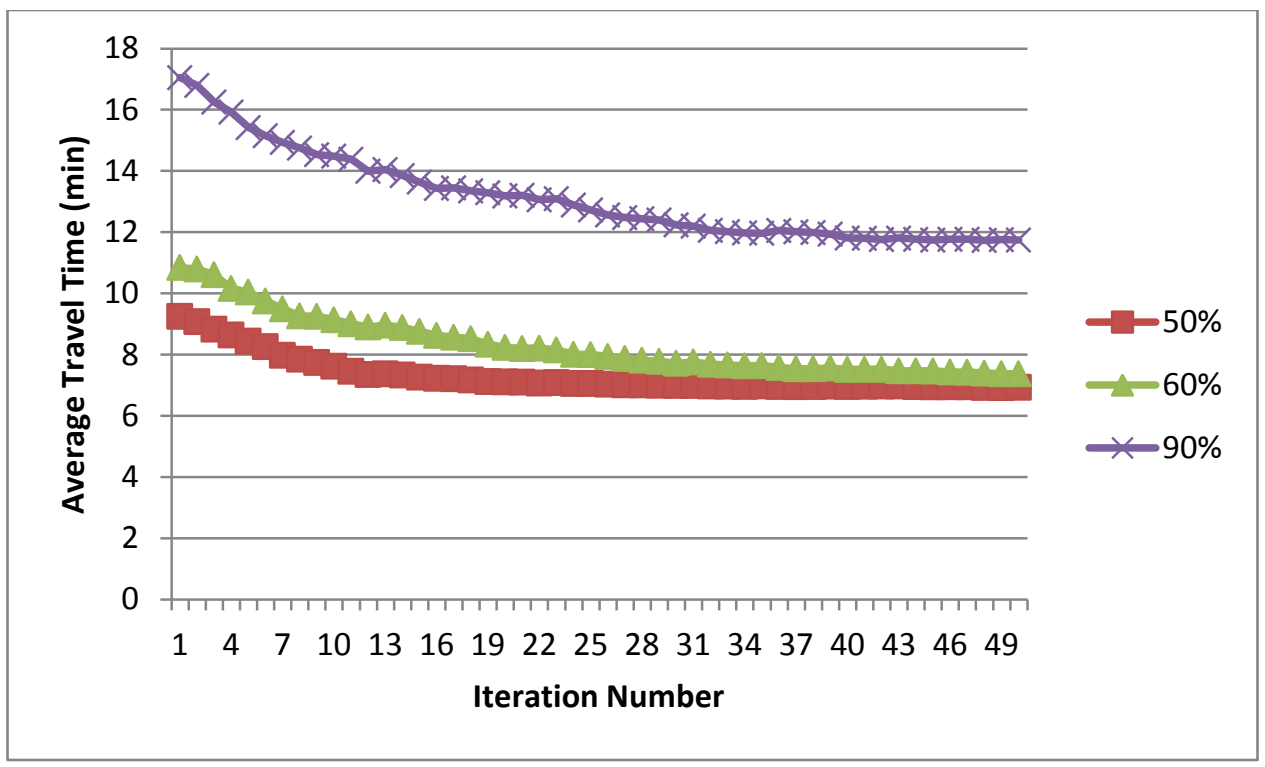

(b) Algorithm performance with different capacity reduction rate

Figure 5-7 Salt Lake City Network and Algorithm Performance

We simulate traffic conditions with a severe incident, such as accident causing road closure or hazmat spills on Highway 60, as in Figure 5-7 (a). The work zone caused capacity decrease for that link and lasted for 50 minutes. Figure 5-7 (b) shows the algorithm performance of three different roadway capacity reduction caused by incident: $50 \%, 60 \%$, and $90 \%$, using $25 \%$ as the PSOI market penetration (note all others are HI travelers). As we could see from Figure 5-7 (b), the algorithm is effective in reducing system average travel time. If $0.5 \%$ is set as algorithm stopping criteria, the algorithm would stop at the iteration of 13,18 , and 17 , indicating the algorithm's efficiency.

Sensitivity analyses are further conducted to examine the impact of PSOI market penetration for SLC subarea network, as shown in Figure 5-7 Salt Lake City Network and Algorithm Performance. The similar results are obtained as the three-corridor network. As PSOI penetration increases, the travel time and travel time standard deviation (STD) of all travelers 
decrease, and PSOI traveler's travel time is usually better than HI travelers. After a large penetration rate (e.g., 75\%), the benefit brought by PSOI information is only marginal.
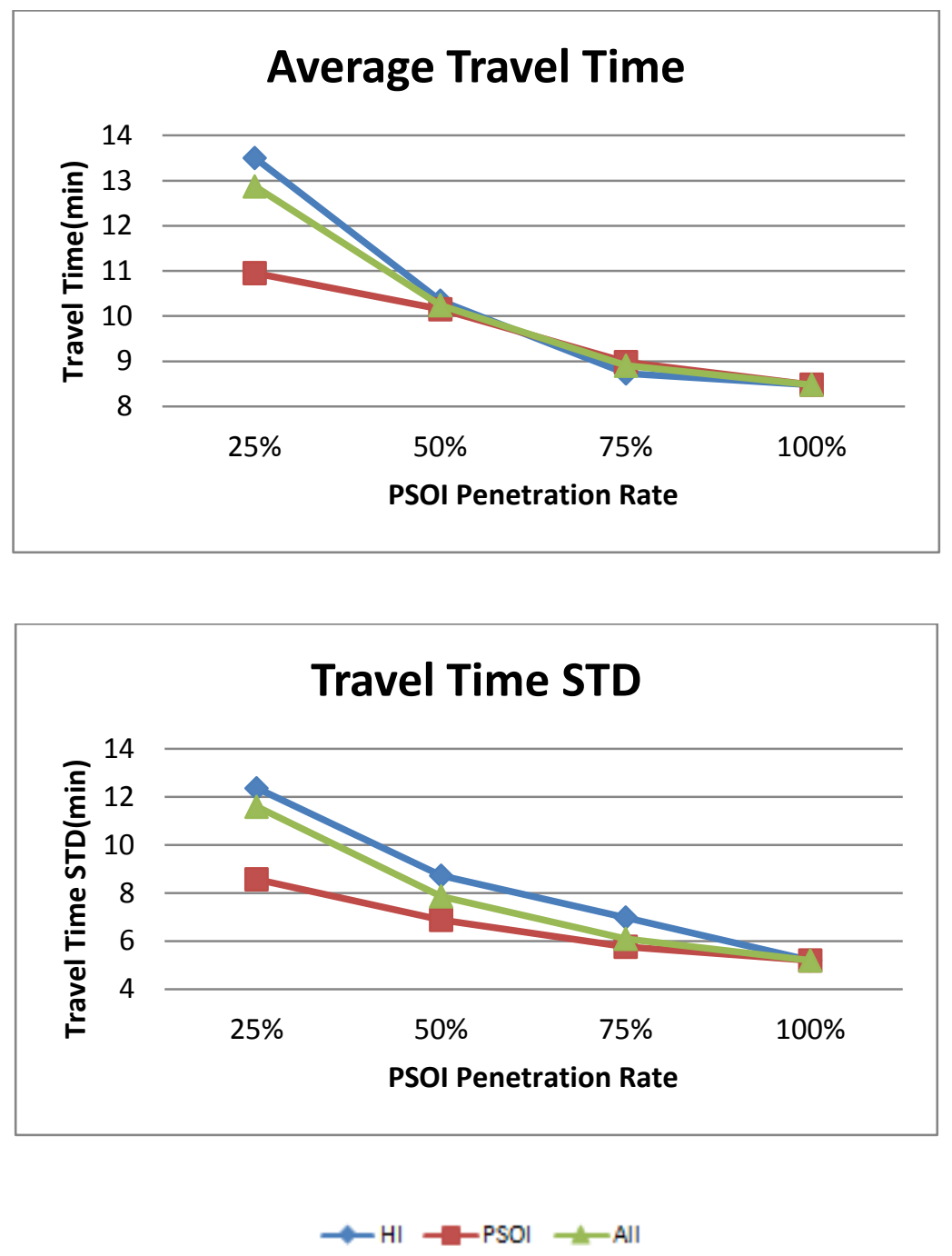

Figure 5-8 Sensitivity Analysis of PSOI Market Penetration Rate for SLC Subarea Network

For evaluation of mixed traffic condition, the same set-up are used as in the threecorridor network: $20 \%$ for PTI and ERI respectively. The algorithm performance is shown in Figure 5-9. There are obvious fluctuations in system performance from iteration to iteration. Compared with the relatively stable performance in Figure 5 for three-corridor network, this fluctuation is probably due to the complexity of network, resulting in many different route choice patterns. However, the simple queue-based algorithm successfully improved the system 
performance by $40 \%$ after 120 iterations (with computation time less than 5 min for this network, on the same computer as before).

Figure 9 demonstrates the sensitivity analyses of PSOI in mixed traffic for SLC subarea network. Similar conclusions are obtained to the three-corridor network. The increase in PSOI market penetration can reduce the travel time of all travelers in the network. More interestingly, PSOI users experience travel time as relatively short compared with other information users, making this information strategy acceptable as a personal information tool as well.

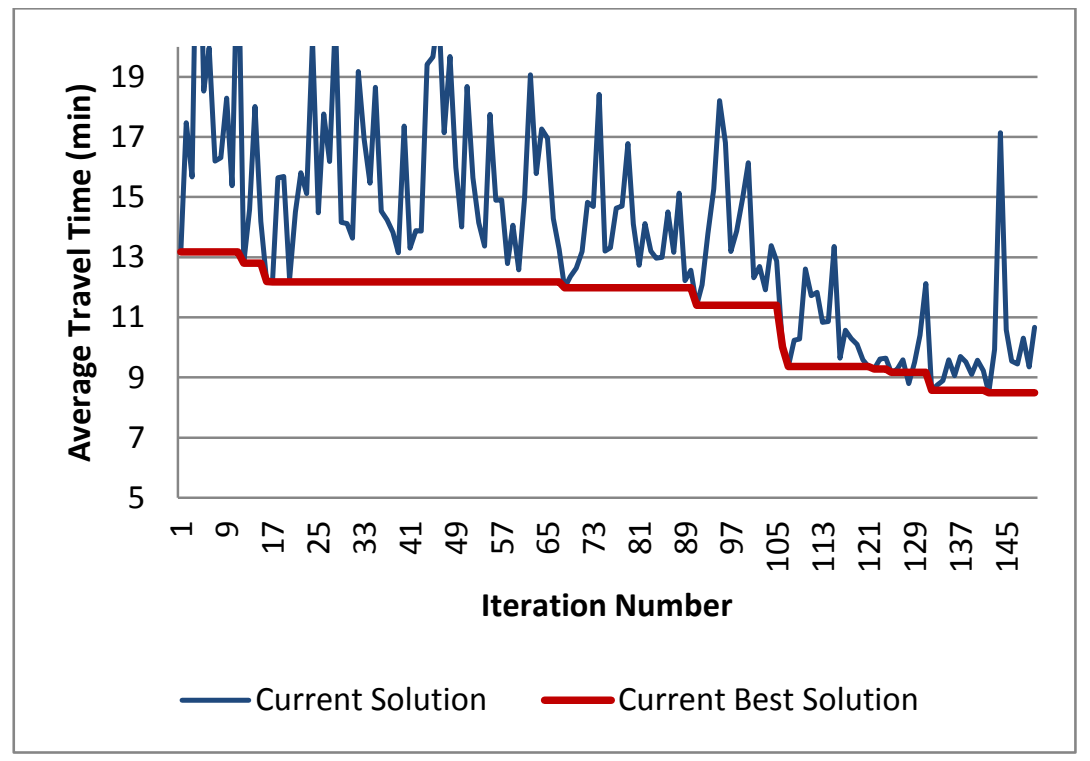

Figure 5-9 Algorithm Performance of Mixed Traffic Optimization for SLC Subarea Network 

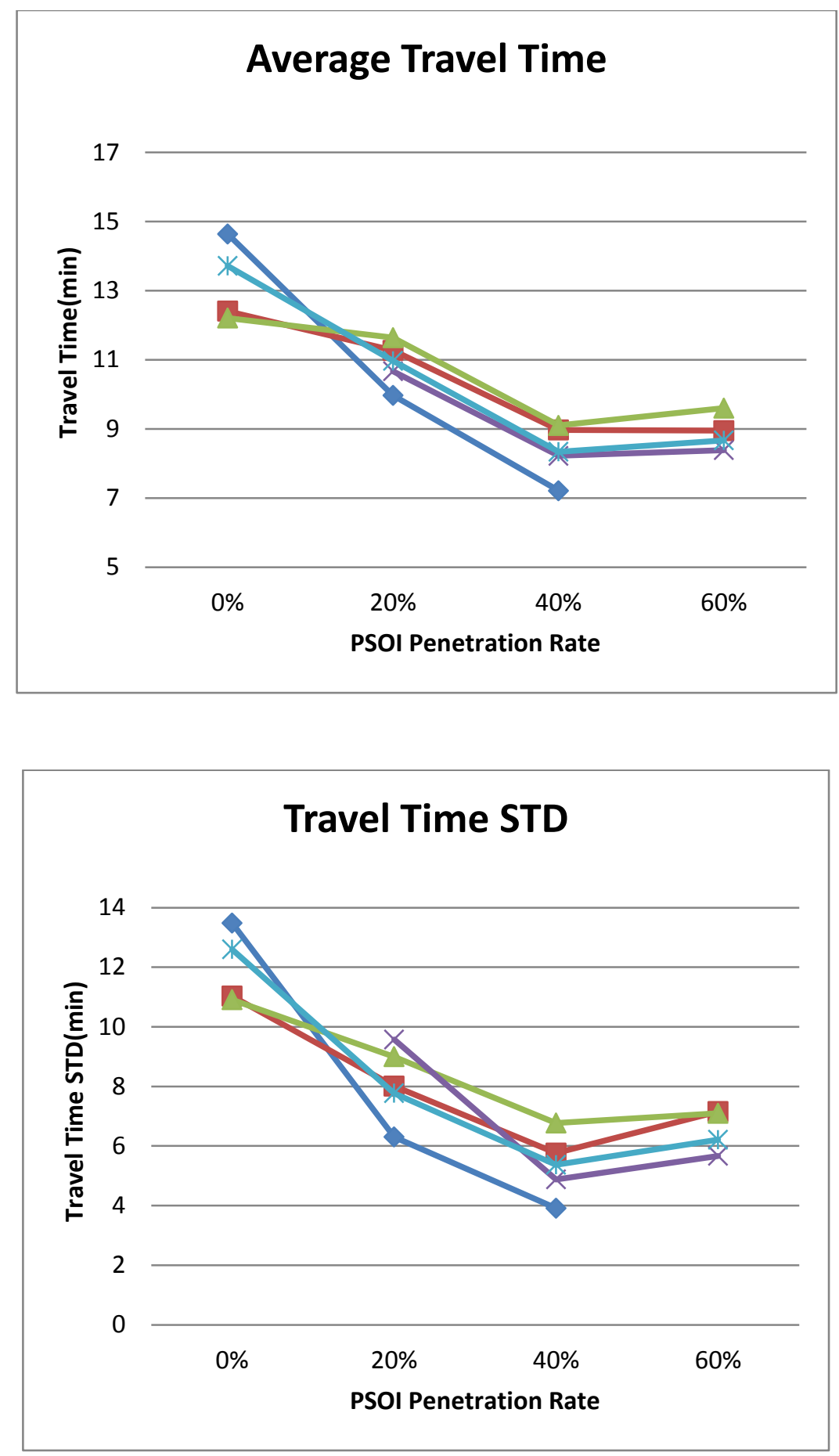

Figure 5-10 Sensitivity Analysis of PSOI in Mixed Traffic for SLC Subarea Network 


\subsection{Summary}

Among potential applications of VDMS for advanced traffic management, this chapter selects emergency routing as an example to showcase benefits from advanced traffic management enabled by VDMS. This chapter proposes a PSOI emergency routing strategy and developed efficient algorithm to generating optimal routing advisories to each individual traveler.

Case studies are carried out on a test network and a large real-world network. The proposed heuristic algorithm is proven effective in searching for near optimal PSOI solutions. Also, sensitivity analyses show that PSOI is not only an effective traffic management method in reducing average system travel time, but also an effective personal tool in travel guidance compared with UE-based PTI and ERI. Also, larger PSOI penetration can achieve shorter and more consistent average travel times for all travelers. Further, simulation results show that even in mixed traffic, PSOI is able to shorten travel time for both HI users and users of other information types. Thus, PSOI is recommended by this paper as an advantageous way for next generation advanced information systems and also dynamic traffic management. 


\section{CHAPTER 6 CONTRIBUTIONS AND FUTURE RESEARCH}

This research investigates a new concept VDMS to support next generation public traveler information. The concept leverages communication between vehicles and infrastructure. The development of first smartphone based Connected Vehicle traveler information application VDMS is conducted in this study. This research is also the first study the human factor issues regarding the effectiveness of VDMS concepts in delivering public messages. Additionally, a traffic management PSOI strategy is proposed and evaluated for emergency routing to demonstrate benefits of advanced traffic management strategies enabled by VDMS. Several areas for future research are identified that represent logical extensions of the ideas presented in this dissertation.

\subsection{Research Contributions}

This dissertation provided several contributions to the state-of-knowledge in advanced traveler information. Specific contributions include the following:

Innovative VDMS Concepts 
This research is the first work in the literature proposing and comprehensively evaluating the concept of VDMS. The concept is different from most of the current public and private information systems by possessing the following attributes.

- Scalable deployment: By using cyber infrastructure and personal devices, the information can be deployed at anywhere if needed, with only marginal added cost.

- Personalization: The information could be made targeting each individual traveler, based on their individual needs and attributes, such as current location, habitual routes, and value-of-time.

- Auditory messages: This allows more flexible in delivering messages, based on the desired message type, information units, contents, language and other personal set-ups.

- Better comprehension: as proven by this study, driver comprehension is usually better than DMSs with different messages and driving conditions

- Low distraction: as proven by this study, driver distraction of VDMS is usually lower than DMSs with different messages and driving conditions

- Dynamic traffic management strategies: with the flexibility of information delivery, more sophisticated information strategies could be applied to better dynamically manage real time traffic, particularly under special conditions.

\section{Prototype and Evaluation}

This research represents the first effort of development of connected vehicle traveler information app to demonstrate the proposed VDMS concept. This work is of particular importance since only subjectively accepted transportation telematics by the travelers can potentially become functional as designed.

The focused group user survey reveals a positive attitude among subjects toward VDMS in terms of both usefulness and satisfaction, with an average rating of -0.90 and -0.81 on a -2 to 2 
(Totally agree to Total disagree) five point Likert scale. The survey also indicates that potentially most drivers $(80.95 \%)$ perceive that VDMS is a safer way to receive information and most drivers $(66.67 \%)$ feel more comfortable receiving information from VDMS compared with DMS. The results indicate great user acceptability and potentials for such systems to be deployed by public agencies in the future.

\section{Effectiveness of Information Delivery by VDMS}

This research is the first effort to investigate the whether VDMS delivers information at least as well as current DMSs, so that we can know whether it is feasible for DOTs to transfer real-time traffic information dissemination to in-vehicle devices in the future from the perspective of message delivery, given its advantages in costs and scalability. Note that, this study is different from many previous studies investigating human factor issues for the message and display design of in-vehicle route guidance systems. This study makes unique contributions due to the unique attributes of VDMS concepts. The messages we are considering in this study are public traffic information similar to current DMS messages, instead of visual/auditory turn-by-turn information along with digital maps in a route-guidance system. The comprehension and distraction of information like turning instructions are potentially different from that of a common public message, such as location of incident and possible delays.

Analysis results revealed that VDMS generally performs better than DMS across different amounts of information and under different driving conditions, regardless of driver age.

- Message Comprehension: There are no significant differences for short messages and simple driving conditions. However, VDMS significantly better when information loads are high or when driving conditions became more complex. Also, VDMS can be used to provide longer messages than DMS under simple driving conditions since comprehension is not negatively impacted by message length for VDMS. 
- Driver distraction: Time to brake with VDMS was on average 0.39 seconds shorter than DMS under both simple and complex driving conditions. Complex driving conditions significantly increased time to brake for DMS, while time to brake did not significantly increase with driving complexity for VDMS.

- Subjective difficulty level: VDMS had a significantly lower perceived difficulty level for drivers than DMS except under simplest conditions (low information load and simple driving condition)

Based on these results, it is recommended that transportation agencies give careful consideration to VDMS as a future strategy for delivering public traffic information in a connected vehicle environment.

\section{Routing Advisories for Emergency Traffic Management}

With VDMS as a new way for public traveler information provision, many advanced informationbased strategies, such as routing advisory or speed advisory, are enabled to better manage traffic particularly under special conditions. The study takes the example of the routing advisory for emergency traffic management, develops and evaluates corresponding PSOI strategy, to demonstrate potential benefits.

An efficient queue-based heuristic algorithm is developed for generating VDMS routing advisories. The algorithm itself makes a few contributions to the literature: individual-based, efficient computational performance, and of predicative nature. Also, this study considers mixed information use in the traffic network and generated routing advisories are able to accommodate the adaptive behavior of other users in the network.

Case studies indicated that heuristic algorithm was effective, and existence of PSOI can help shorten average travel times for all types of travelers, with maximum benefits obtained after a certain market penetration ( $75 \%$ in our case studies). Also, sensitivity analyses show that PSOI 
is not only an effective traffic management method in reducing average system travel time, but also an effective personal tool in travel guidance compared with other information groups, even with UO-based PTI and ERI. Further, simulation results show that even in mixed traffic, PSOI is able to shorten travel time for both HI users and users of other information groups. Thus, PSOI is recommended by this paper as an advantageous way for next generation advanced information systems enabled by VDMS, particularly for emergency traffic management by Traffic Management Centers.

\subsection{Future Research}

In the course of this research, several topics were identified as areas of future research. They are listed here, sorted by general topic area.

\section{Human Factors}

Since this was the first study to evaluate the feasibility and effectiveness of VDMS, there are still some limitations and unanswered questions to be explored in the future. First, although driving simulators are commonly used in traffic research due to their practicality and high level of experimental control, replications of the study in real-life driving settings, such as via on-road assessments, are needed in order to ensure the generalizability of the findings. Second, the current experimental design is simplified in some aspects (e.g., two levels for each factor), and ignores some other factors (e.g., gender, education level). Further studies are needed to investigate more factors with more levels, such as higher levels of information. Third, the VDMS auditory message in this paper are only audio version of similar visual DMS messages, while in the practice there are other strategies to make VDMS messages more effective, such as repeating key and difficult information twice, which is not achievable in DMS. The effectiveness of these improvements should be further evaluated as well to improve the VDMS design. Also, displaying 
more complex messages (e.g., maps/visual texts on an in-vehicle device screen along with detailed auditory messages) are not considered in this paper. Further studies can be conducted whether it is appropriate for DOTs to provide detailed information while limiting the distractions to reasonable level.

Next, there are other human factor issues for VDMS messages, such as characteristics of the voice and structure of the auditory messages, are issues that need to be addressed in future research. Another possible future item would be to examine levels of ambient noise and their effect on driver performance with VDMS. Last, this study used scenarios on a local network that subjects would be familiar with. It is important to investigate whether results hold for non-local drivers traveling an unfamiliar network.

\section{Advanced Applications of VDMS}

Future research can address other potential applications of VDMS in traffic management, such as speed advisories for speed harmonization, lane change advisories for merge control, mode suggestions, and routing advisories for green transportation. Although similar topics have been explored in the literature multiple times, the unique attributes of VDMS brings more capabilities for these strategies, which can be potentially more effective in achieving respective goals, by understanding the attributes of each individual in terms of location, destination, and value-of-time, etc. A recent funding opportunities of US Department of Energy (www.arpa-e.energy.gov) challenges applicants to develop mechanisms for individual travelers that both signal and guide them toward improvement of the energy efficiency of the transportation network in multimodal urban areas. This is a good example of potential VDMS applications.

Future research can also further extend and improve the routing advisory algorithm proposed this study. The proposed algorithm can be further extended and incorporated in a multi- 
period rolling horizon approach. We can also consider robust information provision by considering variables such as random incident duration and compliance rate, by using techniques such as stochastic programming. 


\section{REFERENCES}

Abbas, M. M., and P. T. McCoy. Optimizing Variable Message Sign Locations on Freeways Using Genetic Algorithms. Presented at the 78th Annual Meeting of the Transportation Research Board, Washington, D.C., 1999.

Adler, J. L., \& Blue, V. J. (2002). A cooperative multi-agent transportation management and route guidance system. Transportation Research Part C: Emerging Technologies, 10(5), 433 454.

Atchley, P., \& Chan, M. (2011). Potential benefits and costs of concurrent task engagement to maintain vigilance: A driving simulator investigation. Human Factors, 53(1), 3-12.

Ayça Berfu Ünal, Dick de Waard, Kai Epstude, Linda Steg. (2013) Driving with music: Effects on arousal and performance. Transportation Research Part F: Traffic Psychology and Behaviour, Volume 21, Pages 52-65

Becker, S., Brockmann, M., Mertens, A., Nui, R. and Sonntag, A. (1995) User acceptance and willingness to pay for advanced driver support systems. Paper presented at TRAFFIC Technology Europe '95, Berlin.

Ben-Akiva, M. E., Gao, S., Wei, Z., \& Wen, Y. (2012). A dynamic traffic assignment model for highly congested urban networks. Transportation Research Part C: Emerging Technologies, 24, 62-82.

Bierlaire, M., Chen, J., \& Newman, J. (2013). A probabilistic map matching method for smartphone GPS data. Transportation Research Part C: Emerging Technologies, 26, 78-98.

Bolellia, V. Mauro, and E. Perono, (1991) "Models and Strategies for Dynamic Route GuidancPart B: A Decentralized, Fully Dynamic, Infrastructure Supported Route Guidance,” in Proc. DRIVE Conference, Brussels, Belgium, 99-105.

Bousquet, P, Peck, S. (2008). A technology comparison of two in-vehicle warning methods at level crossings with human factor implications World Level Crossing Symposium, 10th, 2008, Paris, France.

Boyd, S., \& Vandenberghe, L. (2009). Convex optimization. Cambridge university press.

Brazil, W., B. Caulfield, and N. Rieser-Schussler. (2013) Understanding carbon: Making emissions information relevant. Transportation Research Part D: Transport and Environment, Volume 19, Pages 28-33

Brookhuis, K. A., De Vries, G., \& De Waard, D. (1991). The effects of mobile phone telephoning on driving performance. Accident Analysis and Prevention, 23(4), 309-316. 
Burnett, G., and S. Joyner. (1997). An Assessment of Moving Map and Symbol-Based Route Guidance Systems. In Ergonomics and Safety of Intelligent Driver Interfaces (Y. I. Noy, ed.), Lawrence Erlbaum Associates, Mahwah, N.J., pp. 115-138.

Campbell, J. L., C. Carney, and B. H. Kantowitz. (1997). Development of Human Factors Guidelines for Advanced Traveler Information Systems (ATIS) and Commercial Vehicle Operations (CVO). Draft report. FHWA, Washington, D.C.

Carifio, J. \& Perla, R. (2007). Ten Common Misunderstandings, Misconceptions, Persistent Myths and Urban Legends about Likert Scales and Likert Response Formats and their Antidotes. Journal of Social Sciences, 2, 106-116.

Charbonnier, C., Farges, J.L. and J.J. Henry. 1991. Models and Strategies for Dynamic Route Guidance-Part C: Optimal Control Approach. Proceedings of the DRIVE Conference, Brussels, Belgium, pp. 106-112.

Chen, X. M., Zhang, L., He, X., Xiong, C., \& Li, Z. (2014). Surrogate Based Optimization of Expensive-to-Evaluate Objective for Optimal Highway Toll Charges in Transportation Network. Computer - Aided Civil and Infrastructure Engineering, 29(5), 359-381.

Chiu, Y. C., Zheng, H., Villalobos, J. A., Peacock, W., \& Henk, R. (2008). Evaluating regional contra-flow and phased evacuation strategies for Texas using a large-scale dynamic traffic simulation and assignment approach. Journal of Homeland Security and Emergency Management, 5(1).

Chiu, Y-C., N. Huynh, and H. S. Mahmassani. Location configuration design for Dynamic Message Signs under stochastic incident and ATIS scenarios. Transportation Research Part C 15, pp. 33-50, 2007.

Cohen, J. (1969). Statistical power analysis for the behavioural sciences. New York: Academic Press.

Cohen, J. (1973). Eta-squared and partial eta-squared in fixed factor ANOVA designs. Educational and psychological measurement, 33, 107-112.

Cohen, J. (1988). Statistical power analysis for the behavioral sciences. Hillsdale, New Jersey: Lawrence Erlbaum Associates.

Crosby, P., Spyridakis, J., Ramey, J., Haselkorn, M. and Barfield, W. (1993) A primer on usability testing for developers of travel information systems. Transportation Research C 2 , 143-I 57.

Daganzo, C. F. 1994. "The Cell Transmission Model: A Dynamic Representation of Highway Traffic Consistent with the Hydrodynamic Theory." Transportation Research B 28 (4): 269287.

Daganzo, C. F. 1995. “The Cell Transmission Model, Part II: Network Traffic.” Transportation Research B 29 (2): 79-93. 
Dalton P., P. Agarwal, N. Fraenkel, J. Baichoo, A. Masry. (2013) Driving with navigational instructions: Investigating user behaviour and performance Accident Analysis \& Prevention, Volume 50, Pages 298-303

Davidsson, P., Persson, J. A., \& Holmgren, J. (2007). On the integration of agent-based and mathematical optimization techniques. In Agent and multi-agent systems: technologies and applications (pp. 1-10). Springer Berlin Heidelberg.

De Waard, D., \& Brookhuis, K. A. (1997). On the measurement of driver mental workload. In J. A. Rothengatter \& E. Carbonell Vaya (Eds.), Traffic and transport psychology (pp. 161-171). Amsterdam, The Netherlands: Pergamon.

Dia, H. (2002). An agent-based approach to modelling driver route choice behaviour under the influence of real-time information. Transportation Research Part C: Emerging Technologies, 10(5), 331-349.

Dudak, C. L. (1970). State of the Art Related to Real Time Traffic Information for Urban Freeways. Texas Transportation Institute, College Station, Texas.

Dudek, C. L., and G. L. Ullman. (2002). Flashing Messages, Flashing Lines, and Alternating One Line on Changeable Message Signs. In Transportation Research Record: Journal of the Transportation Research Board, No. 1803, Transportation Research Board of the National Academies, Washington, D.C., pp. 94-101.

Dudek, C. L., and R. D. Huchingson. (1986). Manual on Real-Time Motorist Information Displays. FHWA-IP-86-16. FHWA, U.S. Department of Transportation, Washington, D.C.

Dudek, C. L., and Ullman, G. L. (2006). Dynamic message sign message design and display manual, Texas Transportation Institute, College Station, TX.

Dudek, C. L., N. Trout, B. Durkop, S. Booth, and G. L. Ullman. (2000). Improving Dynamic Message Sign Operations. FHWA/TX-01/1882-2. Texas Transportation Institute, College Station, TX.

Dudek, C. L., S. D. Schrock, and G. L. Ullman. (2005). Impacts of Using Dynamic Features to Display Messages on Changeable Message Signs. FHWAHOP-05-069. FHWA, U.S. Department of Transportation, Washington, D.C.

Edelman, B., Valentin, D., \& Dowling, W. J. (2009). Experimental design and analysis for psychology. Oxford: Oxford University Press.

Fastenmeier, W., R. Haller, and G. Lerner. (1994). A Preliminary Safety Evaluation of Route Guidance Comparing Different MMI Concepts. Proc., First ATT/IVHS World Congress, Vol. 4, pp. 1750-1757.

Faul, F., Erdfelder, E., Lang, A.-G., \& Buchner, A. (2007). G*Power 3: A flexible statistical power analysis for the social, behavioral, and biomedical sciences. Behavior Research 
Methods, 39, 175-191.http://www.psycho.uni-

duesseldorf.de/abteilungen/aap/gpower3/download-and-register

Gaffuri, J. (2006). How to merge optimization and agent-based techniques in a single generalization model. In workshop on generalisation and multiple representation, Vancouver, United-States.

Gao, S., \& Huang, H. (2012). Real-time traveler information for optimal adaptive routing in stochastic time-dependent networks. Transportation Research Part C: Emerging Technologies, 21(1), 196-213.

Ghali, M. O., \& Smith, M. J. (1995). A model for the dynamic system optimum traffic assignment problem. Transportation Research Part B: Methodological, 29(3), 155-170.

Gjerdrum, J., Shah, N., \& Papageorgiou, L. G. (2001). A combined optimization and agent-based approach to supply chain modelling and performance assessment. Production Planning \& Control, 12(1), 81-88.

Glass, Peckham, and Sanders (1972). Consequences of failure to meet assumptions underlying the analyses of variance and covariance, Review of Educational Research, 42, 237-288.

Goodall, N. J., B. L. Smith, and B. Park. (2013) Traffic Signal Control with Connected Vehicles. Journal of the Transportation Research Board, National Research Council, Washington, D.C.

Gretchen Paelke \& Paul Green. Entry of Destinations into Route Guidance Systems: A Human Factors Evaluation.

Hancock, P. A., \& Verwey, W. B. (1997). Fatigue, workload and adaptive driver systems. Accident Analysis and Prevention, 29(4), 495-506.

Hatfield J. \& Chamberlain T. (2008). The effect of audio materials from a rear-seat audiovisual entertainment system or from radio on simulated driving. Transportation Research Part F: Traffic Psychology and Behaviour, Volume 11, Issue 1, Pages 52-60.

Hawas Y, and H. S. Mahmassani, (1995) “A Decentralized Scheme for Real-Time Route Guidance in Vehicular Traffic Networks," in Proc. 2nd World Congress of Intelligent Transport Systems, Yokohama, Japan, 1956-1963.

Hawas Y., (2012) "A Cooperative Distributed System for Real-Time Route Guidance," Journal of Transportation Technologies, Vol. 2 No. 3, pp. 230-240.

Hobeika, A., Sivanandan, R., Jehanian, K. M., \& Ameen, M. D. (1996). Advanced traveler information system users' needs in I-95 Northeast Corridor. Transportation Research Record: Journal of the Transportation Research Board, 1537(1), 55-62.

Horrey, W. J., \&Wickens, C. D. (2004). The impact of cell phone conversations on driving: A metaanalytic approach. Report No. AHFD-04-2/GM-04-1. Aviation Human Factors Division, University of Illinois, Savoy, USA. 
Howell, W. C. (2006). Simulation optimization of traffic light signal timings via perturbation analysis.

Huynh, N., Chiu, Y.-C., and Mahmassani, H.S., "Finding Near-Optimal Locations for Variable Message Signs for Real-Time Network Traffic Management”, Transportation Research Record1856, pp.34-53, 2003.

Jahn, O., Möhring, R. H., Schulz, A. S., \& Stier-Moses, N. E. (2005). System-optimal routing of traffic flows with user constraints in networks with congestion. Operations Research, 53(4), 600-616.

Jayakrishnan, R., Mahmassani, H. S., \& Hu, T. Y. (1994). An evaluation tool for advanced traffic information and management systems in urban networks. Transportation Research Part C: Emerging Technologies, 2(3), 129-147.

Jayakrishnan, R., Mahmassani, H. S., \& Hu, T. Y. (1994). An evaluation tool for advanced traffic information and management systems in urban networks. Transportation Research Part C: Emerging Technologies, 2(3), 129-147.

Jia A. Zhou, X. ,Li. M. Rouphail, N., Williams, B. (2011) Incorporating Stochastic Road Capacity into a Day-to-Day Traffic Simulation and Traveler Learning Framework: Model Development and Case Study. Journal of Transportation Research Board. 11-2820, pp 112121.

Jia, A., Zhou, X., \& Rouphail, N. M. (2013) "Agent-Based Approach to Pricing Strategy Evaluation: Incorporation of Drivers' Heterogeneity, Departure Time Shift and Comprehensive Learning Model". In Transportation Research Board 92nd Annual Meeting (No. 13-4126).

Jin, W., C. Kwan, Z. Sun, H. Yang, and Q. Gan. (2012) SPIVC: Smart-Phone-Based Inter-vehicle Communication System, Proceedings of Transportation Research Board Annual Meeting, Washington, D.C.

Jonathan F. Antin, Laura M. Stanley, \& Kathleen F. Cicora. (2010). Conventional Versus Moving-Map Navigation Methods. Transportation Research Record: Journal of the Transportation Research Board, Volume 2138, pp 34-41.

Kelly, Michael J, Stanley, Laura M, \& Lassacher, Suzanne. (2005). Driver Performance While Using A Cellular Telephone Interface To A Traveler Information System. Western Transportation Institute, MT, 33p.

Khattak, A. J., Pan, X., Williams, B., Rouphail, N., \& Fan, Y. (2008). Traveler information delivery mechanisms: Impact on consumer behavior. Transportation Research Record: Journal of the Transportation Research Board, 2069(1), 77-84.

Khattak, Asad J., Joseph L. Schofer and Mu-Ham Wang. (1995) “A Simple Time Sequential Procedure for Predicting Freeway Incident Duration.” IVHS Journal. Vol. 2, No.2, pp 113-138. 
Kuiken, M. J. and Groeger, J. A. (1993) Effects of feedback on driving performance at crossroads and on curves. Report VK 93-12, Haren, University of Groningen, Traffic Research Centre, The Netherlands.

Lee, J., \& Park, B. B. (2008). Evaluation of route guidance strategies based on vehicleinfrastructure integration under incident conditions. Transportation Research Record: Journal of the Transportation Research Board, 2086(1), 107-114.

Lee, J., B. Park, K. Malakorn, and J. So. (2013) Sustainability Assessments of Cooperative Vehicle Intersection Control at an Urban Corridor. Transportation Research, Part C, Vol. 32, pp. 193-206

Liao, C. (2013) Using Smartphone App to Support Visually Impaired Pedestrians at Signalized Crossings. In Transportation Research Record: Journal of the Transportation Research Board, No. 0231, TRB, National Research Council, Washington, D.C.

Lu, C. C., Mahmassani, H. S., \& Zhou, X. (2008). A bi-criterion dynamic user equilibrium traffic assignment model and solution algorithm for evaluating dynamic road pricing strategies. Transportation Research Part C: Emerging Technologies, 16(4), 371-389.

Lu, C. C., Mahmassani, H. S., \& Zhou, X. (2009). Equivalent gap function-based reformulation and solution algorithm for the dynamic user equilibrium problem. Transportation Research Part B: Methodological, 43(3), 345-364.

Lu, Chung-Cheng, Xuesong Zhou, Kuilin Zhang. (2013). Dynamic origin-destination demand flow estimation under congested traffic conditions. Transportation Research Part C: Emerging Technologies, Volume 34, Pages 16-37.

Mahmassani, H.S., Jayakrishnan, R.(1999) System performance and user response under realtime information in a congested traffic corridor. Transportation Research Part A 25 (5), 293 308.

Meng, L., \& Zhou, X. (2011). Robust single-track train dispatching model under a dynamic and stochastic environment: A scenario-based rolling horizon solution approach. Transportation Research Part B: Methodological, 45(7), 1080-1102.

Nam, D., Mannering, F., 2000. An exploratory hazard-based analysis of highway incident duration. Transportation Research Part A 34 (2), 85-102.

Newell, G. F., 1993. A simplified theory on kinematic waves in highway traffic, part I: general theory. Transportation Research Part B 27(4), pp. 281-287.

Osorio, C., \& Bierlaire, M. (2010). A simulation-based optimization framework for urban traffic control. Report TRANSP-OR, 101203.

Ozbay, K., Kachroo, P., 1999. Incident Management in Intelligent Transportation Systems. Artech House, Boston, MA. 
Park, H. and Smith, B. (2012). Investigating Benefits of IntelliDrive in Freeway Operations: Lane Changing Advisory Case Study. J. Transp. Eng., 138(9), 1113-1122.

Parkes, A. M., and G. E. Burnett. (1993). An Evaluation of Medium Range Advance Information in Route-Guidance Displays for Use in Vehicles. Proc., 4th International IEEE Vehicle Navigation and Information Systems Conference, pp. 238-241.

Paz, A., Peeta, S. (2007) Behavior-consistent real-time traffic routing under information provision. In: Proceedings of the 86th Annual Meeting of the Transportation Research Board, Washington, DC. [CD-ROM].

Paz, A., Peeta, S. (2007) Behavior-consistent real-time traffic routing under information provision. In: Proceedings of the 86th Annual Meeting of the Transportation Research Board, Washington, DC. [CD-ROM].

Peeta, S. and Gedela, S. (2001). "Real-Time Variable Message Signs Based Route Guidance Consistent With Driver Behavior,“ Transportation Research Record, 1752, pp. 117-125.

Peeta, S. and Gedela, S. (2001). "Real-Time Variable Message Signs Based Route Guidance Consistent With Driver Behavior,“ Transportation Research Record, 1752, pp. 117-125.

Peeta, S. and Mahmassani, H.S. (1995). "Multiple User Classes Real-Time Traffic Assignment for On-Line Operations: A Rolling Horizon Solution Framework," Transportation Research, 3C, No. 2, pp. 83-98.

Peeta, S., Ramos, J.L., and Pasupathy, R. (2000). "Content of Variable Message Signs and Online Driver Behavior,“ Transportation Research Record, 1725, pp. 102-108.

Persson, J. A., Davidsson, P., Johansson, S. J., \& Wernstedt, F. (2005). Combining Agent-Based Approaches and Classical Optimization Techniques. In EUMAS (pp. 260-269).

Roelofs, J. C. (1987). Synthetic Speech in Practice: Acceptance and Efficiency. Behavior and Information Technology, Vol. 6, No. 4, 1987, pp. 403-410.

Rothengatter, J. A., De Waard, D., Slotegraaf, G., Carbonell Vaya, E. and Muskaug, R. (1991) Social Acceptance of Automatic Policing and Information Systems. Report 1033/AUTOPOLIS/Deliverable 7. University of Groningen, Traffic Research Centre, The Netherlands.

Schafer, J. L., Khattak, A. and Koppelman, F. S. (1993) Behavioral issues in the design and evaluation of advanced traveler information systems. Transportation Research C 2, 107-I 17.

Schleiffer, R. (2002). Intelligent agents in traffic and transportation. Transportation Research Part C: Emerging Technologies, 10(5), 325-329.

Schraagen, J. M. C. Information Presentation in In-Car Navigation Systems. In Driving Future Vehicles (A. M. Parkes and S. Franzen, eds.), Taylor \& Francis, London, 1993, pp. 171-185 
Schroeder, J. and Demetsky, M. (2011). "Data Analysis of Diversion and Dynamic Message Signs." J. Transp. Eng., 137(9), 608-614.

Sheffi, Y. (1985). Urban transportation networks: equilibrium analysis with mathematical programming methods. Prentice-Hall, Englewood Cliffs, NJ.

Sheffi, Y. (1985). Urban transportation networks: equilibrium analysis with mathematical programming methods.

Shen, W., Nie, Y., \& Zhang, H. M. (2006). Path-based system optimal dynamic traffic assignment models: formulations and solution methods. In Intelligent Transportation Systems Conference, 2006. ITSC'06. IEEE (pp. 1298-1303).

Smith, K., Smith, B., 2001. Forecasting the Clearance Time of Freeway Accidents. Research Report. STL-2001-01. Center for Transportation Studies, University of Virginia, Charlottesville, VA.

Srinivasan, R., P. Jovanis, F. Landau, M. Laur, C. Lee, and C. M. Hein. (1995). California Advanced Driver Information System II (CADIS II): Extended Tests of Audio and Visual Route Guidance Displays. Report UCD-ITS-RR-95-3. Institute of Transportation Studies, University of California, Davis.

Srinivasan, Raghavan and Jovanis, Paul. (1998). Advanced Traveler Information Systems: Driver Safety, Perceptions and Preferences. Institute of Transportation Engineers. Meeting (68th : 1998 : Toronto Ont.). Annual meeting papers [CD-ROM], 18p.

Srinivasan, Raghavan. (1999). Overview of Some Human Factors Design Issues for In-Vehicle Navigation and Route Guidance Systems. Transportation Research Record: Journal of the Transportation Research Board, 1694, pp 20-29

Sullivan, E.C., 1997. New model for predicting incidents and incident delay. ASCE Journal of Transportation Engineering 123 (4), 267-275.

Swaya O. B., Doan D. L., Ziliaskopoulos A. K., and Fourer R. (2001) Multistage Stochastic System Optimum Dynamic Traffic Assignment Program with Recourse for Incident Traffic Management. Transportation Research Record: Journal of the Transportation Research Board, volume 1748, pp 116-124.

Teigen, K. H. (1994). Yerkes-Dodson: A law for all seasons. Theory Psychology, 4(4), 525-547.

Ullman, G. L., and C. L. Dudek. (1999) Legibility Distance of Light-Emitting Diode (LED) Variable Message Signs. Proc., Sixth World Congress on Intelligent Transport Systems, Toronto, Ontario.

Van Der Laan, J. D., Heino, A., \& De Waard, D. (1997). A simple procedure for the assessment of acceptance of advanced transport telematics. Transportation Research Part C: Emerging Technologies, 5(1), 1-10. 
Vickrey, W. 1963. "Pricing in Urban and Suburban Transport.” American Economic Review: Papers and Proceedings 53 (2): 452-465.

Walker, J., E. Alicandri, C. Sedney, and K. Roberts. (1990). In-Vehicle Navigation Devices: Effects on the Safety of Driver Performance. Report FHWA-RD-90-053. FHWA, Washington, D.C.

Williams, C. A. (2010). A Data Mining Approach To Rapidly Learning Traveler Activity Patterns For Mobile Applications. Ph.D. Dissertation, Department of Computer Science, University of Illinois at Chicago, April 2010.

Xu Tian-dong, Yuan Hao, Zhong-ren Peng, Li-jun Sun. (2012) Real-time travel time predictor for route guidance consistent with driver behavior, Canadian Journal of Civil Engineering, 39(10): 1113-1124

Yang L. and X. Zhou. (2014). Constraint reformulation and a Lagrangian relaxation-based solution algorithm for a least expected time path problem. Transportation Research Part B: Methodological, Volume 59, Pages 22-44

Yang, L. Zhou,X. (2014). Constraint Reformulation and a Lagrangian Relaxation-based Solution Algorithm for a Least Expected Time Path Problem. Transportation Research Part B, 59, 2244

Zhang, L., and Levinson, D. (2005). An agent-based approach to travel demand forecasting: An exploratory analysis. Journal of the Transportation Research Board 1898: 28-36.

Zhang, L., Zhu, S., and Levinson, D. (2008). Agent-based modeling of price competition, capacity choice, and product differentiation in congested networks. Journal of Transport Economics and Policy 42(3): 435-461.

Zhou, X. and Taylor, J. (2014) A Queue-based mesoscopic traffic simulator for fast model evaluation and calibration. Cogent Engineering.

Zhou, X., Lu, C.-C., Zhang, K., (2012). Dynamic origin-destination demand flow estimation under congested traffic conditions: a general framework. In:(\#12-4339) The 91st Annual Meeting of Transportation Research Board, Washington, DC, USA.

Zhu, S., Levinson, D., and Zhang, L. (2008). Agent-Based Route Choice with Learning and Exchange of Information. Presented at the 87th Transportation Research Board Annual Meeting, Jan. 13 18, 2008, Washington, DC.

Ziliaskopoulos A. K. A Linear Programming Model for the Single Destination System Optimum Dynamic Traffic Assignment Problem. Transportation Science, Volume 34 Issue 1, February 2000, pp. 37-49

Ziliaskopoulos, A., \& Mahmassani, H. S. (1992). Design and implementation of a shortest path algorithm with time-dependent arc costs. In Proc. 5th Advanced Technology Conference, Washington DC (pp. 221-242). 
Ziliaskopoulos, A.K. (2000), "A linear programming model for the single destination system optimum dynamic traffic assignment problem," Transportation Science, Vol. 34, pp. 1-12. 


\section{APPEDIX A}

\section{User Experience Survey Questions}

\section{$\underline{\text { Introduction }}$}

This is a user experience survey on the usage of the virtual DMS smartphone application, VDMS, developed at the Center for Transportation Studies, the University of Virginia. You are asked to fill out the survey after using the smartphone application for several times. In this survey, questions will be asked questions about your attitude the smartphone application and preferences for some future features.

\section{$\underline{\text { Part I Basic information }}$}

Gender

O Male

Female

Age

$0<20$

○ 20-29

O 30-39

○ 40-49

○ 50-64

$O \geqslant 65$

Education level

High school or less

Some college

College graduate

Post graduate

\section{$\underline{\text { Part II Attitude Survey }}$}

This part of the questionnaire intends to capture your attitude toward the usefulness and satisfaction with the service delivered by the VDMS application. Please provide your answers to the questions below based on your experiences. The responses are going to be recorded using a five-point Likert scale (-2 to 2). Note the smartphone application refers to the app you have been using and the DMS refers to dynamic/variable message signs found along a roadway.

For After study:

what is your judgment about the VDMS system based on your experience with it in the past several weeks? Please indicate the answer based on the following eight items/attributes.

Your judgment toward the VDMS system are: (please tick a box on every line) 


\begin{tabular}{|l|l|l|l|l|l|l|l|}
\hline & & -2 & -1 & 0 & 1 & 2 & \\
\hline 1 & Useful & & & & & & Useless \\
\hline 2 & Pleasant & & & & & & Unpleasant \\
\hline 3 & Good & & & & & & Bad \\
\hline 4 & Nice & & & & & & Annoying \\
\hline 5 & Effective & & & & & & Superfluous \\
\hline 6 & Likeable & & & & & & Irritating \\
\hline 7 & Assisting & & & & & & Worthless \\
\hline 8 & Desirable & & & & & & Undesirable \\
\hline 9 & Rasing alertness & & & & & & Sleep-inducing \\
\hline
\end{tabular}

\section{Part III Experience Survey}

In this part, you will need to answer some specific questions based on your experience of VDMS system. You will also be asked questions for future app system design and development based on your stated preference.

1. Was the information provided relevant? (1 means "Completely Disagree" and 5 means "Completely Agree")
$\bigcirc 1$
$\bigcirc 2$
$\bigcirc 3$
$\bigcirc 4$
$\bigcirc$

2. Did you receive the information in time? (How was the timing?) (1 means "Really Bad" and 5 means "Really Good")
$\bigcirc 1$
$\bigcirc 2$
○3
$\bigcirc 4$
○5

3. Which message type do you prefer?

Smartphone audio message

Smartphone text message 
DMS message on a roadway

4. Is the audio message sent to your phone more comfortable to use compared with a roadside DMS text message?

$\bigcirc$ Yes $\quad \bigcirc$ No

5. Do you attach more value to (better to comply with) the audio message sent to your phone compared to a roadside DMS text message?

$\bigcirc$ Yes $\bigcirc$ No

6. What is the appropriate number of repetitions for each message? (How many times should the application read the same message to you?)
$\bigcirc 1$
$\bigcirc 2$
$\bigcirc 3$
More than 3

7. In your opinion, can the smartphone application completely substitute the current physical DMS deployments?
$\bigcirc$ Yes
$\bigcirc$ No

If not, please state the reason:

8. Please rank the following six messages that you will be willing to receive during the travel.

Occurrence and location of the incident

Expected delay (travel time)

Detour strategy

Occurrence and location of the incident + Expected delay (travel time)

Occurrence and location of the incident + the detour strategy

Expected delay (travel time) + the detour strategy

9. Would you like to receive messages at locations other than current DMS locations?

$\bigcirc$ Yes, other places on the highway where there is congestion or incidents 
$\bigcirc$ Yes, other places on the highway and arterials where there is congestion or incidents

$\bigcirc$ No

10. Under what circumstances will you opt out? (Please indicate one option)

$\bigcirc$ Too much irrelevant information

$\bigcirc$ Frequent information is noisy/distracting

$\bigcirc$ Negative impact on the battery life

Negative impact on other applications running on the phone

Other:

11. Is it acceptable if audio descriptive information (such as congestion or incident information) is provided before all major interchanges along the travel? (1 means "Definitely not acceptable"; 5 means "Very acceptable")
$\bigcirc 1$
$\bigcirc 2$
$\bigcirc 3$
$\bigcirc 4$
○ 5

12. Do you have any additional comments or suggestions about the future features, or any functional improvement to the application?

Thank you! 


\section{Appendix B}

\section{Scenarios, Experimental Messages and Maps Used in the Human Factor Study}

At the beginning of the experiment, the driver is told that he is driving along Interstate 64 Eastbound toward US 29. The alternative of I 64 for the driver is US 250, as shown in the following map In Figure B1. All the messages below apply to this driving location.

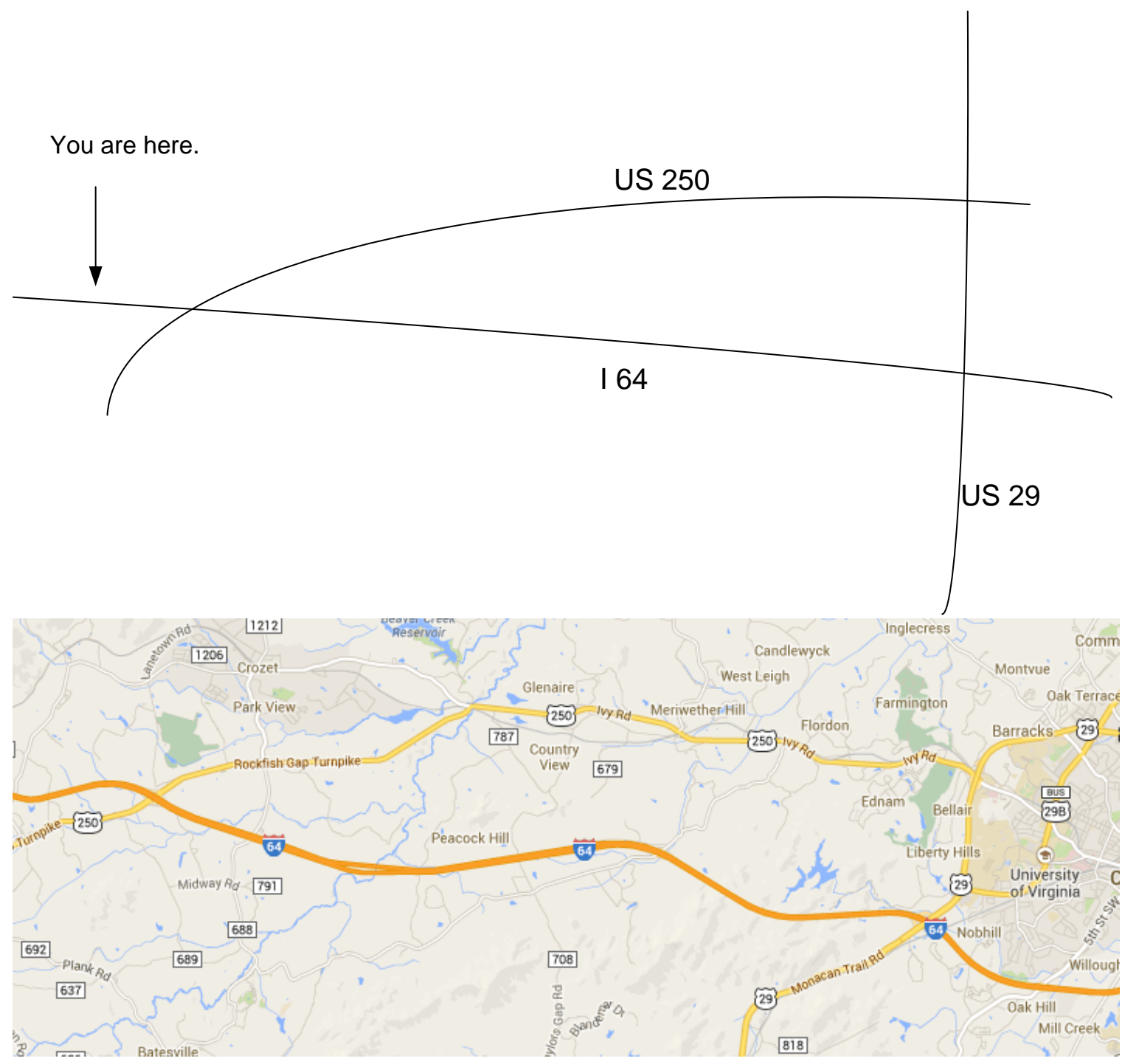

Figure B1 Maps shown to the users before the experimental design

The sixteen experimental scenarios and the numbering is shown in Table B1.

Table B1 16 Experimental scenairos 


\begin{tabular}{|l|l|l|l|}
\hline & Mode & Amount & Drive Condition \\
\hline 1 & dms & Low & Low \\
\hline 2 & vdms & Low & Low \\
\hline 3 & dms & High & Low \\
\hline 4 & dms & Low & High \\
\hline 5 & vdms & High & Low \\
\hline 6 & vdms & Low & High \\
\hline 7 & dms & High & High \\
\hline 8 & vdms & High & High \\
\hline 9 & dms & Low & Low \\
\hline 10 & vdms & Low & Low \\
\hline 11 & dms & High & Low \\
\hline 12 & dms & Low & High \\
\hline 13 & vdms & High & Low \\
\hline 14 & vdms & Low & High \\
\hline 15 & dms & High & High \\
\hline 16 & vdms & High & High \\
\hline
\end{tabular}

All the messages corresponding each of the 16 scenarios are shown below:

1.

ACCIDENT I-64 E

AT EXIT 110

\section{LEFT LANE CLOSED}

2.

New message: Roadwork at Interstate 64 Eastbound near exit 112. Right lane is closed.

3.

\begin{tabular}{|l|l|}
\hline First Phase & Second Phase \\
\hline ACCIDENT I-64 E & TIME TO US 29 \\
AT EXIT 122 & 20 MIN VIA I-64 \\
LEFT LANE CLOSED & 15 MIN VIA US-250 \\
\hline
\end{tabular}


4.

\section{EXPECT DELAY AHEAD}

\section{I-64 E TO EXIT 116}

\section{RIGHT LANE CLOSED}

5.

New message: Rock slide at Interstate 64 eastbound, near Exit 112. Right lane is closed. Expect delays, 20 minute 10 miles. Use alternative US-250.

6.

New message: Crash at Interstate 64 Eastbound near mile marker 113. Right lane is closed.

7.

\begin{tabular}{|l|l|}
\hline First Phase & Second Phase \\
\hline $\begin{array}{l}\text { ROCK SLIDE I-64 E } \\
\text { MM 109 }\end{array}$ & DELAYS \\
LEFT LANE CLOSED & 25 MIN 10 MILES \\
& USE ALT US-250 \\
\hline
\end{tabular}

8.

New Message: Major accident ahead at Interstate 64 east prior to mile marker 114, right lane is closed. Travel time to US 29 is 30 minutes via Interstate 64, and, as an alternative, 20 minutes via US 250.

9.

MOBILE ROADWORK

I-64 E EXIT 111-116

RIGHT LANE CLOSED

10. 
New Message: Delay expected at Interstate 64 eastbound after Exit 115. 20 minutes for 10 miles.

11.

\begin{tabular}{|l|l|}
\hline First Phase & Second Phase \\
\hline CRASH I-64 E & TIME TO US 29 \\
PRIOR TO EXIT 112 & 25 MIN VIA I-64 \\
EXPECT DELAY & 17 MIN VIA US-250 \\
\hline
\end{tabular}

12.

TO US 29 VIA I64

13 MILES

16 MINUTES

13.

New Message: Major accident ahead Interstate 64 East at Exit 117, right lane is closed. Heavy congestion, travel time to US 29 is 35 minutes for 10 miles. Use alternative US250.

14.

New message: Major accident ahead, left lane is closed. Use alternative US-250.

15.

\begin{tabular}{|l|l|}
\hline First Phase & Second Phase \\
\hline MAJOR CRASH I-64 E & TIME TO US-29 \\
MM 113 & 32 MIN VIA I-64 \\
EXPECT DELAY & 18 MIN VIA US-250 \\
\hline
\end{tabular}

16. 
New Message: Major accident at Interstate-64 East, 2 miles back up to exit 109. Use alternative US-250, 12 minutes for 11 miles to US 29. 